\title{
PARAMETRIZATION, STRUCTURE AND BRUHAT ORDER OF CERTAIN SPHERICAL QUOTIENTS
}

\author{
PIERRE-EMMANUEL CHAPUT, LUCAS FRESSE, AND THOMAS GOBET
}

\begin{abstract}
Let $G$ be a reductive algebraic group and let $Z$ be the stabilizer of a nilpotent element $e$ of the Lie algebra of $G$. We consider the action of $Z$ on the flag variety of $G$, and we focus on the case where this action has a finite number of orbits (i.e., $Z$ is a spherical subgroup). This holds for instance if $e$ has height 2 . In this case we give a parametrization of the $Z$-orbits and we show that each $Z$-orbit has a structure of algebraic affine bundle. In particular, in type $A$, we deduce that each orbit has a natural cell decomposition. In the aim to study the (strong) Bruhat order of the orbits, we define an abstract partial order on certain quotients associated to a Coxeter system. In type $A$, we show that the Bruhat order of the $Z$-orbits can be described in this way.
\end{abstract}

\section{Contents}

Introduction

Part 1. Structure of the isotropy group of a spherical nilpotent orbit

1. Parabolic subgroup associated to a nilpotent element

2. Relation between normalizer and stabilizer

3. Nilpotent orbits and orthogonal roots

4. Symmetric subgroup associated to a spherical nilpotent orbit 950

5. Focus on nilpotent elements of height 2

Part 2. Structure of orbits on the flag variety for the action of certain spherical subgroups obtained through parabolic induction

6. Notation and short review on parabolic orbits

7. Parametrization and structure of $H$-orbits

Part 3. Bruhat order

8. A Coxeter-theoretic partial order $\quad 959$

9. Inclusions of orbit closures in type $A$

References

Received by the editors September 18, 2020, and, in revised form, June 2, 2021.

2020 Mathematics Subject Classification. Primary 20G05, 17 B08.

The first two authors were supported in part by the ANR project GeoLie ANR-15-CE400012. The third author was partially supported by the same project and by the ARC project DP170101579. 


\section{INTRODUCTION}

Let $G$ be a connected reductive algebraic group over $\mathbb{K}$, where $\mathbb{K}$ denotes an algebraically closed field of characteristic zero. Let $B \subset G$ be a Borel subgroup. A closed subgroup $H \subset G$ is said to be spherical if the homogeneous space $G / H$ has a finite number of $B$-orbits, equivalently if the flag variety $\mathcal{B}:=G / B$ has a finite number of $H$-orbits.

Let $\mathfrak{g}$ be the Lie algebra of $G$. Let $e \in \mathfrak{g}$ be a nilpotent element. The following subgroups can be attached to $e$ : the stabilizer

$$
Z_{G}(e):=\{g \in G: g \cdot e=e\}
$$

and the normalizer

$$
N_{G}(e):=\{g \in G: g \cdot(\mathbb{K} e)=\mathbb{K} e\} .
$$

Our concern is the action of $B$ on the nilpotent orbit $G \cdot e=G / Z_{G}(e)$ or equivalently the action of $Z_{G}(e)$ on $G / B$. We focus on the case where this action comprises a finite number of orbits, which means that the subgroup $Z_{G}(e)$ is spherical. In this situation, our motivation is to understand

(1) the parametrization and the structure of the $Z_{G}(e)$-orbits on $\mathcal{B}$;

(2) the inclusion relations between the orbit closures.

Beyond the fact that the $Z_{G}(e)$-orbits give an interesting partition of the whole flag variety, the action of $Z_{G}(e)$ restricts to certain subvarieties of $\mathcal{B}$ (Springer varieties, Hessenberg varieties) which arise in geometric representation theory. Information on the structure and the topology of the orbits may have applications in this direction.

The nilpotent elements $e$ such that $Z_{G}(e)$ is spherical are classified by Panyushev Pa94, Pa99 as those of height at most 3. The problem of classifying the $Z_{G}(e)$-orbits of $G / B$ has been considered in certain (mostly, classical) cases in BP19, BCE19, BR12]. The problem of describing the inclusion relations between the orbit closures has been addressed in BP19, BR12] in certain cases. To the best of our knowledge, there is no general approach to these problems, although recently GMP20] associate to any nilpotent element of height 2 an involution in the affine Weyl group, and show that the orbit closures are described by restricting the Bruhat order on the affine Weyl group.

The paper is divided into three parts. In Part 1, we review the general background on nilpotent elements and nilpotent orbits, including Panyushev's classification of spherical nilpotent orbits. Then we focus on the structure of the group $Z_{G}(e)$ and point out the following facts. In general, $Z_{G}(e)$ has a "Levi decomposition" of the form

$$
Z_{G}(e)=L_{Z} \ltimes U_{Z}
$$

with reductive part $L_{Z} \subset L$ and unipotent part $U_{Z} \subset U$, respectively contained in the Levi subgroup and the unipotent radical of a suitable parabolic subgroup $P=L \ltimes U$ attached to $e$. In the case where $Z_{G}(e)$ is spherical, it turns out that the reductive part $L_{Z}$ coincides with the subgroup of fixed points of an involution $\sigma \in \operatorname{Aut}(L)$ (possibly up to connected components). This follows from Panyushev's classification but we provide a direct argument, defining the involution as the nontrivial element of the Weyl group of an $\mathrm{SL}_{2}$-subgroup associated with the nilpotent element $e$ (see Proposition 4.5). Finally, in the more particular situation where $e$ is a nilpotent element of height 2 , the unipotent part $U_{Z}$ coincides with the full 
unipotent radical $U$. Hence $Z_{G}(e)$ is obtained through parabolic induction from a symmetric subgroup of $L$ in this case.

One of the main ingredients used for Proposition 4.5 is a classification of spherical nilpotent orbits in terms of sets of so-called rationally orthogonal roots (see Proposition [3.3), which extends Panyushev's classification in terms of orthogonal simple roots. The notion of rationally orthogonal roots differs from those of orthogonal or strongly orthogonal sets of roots, and this difference is thoroughly discussed in Section 3 ,

Another aspect considered in Part 1 is the comparison between the $Z_{G}(e)$-orbits and $N_{G}(e)$-orbits of $\mathcal{B}$. We show that under certain circumstances (including the case where $Z_{G}(e)$ is spherical), both sets of orbits actually coincide (Proposition 2.3). We however point out an example which shows that the subgroup $N_{G}(e)$ may be spherical whereas $Z_{G}(e)$ is not. We believe that the comparison of these two sets of orbits may be a problem of independent interest.

In Part 2, we focus on a spherical subgroup of the form

$$
H=M \ltimes U,
$$

where $P=L \ltimes U$ is the Levi decomposition of a parabolic subgroup and $M \subset L$ is a spherical subgroup. In particular, the role of $H$ can be played by the stabilizer $Z_{G}(e)$ of a nilpotent element of height 2 . For $H$ as above, we show that the $H$-orbits of $\mathcal{B}$ are naturally parametrized by the set

$$
W^{P} \times\left(\mathcal{B}_{L} / M\right),
$$

where $W^{P}$ is the Weyl group parabolic quotient associated to $P$ (i.e., the set of representatives of minimal length of the quotient $\left.W / W_{P}\right)$ and $\mathcal{B}_{L}$ is the flag variety of $L$. Moreover, in our main result (Theorem 7.2 ) we prove that each orbit has a structure of algebraic affine bundle over an $M$-orbit of $\mathcal{B}_{L}$. In type $A$, we deduce that each $Z_{G}(e)$-orbit has a natural cell decomposition (Example 7.3).

In Part 3, we focus on the (strong) Bruhat order of the $Z_{G}(e)$-orbits. We introduce a combinatorial order which reflects the geometric situation described above. Specifically, given a Coxeter system $(W, S)$, we consider a parabolic subgroup $W_{L} \subset W$ equipped with an involution $\theta: W_{L} \rightarrow W_{L}$. Then, we introduce a partial order on the quotient $W / W_{L}^{\theta}$, where $W_{L}^{\theta}$ stands for the subgroup of fixed points of $\theta$. We mostly address the situation where $W_{L}^{\theta}$ is a diagonal subgroup of $W_{L}$. We investigate certain properties of this order (minimal representatives, cover relations).

In type $A_{n-1}$, for a nilpotent element $e$ of height 2 , the $Z_{G}(e)$-orbits of the flag variety $\mathcal{B}$ are parametrized by a quotient of the above-mentioned form, namely $\mathfrak{S}_{n} /\left(\Delta \mathfrak{S}_{r} \times \mathfrak{S}_{n-2 r}\right)$, where $\Delta \mathfrak{S}_{r}$ stands for the diagonal embedding of $\mathfrak{S}_{r}$ into $\mathfrak{S}_{r} \times \mathfrak{S}_{r}$. Then, translating the results of [BP19, BR12] into our framework, we show that our combinatorial order coincides with the Bruhat order of the $Z_{G}(e)$ orbits.

\section{Part 1. Structure of the isotropy group of a spherical nilpotent orbit}

Throughout this part of the paper, we fix a nilpotent element $e \in \mathfrak{g}$ and our aim is to describe the structure of its stabilizer

$$
Z:=Z_{G}(e)=\{g \in G: \operatorname{Ad}(g) e=e\} .
$$


We are mostly concerned with the case where the corresponding orbit $\mathcal{O}_{e}:=G \cdot e$ is a spherical variety, i.e., it consists of a finite number of $B$-orbits. Equivalently this means that $Z$ is a spherical subgroup, i.e., the flag variety $\mathcal{B}$ has a finite number of $Z$-orbits.

In Section 11 we introduce the basic ingredients which are useful for describing the structure of $Z$ : namely, we recall the notions of standard triple, cocharacter $\tau$ and parabolic subgroup $P$ associated to $e$. In particular we recall that the stabilizer has a "Levi decomposition"

$$
Z=L_{Z} \ltimes U_{Z} .
$$

In general the subgroup $L_{Z}$ is not connected. Note that in general the group $Z$ may not be connected even in the case where the orbit $\mathcal{O}_{e}$ is spherical.

The nilpotent orbits which are spherical are classified in $[\mathrm{Pa94}, \mathrm{Pa99}$ and this classification is recalled in Proposition [3.1. In particular, one characterization is that every spherical nilpotent orbit has a representative obtained as sum of root vectors corresponding to a set of pairwise orthogonal simple roots; see Proposition 3.1(iii). In Proposition 3.3. we provide a more general classification in terms of sets of so-called rationally orthogonal (not necessarily simple) roots, which is the form that we will need in Proposition 4.5 to show that a spherical nilpotent orbit defines a symmetric space. In Proposition 3.7. we give a somewhat more precise result where we characterize the sets of orthogonal roots (not necessarily simple nor rationally orthogonal) for which a sum of root vectors belongs to a spherical nilpotent orbit. In Example 3.15 we also point out that in various cases, the sets of orthogonal roots corresponding to spherical nilpotent orbits can be obtained by chain cascade of roots.

In the case where the orbit $\mathcal{O}_{e}$ is spherical, we obtain the following description of the group $Z$. In Section 4 we show that the "Levi subgroup" $L_{Z}$ of $Z$ is a symmetric subgroup of a Levi subgroup $L \subset P$ (possibly up to certain connected components). This fact is already known from [Pa94, Proposition 3.3] (at the level of the Lie algebras), but our proof is somewhat different. In particular we give an explicit construction of an involution $\sigma \in \operatorname{Aut}(L)$ such that $L_{Z}$ coincides with the subgroup $L^{\sigma}$ of fixed points of $\sigma$ (up to connected components of $L^{\sigma}$ ).

In Section 5. considering the special case where $e$ is a nilpotent element of height 2, we point out that $Z$ contains the unipotent radical of $P$. Thus $Z$ is obtained by parabolic induction from a symmetric subgroup of a Levi factor of $P$.

We believe that Section 2 is of independent interest. In that section, we compare the $Z$-orbits on $\mathcal{B}$ with the orbits of the normalizer $N:=N_{G}(e)=\{g \in G$ : $\operatorname{Ad}(g) e \in \mathbb{K} e\}$. In particular we show that in the case where $\mathcal{O}_{e}$ is spherical, both sets of orbits coincide, whereas this is not the case in general.

\section{Parabolic subgroup associated to A nilpotent Element}

By the Jacobson-Morozov lemma, every nilpotent element $e \in \mathfrak{g}$ is member of a standard triple, i.e., there exist $h, f \in \mathfrak{g}$ such that

$$
[h, e]=2 e, \quad[h, f]=-2 f, \quad[e, f]=h,
$$

so that $\operatorname{Span}\{e, h, f\} \subset \mathfrak{g}$ is a subalgebra isomorphic to $\mathfrak{s l}_{2}(\mathbb{K})$.

The semisimple element $h$ gives rise to a $\mathbb{Z}$-grading

$$
\mathfrak{g}=\bigoplus_{i \in \mathbb{Z}} \mathfrak{g}(i) \quad \text { where } \quad \mathfrak{g}(i)=\{x \in \mathfrak{g}:[h, x]=i x\} .
$$


The nonnegative part of the grading $\mathfrak{p}:=\bigoplus_{i>0} \mathfrak{g}(i)$ is a parabolic subalgebra, the zero part of the grading $\mathfrak{l}:=\mathfrak{g}(0)=\mathfrak{z} \mathfrak{g}(h)$ is a Levi subalgebra of $\mathfrak{p}$, and the positive part of the grading $\mathfrak{u}:=\bigoplus_{i>0} \mathfrak{g}(i)$ is the nilpotent radical of $\mathfrak{p}$. Correspondingly the grading yields a parabolic subgroup $P$ and a Levi decomposition

$$
P=L \ltimes U
$$

such that $\mathfrak{p}=\operatorname{Lie}(P), \mathfrak{l}=\operatorname{Lie}(L)$, and $\mathfrak{u}=\operatorname{Lie}(U)$.

By the representation theory of $\mathfrak{s l}_{2}(\mathbb{K})$, we have the inclusion

$$
\mathfrak{z} \mathfrak{g}(e)=\operatorname{Lie}(Z) \subset \bigoplus_{i \geq 0} \mathfrak{g}(i)=\mathfrak{p}
$$

and the dimension formula

$$
\operatorname{dim} \mathfrak{z}_{\mathfrak{g}}(e)=\operatorname{dim} \mathfrak{g}(0)+\operatorname{dim} \mathfrak{g}(1) .
$$

Moreover, there is a (unique) cocharacter $\tau: \mathbb{K}^{*} \rightarrow G$ such that $\tau^{\prime}(1)=h$. This implies that the parabolic subgroup $P \subset G$ and its Levi decomposition $P=L \ltimes U$ can also be characterized as follows:

$$
\begin{gathered}
P=\left\{g \in G: \lim _{t \rightarrow 0} \tau(t) g \tau(t)^{-1} \text { exists }\right\}, \\
L=\left\{g \in G: \forall t \in \mathbb{K}^{*}, \tau(t) g \tau(t)^{-1}=g\right\}=Z_{G}(\tau), \\
U=\left\{g \in G: \lim _{t \rightarrow 0} \tau(t) g \tau(t)^{-1}=1_{G}\right\} .
\end{gathered}
$$

Proposition 1.1.

(a) We have $Z \subset P$. Letting $U_{Z}:=U \cap Z$ and $L_{Z}:=L \cap Z$, we have

$$
Z=L_{Z} \ltimes U_{Z} \text {. }
$$

Moreover, the subgroup $U_{Z}$ is connected.

(b) Considering the connected subgroup $Z^{0} \subset Z$, we have $L \cap\left(Z^{0}\right)=\left(L_{Z}\right)^{0}=$ : $L_{Z}^{0}, U \cap\left(Z^{0}\right)=U_{Z}$, and

$$
Z^{0}=L_{Z}^{0} \ltimes U_{Z} .
$$

(c) If $S$ is a maximal torus of $L_{Z}^{0}$, then $S$ is a maximal torus of $Z$.

Proof. (a) Let $g \in Z$. Then $(e, g \cdot h, g \cdot f)$ is a standard triple which also contains the element $e$. By Kostant's theorem [CMcG93, Theorem 3.4.10], there is an element $u \in U \cap Z$ such that $g \cdot h=u \cdot h$ and $g \cdot f=u \cdot f$. In particular $u^{-1} g \cdot h=h$, which means that $u^{-1} g=: \ell \in L \cap Z$. Whence $g=u \ell \in P$. This argument shows in fact that the inclusion $Z \subset L_{Z} U_{Z}$ holds. Since $L_{Z} \cap U_{Z}=\left\{1_{G}\right\}$, we conclude that $Z=L_{Z} \ltimes U_{Z}$.

Letting $u \in U_{Z}$, we have for all $t \in \mathbb{K}^{*}$

$$
\operatorname{Ad}\left(\tau(t) u \tau(t)^{-1}\right) e=t^{-2} \operatorname{Ad}(\tau(t) u) e=t^{-2} \operatorname{Ad}(\tau(t)) e=e,
$$

hence $\left\{\tau(t) u \tau(t)^{-1}: t \in \mathbb{K}^{*}\right\} \subset U_{Z}$. Since $\lim _{t \rightarrow 0} \tau(t) u \tau(t)^{-1}=1_{G}$, we conclude that $u \in\left(U_{Z}\right)^{0}$. This shows that $U_{Z}$ is connected.

(b) Since $U_{Z}$ is connected, we have $U_{Z} \subset Z^{0}$, hence $U_{Z}=U \cap\left(Z^{0}\right)$. By (a), we get the equality $Z^{0}=\left(L \cap\left(Z^{0}\right)\right) \ltimes U_{Z}$, which also implies that $L \cap\left(Z^{0}\right)$ is connected, i.e., $L \cap\left(Z^{0}\right)=\left(L_{Z}\right)^{0}$.

(c) Let $S$ be a maximal torus of $L_{Z}^{0}$ and let $T$ be a maximal torus of $Z$ such that $S \subset T$. Thus $T \subset Z^{0}$. In view of (b), there is a surjective morphism of algebraic groups $\pi: Z^{0} \rightarrow L_{Z}^{0}=Z^{0} / U_{Z}$. Since $S$ is a maximal torus of $L_{Z}^{0}$, we deduce that 
$\pi(T)=S$. On the other hand, since every element of $U_{Z}$ is unipotent, $T \cap U_{Z}$ must be trivial. Therefore, the equality $S=T$ must hold.

\section{RELATION BETWEEN NORMALIZER AND STABILIZER}

The normalizer of $e \in \mathfrak{g}$ is the subgroup

$$
N:=N_{G}(e)=\{g \in G: \operatorname{Ad}(g) e \in \mathbb{K} e\} .
$$

We have the following relation between $Z$ and $N$; here $\tau$ is the cocharacter associated to $e$ as in Section 1 .

Proposition 2.1. $Z$ is a normal subgroup of $N$. We have $N=Z\{\tau(t)\}_{t \in \mathbb{K}^{*}}$ and $Z \cap\{\tau(t)\}_{t \in \mathbb{K}^{*}}$ contains at most two elements.

Proof. The first claim is clear. For $g \in N$, we have $\operatorname{Ad}(g) e=t^{2} e$ for some $t \in$ $\mathbb{K}^{*}$, hence $\operatorname{Ad}\left(g^{-1} \tau(t)\right) e=e$, i.e., $g \in Z \tau(t)$. Finally, for $t \in \mathbb{K}^{*}$, the equality $\operatorname{Ad}(\tau(t)) e=t^{2} e$ implies that $\tau(t)$ belongs to $Z$ if and only if $t \in\{-1,1\}$.

Remark 2.2. If the nilpotent element $e$ is even (that is, if the grading of Section 1 satisfies $\mathfrak{g}(i)=0$ for all odd $i)$, then $\tau(-1)=\tau(1)=1_{G}$. In this case, we have $Z \cap\{\tau(t)\}_{t \in \mathbb{K}^{*}}=\left\{1_{G}\right\}$, so that $N=Z \rtimes\{\tau(t)\}_{t \in \mathbb{K}^{*}}$.

We now compare the actions of $N$ and $Z$ on the flag variety $\mathcal{B}$. Evidently, each $Z$-orbit is contained in an $N$-orbit, and this implies that $N$ is a spherical subgroup whenever $Z$ is a spherical subgroup. There are situations where the $N$-orbits and the $Z$-orbits of $\mathcal{B}$ actually coincide.

\section{Proposition 2.3.}

(a) If every $N$-orbit (or every $Z$-orbit) of $\mathcal{B}$ contains an element fixed by $\tau$, then the $Z$-orbits of $\mathcal{B}$ coincide with the $N$-orbits.

(b) If $Z$ is a spherical subgroup of $G$, then the $Z$-orbits of $\mathcal{B}$ coincide with the $N$-orbits.

Proof. (a) Assume that every $N$-orbit of $\mathcal{B}$ contains an element fixed by $\tau$ (this is more general than assuming that every $Z$-orbit contains such an element). Then every $N$-orbit takes the form $N \cdot g B$ with $\tau(t) g B=g B$ for all $t \in \mathbb{K}^{*}$. In view of Proposition 2.1, we get $N \cdot g B=Z \cdot g B$, which shows that every $N$-orbit is actually a $Z$-orbit.

(b) Assume that $Z$ is a spherical subgroup. Let $\mathcal{N}:=N \cdot g B$ be an $N$-orbit of $\mathcal{B}$; in particular $\mathcal{N}$ contains finitely many $Z$-orbits. We consider the map

$$
\phi: \mathbb{K}^{*} \rightarrow \mathcal{N}, t \mapsto \phi(t):=\tau(t) g B
$$

Note that every $Z$-orbit of $\mathcal{N}$ is of the form $Z \cdot \tau(t) g B$ for some $t \in \mathbb{K}^{*}$. We have

$$
\begin{aligned}
\operatorname{dim} Z \cdot \tau(t) g B & =\operatorname{dim} Z-\operatorname{dim}\{h \in Z: h \tau(t) g B=\tau(t) g B\} \\
& =\operatorname{dim} Z-\operatorname{dim} \tau(t)^{-1} Z \tau(t) \cap g B g^{-1} \\
& =\operatorname{dim} Z-\operatorname{dim} Z \cap g B g^{-1}
\end{aligned}
$$

because $\tau(t)^{-1} Z \tau(t)=Z$. This shows that all the $Z$-orbits contained in $\mathcal{N}$ have the same dimension. In particular this implies that

$$
\mathcal{Z}=\overline{\mathcal{Z}} \cap \mathcal{N} \quad \text { for every } Z \text {-orbit } \mathcal{Z} \subset \mathcal{N} \text {. }
$$

Since the map $\phi$ is algebraic, we obtain

$$
\left\{s \in \mathbb{K}^{*}: \phi(s) \in \mathcal{Z}\right\} \text { is closed in } \mathbb{K}^{*} \text { for every } Z \text {-orbit } \mathcal{Z} \subset \mathcal{N} .
$$


Since $\mathcal{N}$ contains a finite number of $Z$-orbits, there is a $Z$-orbit $\mathcal{Z} \subset \mathcal{N}$ such that $\left\{s \in \mathbb{K}^{*}: \phi(s) \in \mathcal{Z}\right\}=\mathbb{K}^{*}$, which just means that $\mathcal{Z}=\mathcal{N}$.

Remark 2.4.

(a) In general, $Z$-orbits and $N$-orbits of $\mathcal{B}$ do not coincide. It actually may happen that $N$ is spherical whereas $Z$ is not, as shown by the following example. Let us consider the situation where $\mathfrak{g}=\mathfrak{s l}_{3}(\mathbb{K})$ and

$$
e=\left(\begin{array}{lll}
0 & 1 & 0 \\
0 & 0 & 1 \\
0 & 0 & 0
\end{array}\right) \text {. }
$$

In this case

$$
\begin{gathered}
Z=\left\{\left(\begin{array}{ccc}
a & b & c \\
0 & a & b \\
0 & 0 & a
\end{array}\right): a \in \mathbb{K}^{*} \text { s.t. } a^{3}=1 ; b, c \in \mathbb{K}\right\}, \\
N=\{\tau(t)\}_{t \in \mathbb{K}^{*}} Z \quad \text { with } \quad \tau(t)=\left(\begin{array}{ccc}
t^{2} & 0 & 0 \\
0 & 1 & 0 \\
0 & 0 & t^{-2}
\end{array}\right) .
\end{gathered}
$$

Let $\left(\varepsilon_{1}, \varepsilon_{2}, \varepsilon_{3}\right)$ be the standard basis of $\mathbb{K}^{3}$. Here the flag variety $\mathcal{B}$ can be viewed as the set of complete flags of $\mathbb{K}^{3}$. Such a complete flag consists of a pair $\left(F_{1}, F_{2}\right)$ with $\operatorname{dim} F_{1}=1, \operatorname{dim} F_{2}=2,0 \subset F_{1} \subset F_{2} \subset \mathbb{K}^{3}$. Given linearly independent vectors $v_{1}, v_{2}$, we write $F\left(v_{1}, v_{2}\right):=\left(\left\langle v_{1}\right\rangle,\left\langle v_{1}, v_{2}\right\rangle\right)$. It is easy to see that the elements $F\left(\varepsilon_{3}, \varepsilon_{1}+t \varepsilon_{2}\right)$, for $t \in \mathbb{K}$, belong to pairwise distinct $Z$-orbits of $\mathcal{B}$. Thus $\mathcal{B}$ has infinitely many $Z$-orbits. However, $\mathcal{B}$ has exactly seven $N$-orbits, whose representatives are

$$
F\left(\varepsilon_{1}, \varepsilon_{2}\right), F\left(\varepsilon_{1}, \varepsilon_{3}\right), F\left(\varepsilon_{2}, \varepsilon_{1}\right), F\left(\varepsilon_{2}, \varepsilon_{3}\right), F\left(\varepsilon_{3}, \varepsilon_{1}\right), F\left(\varepsilon_{3}, \varepsilon_{2}\right), F\left(\varepsilon_{3}, \varepsilon_{1}+\varepsilon_{2}\right) .
$$

Only the last one of these orbits does not contain any element fixed by $\tau$.

(b) In Remark 5.3(b) we point out an example where $Z$ is spherical (thus $Z$ orbits coincide with $N$-orbits by Proposition $2.3(\mathrm{~b}))$ though there is a $Z$-orbit of $\mathcal{B}$ which contains no point fixed by $\tau$. Hence the converse of Proposition 2.3(a) is in general not true.

\section{Nilpotent orbits AND ORThOGOnAl RoOts}

Before stating a list of necessary and sufficient conditions for $Z=Z_{G}(e)$ to be a spherical subgroup of $G$, we recall some notions related to nilpotent elements.

Since $e$ is a nilpotent element, its image by the adjoint representation is a nilpotent endomorphism ad $e: \mathfrak{g} \rightarrow \mathfrak{g}$. Then, the height of $e$ is defined as the biggest integer $k \geq 0$ such that $(\operatorname{ad} e)^{k} \neq 0$. Equivalently, $k$ is maximal such that $\mathfrak{g}(k) \neq 0$, for the grading $\mathfrak{g}=\bigoplus_{i \in \mathbb{Z}} \mathfrak{g}(i)$ of Section 1 ,

If we take a maximal torus $S$ of $Z$, then the Lie algebra

$$
\mathfrak{g}_{0}(e):=\operatorname{Lie}\left(Z_{G}(S)\right)=\{x \in \mathfrak{g}: \forall s \in S, \operatorname{Ad}(s) x=x\}
$$

is a Levi subalgebra of $\mathfrak{g}$ which contains $e$ and which is minimal for this property. The Lie algebra $\mathfrak{g}_{0}(e)$ does not essentially depend on the choice of the torus $S$. The type of the semisimple Lie algebra $\left[\mathfrak{g}_{0}(e), \mathfrak{g}_{0}(e)\right]$ is referred to as the type of the nilpotent orbit $G \cdot e$. This datum arises in the classification of nilpotent orbits due to Bala and Carter; see [CMcG93, §8] for more details. 
In the next statement, we also fix a root space decomposition $\mathfrak{g}=\mathfrak{t} \oplus \bigoplus_{\alpha \in \Phi} \mathfrak{g}_{\alpha}$ and a system of positive roots $\Phi^{+} \subset \Phi$. Every nilpotent element of $\mathfrak{g}$ lies in the (adjoint) $G$-orbit of an element of the space $\bigoplus_{\alpha \in \Phi^{+}} \mathfrak{g}_{\alpha}$. There is no loss of generality in assuming that the image of the cocharacter $\tau$ associated to $e$ is contained in the maximal torus $T \subset G$ such that $\mathfrak{t}=\operatorname{Lie}(T)$.

Proposition 3.1 ([Pa9, $\mathrm{Pa99}])$. The following conditions are equivalent:

(i) $Z=Z_{G}($ e) is a spherical subgroup of $G$;

(ii) The height of $e$ is at most 3;

(iii) e belongs to the $G$-orbit of an element obtained as the sum of root vectors corresponding to pairwise orthogonal simple roots;

(iv) Every simple factor of $\left[\mathfrak{g}_{0}(e), \mathfrak{g}_{0}(e)\right]$ is of type $A_{1}$.

Note that the condition in Proposition 3.1(iii) (which corresponds to Pa99, Theorem 3.4]) yields a kind of normal form for spherical nilpotent orbits. We actually need a slightly different version of Proposition 3.1(iii), which we give in the next statement.

Recall that two roots $\alpha, \beta \in \Phi$ are said to be strongly orthogonal if $\alpha+\beta$ and $\alpha-\beta$ do not belong to $\Phi \cup\{0\}$. In particular, this implies that $\alpha$ and $\beta$ are orthogonal. We will need a somewhat more restrictive condition that we now state:

Definition 3.2. Let $\beta_{1}, \ldots, \beta_{k} \in \Phi$ be linearly independent. We say that $\beta_{1}, \ldots, \beta_{k}$ are rationally orthogonal if

$$
\left(\bigoplus_{i=1}^{k} \mathbb{Q} \beta_{i}\right) \cap \Phi=\left\{ \pm \beta_{1}, \pm \beta_{2}, \ldots, \pm \beta_{k}\right\} .
$$

A mistake in a previous version of this paper occurring in Proposition 3.3 has been pointed out to us by J. Gandini, to whom we are grateful.

\section{Proposition 3.3.}

(a) Let $\theta_{1}, \ldots, \theta_{r} \in \Phi$ be a sequence of rationally orthogonal roots (not necessarily simple nor positive). For $i \in\{1, \ldots, r\}$, let $e_{\theta_{i}} \in \mathfrak{g}_{\theta_{i}} \backslash\{0\}$. Then $e=\sum_{i} e_{\theta_{i}}$ belongs to a spherical nilpotent orbit of type $r A_{1}$.

(b) Conversely, every spherical nilpotent orbit contains an element of the form $e=\sum_{i} e_{\theta_{i}}$ corresponding to a sequence $\theta_{1}, \ldots, \theta_{r}$ of rationally orthogonal roots, where we may assume in addition that $\sum_{i} \theta_{i}^{\vee}$ is a dominant coweight.

Proof. (a) The element $e=\sum_{i} e_{\theta_{i}}$ is nilpotent because, knowing that $\theta_{1}, \ldots, \theta_{r}$ are linearly independent, there can be only finitely many pairs $\left(\alpha,\left(n_{i}\right)\right)$ where $\alpha$ is 0 or a root and $\left(n_{i}\right)=\left(n_{1}, \ldots, n_{r}\right)$ is a sequence of integers, such that $\alpha+\sum_{i} n_{i} \theta_{i}$ is 0 or a root. Note that $h:=\sum_{i} \theta_{i}^{\vee}$ (as an element of $\mathfrak{t}$ ) satisfies $[h, e]=2 e$, and we can find $f_{\theta_{i}} \in \mathfrak{g}_{-\theta_{i}}$ such that $\left(e, h, \sum_{i} f_{\theta_{i}}\right)$ is a standard triple. This implies that $\tau:=\sum_{i} \theta_{i}^{\vee}$ (seen this time as a cocharacter of $T$ ) is a cocharacter associated to $e$ in the sense of Section 1

If $\alpha$ is a root, we denote $\mathfrak{s l}_{2}(\alpha):=\mathfrak{g}_{-\alpha} \oplus\left[\mathfrak{g}_{-\alpha}, \mathfrak{g}_{\alpha}\right] \oplus \mathfrak{g}_{\alpha}$, which is a subalgebra of $\mathfrak{g}$ isomorphic to $\mathfrak{s l}_{2}(\mathbb{K})$.

Note that $S:=\bigcap_{i} \operatorname{ker} \theta_{i}$ is a torus contained in $Z$; actually it is also contained in the subgroup $L_{Z}^{0}=Z_{G}(\tau) \cap Z^{0}$. In view of Proposition 1.1, there is a maximal torus $S^{\prime}$ of $Z$ such that $S \subset S^{\prime} \subset L_{Z}^{0}$.

We claim that the centralizer of $S \cdot \tau\left(\mathbb{K}^{*}\right)$ is $T$. Indeed, for otherwise, there is a root $\alpha$ which is trivial on $S \cdot \tau\left(\mathbb{K}^{*}\right)$. Since $\alpha$ is in particular trivial on $S$, it is a 
linear combination of the roots $\theta_{i}$ with rational coefficients. Since the roots $\theta_{i}$ are rationally orthogonal, there is an integer $i$ such that $\alpha= \pm \theta_{i}$. Finally, since $\alpha$ must also be trivial on $\tau=\theta_{1}^{\vee}+\cdots+\theta_{r}^{\vee}$, we get a contradiction. Thus, the centralizer of $S \cdot \tau\left(\mathbb{K}^{*}\right)$ is $T$. This implies that $S^{\prime} \subset T$, hence $\mathfrak{t} \subset \mathfrak{z}_{\mathfrak{g}}\left(S^{\prime}\right):=\{x \in \mathfrak{g}: \forall s \in$ $\left.S^{\prime}, \operatorname{Ad}(s) x=x\right\}$.

Our argument also shows that

$$
\mathfrak{z}_{\mathfrak{g}}(S)=\mathfrak{t}+\bigoplus_{i=1}^{r} \mathfrak{s l}_{2}\left(\theta_{i}\right) \quad \text { and so } \quad\left[\mathfrak{z}_{\mathfrak{g}}(S), \mathfrak{z}_{\mathfrak{g}}(S)\right]=\bigoplus_{i=1}^{r} \mathfrak{s l}_{2}\left(\theta_{i}\right) .
$$

Since $\mathfrak{t}+\mathbb{K} e \subset \mathfrak{z}_{\mathfrak{g}}\left(S^{\prime}\right) \subset \mathfrak{z}_{\mathfrak{g}}(S)$, we get $\mathfrak{z}_{\mathfrak{g}}\left(S^{\prime}\right)=\mathfrak{z}_{\mathfrak{g}}(S)$, i.e., $\mathfrak{g}_{0}(e)=\mathfrak{z}_{\mathfrak{g}}(S)$ with the notation of Proposition [3.1. Hence $\left[\mathfrak{g}_{0}(e), \mathfrak{g}_{0}(e)\right]$ is of type $r A_{1}$ and, since the condition in Proposition 3.1(iv) is satisfied, the orbit $G \cdot e$ is spherical.

(b) Conversely, let $e$ be a nilpotent element and $S$ a maximal torus of $Z$ contained in $L_{Z}^{0}$. Up to the action of an element of $G$, we may assume that $S \cdot \tau\left(\mathbb{K}^{*}\right) \subset T$. Assuming that $e$ is spherical, by Proposition 3.1(iii), we may write $e=\sum_{i} e_{\theta_{i}}$ with $\left(\theta_{1}, \ldots, \theta_{r}\right)$ a sequence of rationally orthogonal roots.

If the sum $\sum_{i} \theta_{i}^{\vee}$ is not dominant, then there is a simple root $\alpha$ such that $s_{\alpha}\left(\sum_{i} \theta_{i}^{\vee}\right)>\sum_{i} \theta_{i}^{\vee}$. Then, $\left(s_{\alpha}\left(\theta_{1}\right), \ldots, s_{\alpha}\left(\theta_{r}\right)\right)$ is again a sequence of rationally orthogonal roots with $\sum_{i} s_{\alpha}\left(\theta_{i}\right)^{\vee}>\sum_{i} \theta_{i}^{\vee}$. The $W$-orbit of $\sum_{i} \theta_{i}^{\vee}$ being bounded, this process must terminate with some sequence $\left(\theta_{i}\right)$ such that the sum $\sum_{i} \theta_{i}^{\vee}$ is dominant.

Remark 3.4. For $\left(\theta_{i}\right)$ such that $\sum_{i} \theta_{i}^{\vee}$ is dominant, if we label the vertex $\alpha$ of the Dynkin diagram of $\mathfrak{g}$ with the number $\alpha\left(\sum_{i} \theta_{i}^{\vee}\right)$, then we obtain a weighted Dynkin diagram which is precisely the one parametrizing the nilpotent orbit $\mathcal{O}_{e}$ in the sense of [CMcG93, §3.5]. In particular the coweight $\sum_{i} \theta_{i}^{\vee}$ is independent of the sequence $\left(\theta_{i}\right)$ involved in the statement.

We now give a quite precise description of when rational orthogonality differs from orthogonality. We write $\Phi \supset X_{r}$ to mean that the root system of $\Phi$ contains a root subsystem of type $X_{r}$.

Lemma 3.5. Let $\beta_{1}, \ldots, \beta_{k}$ be a set of orthogonal roots and let $n_{1}, \ldots, n_{k} \in \mathbb{Q} \backslash\{0\}$ be such that $\sum n_{i} \beta_{i} \in \Phi$. Then one of the following holds:

$\left(D_{4}\right) k=4$, all the roots $\beta_{i}$ have the same length, $\frac{1}{2}\left(\beta_{1}+\beta_{2}+\beta_{3}+\beta_{4}\right)$ is a root, and $\Phi \supset D_{4}$.

$\left(B_{3}\right) k=3$ and, up to reordering the roots $\beta_{i}$, we have that $\beta_{1}$ and $\beta_{2}$ are long and $\beta_{3}$ is short, $\frac{1}{2}\left(\beta_{1}+\beta_{2}+2 \beta_{3}\right)$ is a long root, and $\Phi \supset B_{3}$.

$\left(C_{3}\right) k=3$ and, up to reordering the roots $\beta_{i}$, we have that $\beta_{1}$ and $\beta_{2}$ are short and $\beta_{3}$ is long, $\frac{1}{2}\left(\beta_{1}+\beta_{2}+\beta_{3}\right)$ is a short root, and $\Phi \supset C_{3}$.

( $B_{2}$ long) $k=2, \beta_{1}$ and $\beta_{2}$ are short, $\beta_{1}+\beta_{2}$ is a long root, and $\Phi \supset B_{2}$.

$\left(B_{2}\right.$ short $) k=2, \beta_{1}$ and $\beta_{2}$ are long, $\frac{1}{2}\left(\beta_{1}+\beta_{2}\right)$ is a short root, and $\Phi \supset B_{2}$.

$\left(G_{2}\right.$ both $) k=2$, up to reordering the roots $\beta_{i}, \beta_{1}$ is long and $\beta_{2}$ is short, $\frac{1}{2}\left(\beta_{1}+\right.$ $\left.3 \beta_{2}\right)$ is a long root, $\frac{1}{2}\left(\beta_{1}+\beta_{2}\right)$ is a short root, and $\Phi \supset G_{2}$.

$\left(A_{1}\right) k=1$ and $n_{1}= \pm 1$.

Remark 3.6. The seven cases above can occur. In the list given below, we use the standard numbering $\left(\alpha_{1}, \ldots, \alpha_{k}\right)$ of the simple roots and we denote by $\theta$ the highest root. Moreover, in each case, if we observe that a linear combination $\sum q_{i} \beta_{i}$ is a root, then we let $e$ denote the nilpotent element $\sum e_{\beta_{i}}$. 
$\left(D_{4}\right)$ In type $D_{4}, \frac{1}{2}\left(\theta+\alpha_{1}+\alpha_{3}+\alpha_{4}\right) \in \Phi$. The height of $e$ is 4 .

$\left(B_{3}\right)$ In type $B_{3}, \frac{1}{2}\left(\theta+\alpha_{1}+2 \alpha_{3}\right) \in \Phi$. The height of $e$ is 4 .

$\left(C_{3}\right)$ In type $C_{3}, \frac{1}{2}\left(\alpha_{2}+\left(\alpha_{2}+\alpha_{3}\right)+\theta\right) \in \Phi$. The height of $e$ is 2 .

( $B_{2}$ long) In type $B_{2}, \alpha_{2}+\left(\alpha_{1}+\alpha_{2}\right) \in \Phi$. The height of $e$ is 2 .

( $B_{2}$ short) In type $B_{2}, \frac{1}{2}\left(\theta+\alpha_{1}\right)=\alpha_{1}+\alpha_{2} \in \Phi$. The height of $e$ is 2 .

$\left(G_{2}\right.$ both $)$ In type $G_{2}, \frac{1}{2}\left(\theta+3 \alpha_{1}\right)=3 \alpha_{1}+\alpha_{2} \in \Phi$ and $\frac{1}{2}\left(\theta+\alpha_{1}\right)=2 \alpha_{1}+\alpha_{2} \in \Phi$. The height of $e$ is 4 .

Note also that if $\beta_{1}, \beta_{2}, \beta_{3}$ is a set of three orthogonal roots which satisfy $\left(B_{3}\right)$, resp. $\left(C_{3}\right)$, then $\beta_{1}, \beta_{2}$ satisfy ( $B_{2}$ short), resp. ( $B_{2}$ long).

Proof of Remark 3.6. We justify the value of the height of $e$ indicated in each case.

In cases $\left(D_{4}\right),\left(B_{3}\right),\left(B_{2}\right.$ short $)$ and $\left(G_{2}\right.$ both $)$, no integral combination of the roots $\beta_{i}$ is a root. It follows that we can find an $\mathfrak{s l}_{2}$-triple $(e, h, f)$, whose element $h$ is the sum of the coroots. In case $\left(D_{4}\right)$, we have $h=\theta^{\vee}+\alpha_{1}^{\vee}+\alpha_{3}^{\vee}+\alpha_{4}^{\vee}$. This is equal to $2 \varpi_{1}^{\vee}-2 \varpi_{2}^{\vee}+2 \varpi_{3}^{\vee}+2 \varpi_{4}^{\vee}$, which is in the $W$-orbit of $2 \varpi_{2}^{\vee}$. The height of $e$ is then the value of this coweight on the highest root, namely $\left\langle\theta, 2 \varpi_{2}^{\vee}\right\rangle=4$.

The other cases are similar. In case $\left(B_{3}\right), h=2 \varpi_{1}^{\vee}-2 \varpi_{2}^{\vee}+2 \varpi_{3}^{\vee}$, which is equivalent to $2 \varpi_{2}^{\vee}$. In case $\left(B_{2}\right.$ short $), h=2 \varpi_{1}^{\vee}$. In case $\left(G_{2}\right.$ both), we have $\beta_{1}=\theta$ and $\beta_{2}=\alpha_{1}$. Then $h=\beta_{1}^{\vee}+\beta_{2}^{\vee}=2 \varpi_{1}^{\vee}-2 \varpi_{2}^{\vee}$, and $s_{2}(h)=2 \varpi_{2}^{\vee}$. Thus $e$ has height $\left\langle\theta, 2 \varpi_{2}^{\vee}\right\rangle=4$.

The cases $\left(C_{3}\right)$ and $\left(B_{2}\right.$ long) are addressed in Lemmas 3.11 and 3.12 .

Proof of Lemma 3.5. Let $\beta_{1}, \ldots, \beta_{k} \in \Phi$ and $n_{1}, \ldots, n_{k} \in \mathbb{Q} \backslash\{0\}$ be as in the statement of the lemma. Let $\beta=\sum n_{i} \beta_{i}$. If $i \in\{1, \ldots, k\}$, then $s_{\beta_{i}}(\beta)=-n_{i} \beta_{i}+$ $\sum_{j \neq i} n_{j} \beta_{j} \in \Phi$, so we may assume that $n_{i}>0$ for all $i$.

We consider the set $R=\left\{\beta_{1}, \ldots, \beta_{k},-\beta\right\}$ and the matrix $A=\left(\left\langle\alpha, \gamma^{\vee}\right\rangle\right)_{\alpha, \gamma \in R}$. By [GMP17, Lemma 5.2], this matrix is a generalized Cartan matrix of finite or affine type, and $-\beta$ is connected to all the other roots since $\left\langle\beta, \beta_{i}^{\vee}\right\rangle=2 n_{i}>0$ for all $i$. Moreover, we have $n_{i}=\frac{1}{2}\left\langle\beta, \beta_{i}^{\vee}\right\rangle \in \frac{1}{2} \mathbb{Z}$.

If $A$ has size 2 , then $\beta$ is a scalar multiple of $\beta_{1}$, and we are in case $\left(A_{1}\right)$ of the statement.

For $A$ being of size $k \geq 3$, we use the classification tables given at the end of chapter 4 in Kac83], which comprise twelve remaining cases that may fit the configuration of the root system.

(1) $A$ is of type $D_{4}^{(1)}$ and the Dynkin diagram of $A$ is

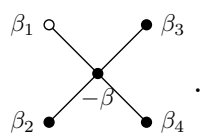
Then $\beta=\frac{1}{2}\left(\beta_{1}+\beta_{2}+\beta_{3}+\beta_{4}\right)$, and $\beta_{2},-\beta, \beta_{3}, \beta_{4}$ generate a subsystem of type $D_{4}$, so $\Phi \supset D_{4}$. We are in case $\left(D_{4}\right)$ of the statement.

(2) $A$ is of type $B_{3}^{(1)}$ and the Dynkin diagram of $A$ is

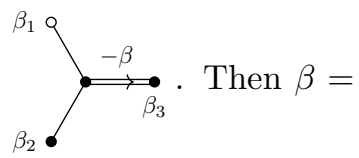
$\frac{1}{2}\left(\beta_{1}+\beta_{2}+2 \beta_{3}\right)$, and $\beta_{2},-\beta, \beta_{3}$ generate a subsystem of type $B_{3}$. Moreover, $\beta_{1}, \beta_{2}, \beta$ are long and $\beta_{3}$ is short. We are in case $\left(B_{3}\right)$. 
(3) $A$ is of type $A_{5}^{(2)}$ and the Dynkin diagram of $A$ is $\frac{1}{2}\left(\beta_{1}+\beta_{2}+\beta_{3}\right)$, and $\beta_{2},-\beta, \beta_{3}$ generate a subsystem of type $C_{3}$. Moreover, $\beta_{1}, \beta_{2}, \beta$ are short and $\beta_{3}$ is long. We are in case $\left(C_{3}\right)$.

(4) $A$ is of type $D_{3}^{(2)}$ and the Dynkin diagram of $A$ is $\beta=\beta_{1}+\beta_{2} ; \beta_{1}$ and $\beta_{2}$ have the same length and $\beta$ is longer. Moreover, $-\beta, \beta_{2}$ generate a root subsystem of type $B_{2}$. We are in case ( $B_{2}$ long).

(5) $A$ is of type $C_{2}^{(1)}$ and the Dynkin diagram of $A$ is $\underset{\beta_{1}}{\longrightarrow} \beta_{2}$. Then $\beta=\frac{1}{2}\left(\beta_{1}+\beta_{2}\right) ; \beta_{1}$ and $\beta_{2}$ have the same length and $\beta$ is shorter. Again, $\beta_{2},-\beta$ generate a subsystem of type $B_{2}$. We are in case ( $B_{2}$ short).

(6) $A$ is of type $G_{2}^{(1)}$ and the Dynkin diagram of $A$ is $\underset{\beta_{1}}{\longrightarrow} \underset{\beta_{2}}{\longrightarrow}$. Then $\beta=\frac{1}{2}\left(\beta_{1}+3 \beta_{2}\right) ; \beta_{1}, \beta$ are long roots, while $\beta_{2}$ is a short root as well as $\frac{1}{2}\left(\beta_{1}+\beta_{2}\right)$, since $s_{\beta}\left(\beta_{2}\right)=\beta_{2}-\beta=\frac{1}{2}\left(-\beta_{1}-\beta_{2}\right)$. Moreover, $-\beta, \beta_{2}$ generate a subsystem of type $G_{2}$. We are in case $\left(G_{2}\right.$ both).

(7) $A$ is of type $D_{4}^{(3)}$ and the Dynkin diagram of $A$ is $\beta=$ $\frac{1}{2}\left(\beta_{1}+\beta_{2}\right) ; \beta_{1}, \beta$ are short roots while $\beta_{2}$ is a long root as well as $\frac{1}{2}\left(3 \beta_{1}+\beta_{2}\right)$, since $s_{\beta}\left(\beta_{2}\right)=\frac{1}{2}\left(-3 \beta_{1}-\beta_{2}\right)$. Again, $\beta_{2},-\beta$ generate a subsystem of type $G_{2}$, and we are in case $\left(G_{2}\right.$ both).

(8) $A$ is of type $A_{4}^{(2)}$ and the Dynkin diagram of $A$ is three roots $\beta_{1}, \beta, \beta_{2}$ have pairwise distinct lengths. This is a contradiction. This case cannot occur.

We now consider the cases where $A$ is of finite type.

(9) $A$ is of type $D_{4}$ and the Dynkin diagram of $A$ is

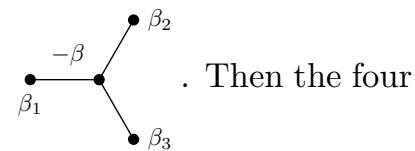
$\operatorname{roots} \beta_{1},-\beta, \beta_{2}, \beta_{3}$ have the same length, but $\beta=\frac{1}{2}\left(\beta_{1}+\beta_{2}+\beta_{3}\right)$. This is a contradiction. This case does not occur.

(10) $A$ is of type $B_{3}$ and the Dynkin diagram of $A$ is $\underset{\beta_{1}}{\longrightarrow} \underset{\beta_{2}}{\longrightarrow}$. Then $\beta=\frac{1}{2}\left(\beta_{1}+2 \beta_{2}\right)$ so $\|\beta\|^{2}=\frac{1}{4}\left\|\beta_{1}\right\|^{2}+\left\|\beta_{2}\right\|^{2}=\frac{3}{2}\left\|\beta_{2}\right\|^{2}$. This is again a contradiction, since we should have $\|\beta\|^{2}=2\left\|\beta_{2}\right\|^{2}$.

(11) $A$ is of type $C_{3}$ and the Dynkin diagram of $A$ is $\frac{1}{2}\left(\beta_{1}+\beta_{2}\right)$, so $\|\beta\|^{2}=\frac{3}{4}\left\|\beta_{2}\right\|^{2}$, and once again the lengths do not match.

(12) $A$ is of type $A_{3}$ and the Dynkin diagram of $A$ is $\underset{\beta_{1}}{\stackrel{-}{-\beta} \beta_{2}}$. Then $\beta=$ $\frac{1}{2}\left(\beta_{1}+\beta_{2}\right)$, which is again a contradiction since the three roots should have the same length.

It appears from Remark 3.6 that, if $\left(\theta_{k}\right)$ is a sequence of orthogonal roots which are not rationally orthogonal, the orbit of the nilpotent element $\sum e_{\theta_{k}}$ may nevertheless be spherical. We now give a full characterization of this property. 
Proposition 3.7. Assume that $G$ is simple. Let $\Theta=\left(\theta_{1}, \ldots, \theta_{r}\right)(r \geq 1)$ be a sequence of orthogonal roots and, for every $k \in\{1, \ldots, r\}$, let $e_{\theta_{k}} \in \mathfrak{g}_{\theta_{k}} \backslash\{0\}$. Then, the orbit $G \cdot e$ of the element $e=\sum_{k=1}^{r} e_{\theta_{k}}$ is spherical except exactly in the following cases (where we refer to the conditions listed in Lemma 3.5).

(1) $G$ has type $D$ or $E_{i}(i \in\{6,7,8\})$, and $\Theta$ contains four roots which satisfy condition $\left(D_{4}\right)$;

(2) $G$ has type $B$ or $F_{4}$, and $\Theta$ contains three roots which satisfy condition $\left(B_{3}\right)$ or two pairs of roots which satisfy condition ( $B_{2}$ short) with no common element;

(3) $G$ has type $G_{2}$, and $\Theta$ contains one pair of roots which satisfy condition $\left(G_{2}\right.$ both $)$.

Proof. If $G$ has type $A$, then $\theta_{1}, \ldots, \theta_{r}$ are always rationally orthogonal, and the result follows from Proposition 3.3 . In the other simply laced types $D, E_{6}, E_{7}, E_{8}$, the roots $\theta_{1}, \ldots, \theta_{r}$ are rationally orthogonal except if four of them satisfy condition $\left(D_{4}\right)$, in which case $e$ will have height $\geq 4$ in view of Remark 3.6. In type $G_{2}$, the roots are rationally orthogonal unless they satisfy $\left(G_{2}\right.$ both), in which case the claim that $G \cdot e$ is not spherical again follows from Remark 3.6. Finally, when $G$ has type $C, B$, or $F_{4}$, the result respectively follows from Lemmas 3.11, 3.12, or 3.13 .

In the following sequence of lemmas, we consider the notation of Proposition 3.7

Lemma 3.8. Let $i, j \in\{1, \ldots, r\}$ be such that $\theta_{i}-\theta_{j} \in \Phi$. Then, for every $k \neq j$, $\theta_{k}+\theta_{i}-\theta_{j} \notin \Phi$.

Proof. Let $\beta=\theta_{i}-\theta_{j} \in \Phi$ and let $k \neq i, j$. Since $\left\langle\theta_{k}, \theta_{i}^{\vee}\right\rangle=0$ and $\left\langle\theta_{k}, \theta_{j}^{\vee}\right\rangle=0$, we have $\left\langle\theta_{k}, \beta^{\vee}\right\rangle=0$. Since $\beta$ is a long root, it follows that $\theta_{k}+\beta \notin \Phi$. For length reasons, we must also have $2 \theta_{i}-\theta_{j} \notin \Phi$.

Lemma 3.9. Assume that there are $i, j \in\{1, \ldots, r\}$ such that $\theta_{i}-\theta_{j} \in \Phi$. Then

$$
\sum_{k=1}^{r} e_{\theta_{k}} \in G \cdot \sum_{k \neq i} e_{\theta_{k}} .
$$

Proof. Let $\beta=\theta_{i}-\theta_{j}$ and let $e_{\beta} \in \mathfrak{g}_{\beta} \backslash\{0\}$. Let $g \in G$ be the exponential of $e_{\beta}$. Then, by Lemma 3.8, $g \cdot e_{\theta_{k}}=e_{\theta_{k}}$ for $k \neq j$, and $g \cdot e_{\theta_{j}}=e_{\theta_{i}}+e_{\theta_{j}}$ (up to multiplying $e_{\beta}$ by some suitable scalar). The lemma follows.

Remark 3.10.

(a) The condition that $\theta_{i}-\theta_{j} \in \Phi$ of Lemma 3.9 can as well be replaced by $\theta_{i}+\theta_{j} \in \Phi$. Indeed, since $\theta_{i}$ and $\theta_{j}$ are orthogonal roots, then $\theta_{i}-\theta_{j}$ is a root if and only if $\theta_{i}+\theta_{j}=s_{\theta_{j}}\left(\theta_{i}-\theta_{j}\right)$ is a root.

(b) It follows from Lemma 3.9 that, for studying whether the $G$-orbit of $e=$ $\sum e_{\theta_{k}}$ is spherical or for computing the height of $e$, we may assume that no pair $\left(\theta_{i}, \theta_{j}\right)$ satisfies case $\left(B_{2}\right.$ long) of Lemma 3.5 Indeed, if case ( $B_{2}$ long) occurs within the sequence $\left(\theta_{k}\right)$, then this sequence may be replaced by a subsequence for which $\left(B_{2}\right.$ long) does not occur, and which yields a generator of the same nilpotent $G$-orbit.

Lemma 3.11. If $G$ has type $C$, then $e=\sum e_{\theta_{k}}$ is always spherical of height 2 . 
Proof. By Remark 3.10 we may assume that ( $B_{2}$ long) does not occur within the sequence $\left(\theta_{k}\right)$. Up to reordering the roots $\theta_{k}$, we may set an integer $l \in\{0, \ldots, r\}$ such that $\theta_{1}, \ldots, \theta_{l}$ are long and $\theta_{l+1}, \ldots, \theta_{r}$ are short. Up to the action of the Weyl group, we may assume that $\theta_{1}=2 \epsilon_{1}, \theta_{2}=2 \epsilon_{2}$, and so on up to $\theta_{l}=2 \epsilon_{l}$. If $l<r$, we may then assume that $\theta_{l+1}=\epsilon_{l+1}-\epsilon_{l+2}$. Since $\left(\theta_{l+1}, \theta_{l+2}\right)$ does not satisfy ( $B_{2}$ long), $\theta_{l+2}$ is of the form $\epsilon_{a} \pm \epsilon_{b}$ with $a, b>l+2$, and we may assume that $\theta_{l+2}=\epsilon_{l+3}-\epsilon_{l+4}$, and similarly for the next short roots. We can then check readily that $(\operatorname{ad} e)^{3}=0$ and invoke Proposition 3.1 .

Lemma 3.12. Assume that $G$ has type B. If one of the following conditions is satisfied:

(1) All the roots $\theta_{1}, \ldots, \theta_{r}$ are short;

(2) All the roots $\theta_{1}, \ldots, \theta_{r}$ are long and

- $r=2$, or

- no pair $\left(\theta_{i}, \theta_{j}\right)$ satisfies condition ( $B_{2}$ short) of Lemma 3.5 .

then e has height 2.

If one of the following conditions is satisfied:

(3) There are at least one short root and one long root among $\theta_{1}, \ldots, \theta_{r}$, and no pair $\left(\theta_{i}, \theta_{j}\right)$ satisfies ( $B_{2}$ short);

(4) $r \geq 3$, all the roots $\theta_{1}, \ldots, \theta_{r}$ are long, and there is exactly one pair $\left(\theta_{i}, \theta_{j}\right)$ which satisfies ( $B_{2}$ short),

then e has height 3.

In any other case, the height of $e$ is at least 4. In particular, $e$ is spherical if and only if one of the above conditions (1)-(4) is satisfied.

Proof. To prove the result, we use [Pa99, Theorem 2.3]. According to this result, a nonzero element $x \in \mathfrak{s o}(n) \subset \mathfrak{s l}(n)$ :

- has height 2 if $x^{2}=0$ or $\left(x^{3}=0\right.$ and $\left.\operatorname{rank} x=2\right)$;

- has height 3 if $x^{2} \neq 0, x^{3}=0$, rank $x \geq 3$, and rank $x^{2}=1$;

- has height at least 4 otherwise.

If all the roots $\theta_{1}, \ldots, \theta_{r}$ are short, then $e^{3}=0$ and rank $e=2$; so the height of $e$ is 2 in this case.

If all the roots $\theta_{k}$ are long but no pair $\left(\theta_{i}, \theta_{j}\right)$ satisfies ( $B_{2}$ short), then we have $e^{2}=0$; hence $e$ has height 2. If $r=2$ and the two roots are as in ( $B_{2}$ short), then $e^{3}=0$ and rank $e=2$, so the height of $e$ is again 2 .

If there are at least one short root and one long root, then $e^{2} \neq 0$ and rank $e \geq 4$. Moreover, if no pair $\left(\theta_{i}, \theta_{j}\right)$ satisfies ( $B_{2}$ short), then we have rank $e^{2}=1$, and so $e$ has height 3 in this case. If at least one pair $\left(\theta_{i}, \theta_{j}\right)$ satisfies $\left(B_{2}\right.$ short $)$, then rank $e^{2} \geq 2$, so that $e$ has height $\geq 4$.

Finally, assume that all the roots $\theta_{k}$ are long, $r \geq 3$, and exactly two of the roots are as in $\left(B_{2}\right.$ short). Then $e^{3}=0$, rank $e^{2}=1$, and rank $e \geq 4$; hence $e$ has height 3 . If there are at least two pairs of roots that satisfy $\left(B_{2}\right.$ short $)$, then we have rank $e^{2} \geq 2$; hence $e$ has height at least 4 . The last claim of the statement follows from Proposition 3.1

Lemma 3.13. If $G$ has type $F_{4}$, assume that ( $B_{2}$ long) does not occur within the sequence $\left(\theta_{k}\right)$. Then $e$ is spherical if and only if $r \leq 2$ or $(r=3$ and all the roots are long). 
Proof. One feature of type $F_{4}$ is that, whenever $\theta_{i}, \theta_{j}$ are orthogonal short roots, the sum $\theta_{i}+\theta_{j}$ is always a long root, that is, $\left(\theta_{i}, \theta_{j}\right)$ satisfies $\left(B_{2}\right.$ long). (Indeed, up to the action of the Weyl group, we may assume that $\theta_{i}$ is the highest short root, namely $\theta_{i}=\alpha_{1}+2 \alpha_{2}+3 \alpha_{3}+2 \alpha_{2}=\varpi_{4}$, and that $\theta_{j}=\alpha_{3}$.) Since we assume that the sequence $\left(\theta_{k}\right)$ contains no pair that satisfies $\left(B_{2}\right.$ long), it follows that $\left(\theta_{k}\right)$ contains at most one short root.

If $r=1$, then clearly $e$ is spherical. If $r=2$ and $\theta_{1}, \theta_{2}$ are both long, then up to the action of the Weyl group we may assume that $\theta_{1}=\theta=2 \alpha_{1}+3 \alpha_{2}+4 \alpha_{3}+2 \alpha_{4}$ is the highest root and $\theta_{2}=\alpha_{2}+2 \alpha_{3}+2 \alpha_{4}$. Then, since no integral combination of $\theta_{1}$ and $\theta_{2}$ is a root, an $\mathfrak{s l}_{2}$-triple for $e$ is given by $h=\theta_{1}^{\vee}+\theta_{2}^{\vee}$ and $f=f_{\theta_{1}}+f_{\theta_{2}}$ for some $f_{\theta_{1}} \in \mathfrak{g}_{-\theta_{1}}, f_{\theta_{2}} \in \mathfrak{g}_{-\theta_{2}}$. One computes that $\theta_{1}^{\vee}+\theta_{2}^{\vee}=\varpi_{4}^{\vee}$ is dominant, so the height of $e$ is $\theta\left(\varpi_{4}^{\vee}\right)=2$.

If $r=2$ and $\theta_{1}$ is long and $\theta_{2}$ is short, once again, the action of $W$ allows us to assume that $\theta_{1}=2 \alpha_{1}+3 \alpha_{2}+4 \alpha_{3}+2 \alpha_{4}$ and $\theta_{2}=\alpha_{2}+2 \alpha_{3}+\alpha_{4}$. The same argument holds, and $e$ belongs to an $\mathfrak{s l}_{2}$-triple $(e, h, f)$ such that $h=\theta_{1}^{\vee}+\theta_{2}^{\vee}$. Then, we can see that $h=-\varpi_{1}^{\vee}+\varpi_{3}^{\vee}=s_{1} s_{2}\left(\varpi_{2}^{\vee}\right)$. Hence, the height of $e$ is $\theta\left(\varpi_{2}^{\vee}\right)=3$.

If $r=3$, since there is at most one short root, we may assume that $\theta_{1}$ and $\theta_{2}$ are long, and that we have again $\theta_{1}=2 \alpha_{1}+3 \alpha_{2}+4 \alpha_{3}+2 \alpha_{4}$ and $\theta_{2}=\alpha_{2}+2 \alpha_{3}+2 \alpha_{4}$. Then $\theta_{1}^{\vee}=\varpi_{1}^{\vee}$ and $\theta_{2}^{\vee}=-\varpi_{1}^{\vee}+\varpi_{4}^{\vee}$, so $\theta_{3}$ must be a linear combination of $\alpha_{2}$ and $\alpha_{3}$. Up to the action of $W$, there are two cases: if $\theta_{3}$ is short, then we may assume that $\theta_{3}=\alpha_{2}+\alpha_{3}$, and then $\theta_{1}^{\vee}+\theta_{2}^{\vee}+\theta_{3}^{\vee}=-2 \varpi_{1}^{\vee}+2 \varpi_{2}^{\vee}=s_{1}\left(2 \varpi_{1}^{\vee}\right)$. Thus $e$ has height $\theta\left(2 \varpi_{1}^{\vee}\right)=4$, and it is not spherical. If $\theta_{3}$ is long, we may assume that $\theta_{3}=\alpha_{2}+2 \alpha_{3}$. Then $\theta_{1}^{\vee}+\theta_{2}^{\vee}+\theta_{3}^{\vee}=-\varpi_{1}^{\vee}+\varpi_{3}^{\vee}=s_{1} s_{2}\left(\varpi_{2}^{\vee}\right)$, so $e$ has height 3 and it is spherical in this case.

If $r=4$, we know that there are at least three long roots. So, as in the previous paragraph, we may assume that $\theta_{1}=2 \alpha_{1}+3 \alpha_{2}+4 \alpha_{3}+2 \alpha_{4}, \theta_{2}=\alpha_{2}+2 \alpha_{3}+2 \alpha_{4}$ and $\theta_{3}=\alpha_{2}+2 \alpha_{3}$. Then $\theta_{4}$ must be equal to $\pm \alpha_{2}$, say $\alpha_{2}$. We recover the coweight $\theta_{1}^{\vee}+\theta_{2}^{\vee}+\theta_{3}^{\vee}+\theta_{4}^{\vee}=-2 \varpi_{1}^{\vee}+2 \varpi_{2}^{\vee}=s_{1}\left(2 \varpi_{1}^{\vee}\right)$. Hence $e$ has height 4 , so it is not spherical.

A convenient way to produce orthogonal roots is to use Harish-Chandra chain cascade of roots in the version defined by Kostant Ko12, §1]. However, in this way we get a sequence of strongly orthogonal roots [Ko12, Lemma 1.6] that may not be a sequence of rationally orthogonal roots. For example, in type $E_{6},\left(\theta, \alpha_{1}+\alpha_{3}+\right.$ $\left.\alpha_{4}+\alpha_{5}+\alpha_{6}, \alpha_{3}+\alpha_{4}+\alpha_{5}, \alpha_{4}\right)$ is such an example, corresponding to case $\left(D_{4}\right)$ in Lemma 3.5. The examples for cases ( $B_{2}$ short) and $\left(G_{2}\right.$ both) given in Remark 3.6 are also chain cascades, and one can check that these three cases are the only cases of Lemma 3.5 that can occur in a chain cascade. Our final remark in this line of ideas is the following:

Remark 3.14. Let $\left(\theta_{k}\right)$ be a sequence obtained by chain cascade. If the nilpotent element $\sum e_{\theta_{k}}$ has height 2 , then the element $\sum \theta_{k}^{\vee}$ is dominant, by GMP20, Proposition 3.10].

\section{Example 3.15.}

(a) Assume that $G=\mathrm{SL}_{n}(\mathbb{K})$. A nilpotent matrix $e \in \mathfrak{s l}_{n}(\mathbb{K})$ belongs to a spherical nilpotent orbit if and only if $e^{2}=0$ (see Pa94]). For every $r \in\left\{0, \ldots,\left\lfloor\frac{n}{2}\right\rfloor\right\}$, the set $\mathcal{O}^{(r)}:=\left\{e \in \mathfrak{s l}_{n}(\mathbb{K}): e^{2}=0\right.$, rank $\left.e=r\right\}$ consists of a single $\mathrm{SL}_{n}(\mathbb{K})$-orbit. Let $\Phi=\left\{\epsilon_{i}-\epsilon_{j}: 1 \leq i \neq j \leq n\right\}$ be the usual root system. Then for every 
permutation $\sigma$ of $\{1, \ldots, r\}$, the roots

$$
\theta_{i}^{\sigma}:=\epsilon_{i}-\epsilon_{n+1-\sigma(i)} \quad(\text { for } i=1, \ldots, r)
$$

form a sequence of rationally orthogonal roots, and for every choice of root vectors $e_{\theta_{i}^{\sigma}} \in \mathfrak{g}_{\theta_{i}^{\sigma}} \backslash\{0\}$, the element

$$
e_{\sigma}:=\sum_{i=1}^{r} e_{\theta_{i}^{\sigma}}
$$

is a representative of $\mathcal{O}^{(r)}$ which is of the form described in Proposition 3.3(b), i.e., the corresponding coweight $\sum_{i=1}^{r}\left(\theta_{i}^{\sigma}\right)^{\vee}$ is dominant. Thus the sequence of rationally orthogonal roots described in Proposition 3.3(b) is not unique for each orbit. Note however that the coweight $\sum_{i=1}^{r}\left(\theta_{i}^{\sigma}\right)^{\vee}$ is independent of $\sigma$, which agrees with Remark 3.4. For $\sigma=\mathrm{id}$, the sequence $\left(\theta_{i}^{\text {id }}\right)$ is a chain cascade.

(b) As another example, we give the sequences of roots $\left(\theta_{i}\right)$ given by Proposition 3.3 in the case of the exceptional group of type $E_{7}$. In view of CMcG93, table p. 130], $\mathfrak{g}$ has five nontrivial spherical nilpotent orbits in this case, whose types are respectively $A_{1}, 2 A_{1}, 3 A_{1}, 3 A_{1}, 4 A_{1}$. We use the following numbering of the simple roots/vertices of the Dynkin diagram:

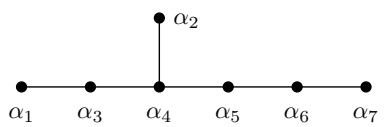

For $i \in\{1, \ldots, 7\}$, we set $s_{i}=s_{\alpha_{i}}$. Note that in a simply laced case (as $E_{7}$ ), two roots are orthogonal if and only if they are strongly orthogonal.

Let $\theta_{1}$ be the highest root, which is equal to the fundamental weight $\varpi_{1}$. Thus, we have $\theta_{1}^{\vee}=\varpi_{1}^{\vee}$. The root vector $e_{\theta_{1}}$ belongs to the orbit of type $A_{1}$ (which is the minimal nilpotent orbit of $\mathfrak{g}$ ).

Let $\theta_{2}$ be the highest root which is orthogonal to $\theta_{1}$, namely $\alpha_{2}+\alpha_{3}+2 \alpha_{4}+$ $2 \alpha_{5}+2 \alpha_{6}+\alpha_{7}$. It is also equal to $-\varpi_{1}+\varpi_{6}$, so that $\theta_{1}^{\vee}+\theta_{2}^{\vee}=\varpi_{6}^{\vee}$. The vector $e_{\theta_{1}}+e_{\theta_{2}}$ belongs to the orbit of type $2 A_{1}$.

The orthogonal of $\theta_{1}$ and $\theta_{2}$ consists of roots with no term in $\alpha_{1}$ nor $\alpha_{6}$. Since this orthogonal is disconnected, we have three choices for the third root in the chain cascade according to Kostant's algorithm Ko12. One is $\theta_{3}^{\prime \prime}=\alpha_{7}=2 \varpi_{7}-\varpi_{6}$. By Lemma 3.5. $\left(\theta_{1}, \theta_{2}, \theta_{3}^{\prime \prime}\right)$ is a set of rationally orthogonal roots, and $\theta_{1}^{\vee}+\theta_{2}^{\vee}+\left(\theta_{3}^{\prime \prime}\right)^{\vee}=$ $2 \varpi_{7}^{\vee}$. The vector $e_{\theta_{1}}+e_{\theta_{2}}+e_{\theta_{3}^{\prime \prime}}$ belongs to the nilpotent orbit labeled $\left(3 A_{1}\right)^{\prime \prime}$ in CMcG93, table p.130].

Note that up to now, the heights of the nilpotent elements were 2 and, as predicted by Remark 3.14, the coweights $\sum_{i} \theta_{i}^{\vee}$ were dominant. We now consider some nilpotent elements of height 3 and $\sum_{i} \theta_{i}^{\vee}$ will not be dominant.

The second choice for the third root in the chain cascade is $\theta_{3}^{\prime}=\alpha_{2}+\alpha_{3}+2 \alpha_{4}+\alpha_{5}$. Then, we have $\theta_{1}^{\vee}+\theta_{2}^{\vee}+\left(\theta_{3}^{\prime}\right)^{\vee}=-\varpi_{1}^{\vee}+\varpi_{4}^{\vee}$, which is not a dominant coweight. In this case, we consider the sequence $s_{3} s_{1}\left(\theta_{1}\right), s_{3} s_{1}\left(\theta_{2}\right), s_{3} s_{1}\left(\theta_{3}^{\prime}\right)$, which is also a set of rationally orthogonal roots, and which satisfies $s_{3} s_{1}\left(\theta_{1}\right)^{\vee}+s_{3} s_{1}\left(\theta_{2}\right)^{\vee}+s_{3} s_{1}\left(\theta_{3}^{\prime}\right)^{\vee}=$ $\varpi_{3}^{\vee}$. The vector $e_{s_{3} s_{1}\left(\theta_{1}\right)}+e_{s_{3} s_{1}\left(\theta_{2}\right)}+e_{s_{3} s_{1}\left(\theta_{3}^{\prime}\right)}$ belongs to the orbit labeled $\left(3 A_{1}\right)^{\prime}$ in CMcG93, table p. 130].

Finally, the three possible chain cascades of length 4 are $\left(\theta_{1}, \theta_{2}, \theta_{3}^{\prime}, \alpha_{i}\right)$ with $i \in\{2,3,5\}$. We have $\theta_{1}^{\vee}+\theta_{2}^{\vee}+\left(\theta_{3}^{\prime}\right)^{\vee}+\alpha_{2}^{\vee}=-\varpi_{1}^{\vee}+2 \varpi_{2}^{\vee}$ (which is not a dominant coweight). Letting $s=s_{7} s_{6} s_{5} s_{4} s_{3} s_{1}$, we get $s\left(\theta_{1}^{\vee}\right)+s\left(\theta_{2}^{\vee}\right)+s\left(\left(\theta_{3}^{\prime}\right)^{\vee}\right)+s\left(\alpha_{2}^{\vee}\right)=$ 
$\varpi_{2}^{\vee}+\varpi_{7}^{\vee}$ (which is a dominant coweight). The element $e_{s\left(\theta_{1}\right)}+e_{s\left(\theta_{2}\right)}+e_{s\left(\theta_{3}^{\prime}\right)}+e_{s\left(\alpha_{2}\right)}$ belongs to the orbit labeled $4 A_{1}$.

In the same way, we have $\theta_{1}^{\vee}+\theta_{2}^{\vee}+\left(\theta_{3}^{\prime}\right)^{\vee}+\alpha_{5}^{\vee}=-\varpi_{1}^{\vee}+2 \varpi_{5}^{\vee}-\varpi_{6}^{\vee}=$ $s_{1} s_{3} s_{4} s_{2} s_{6} s_{7}\left(\varpi_{2}^{\vee}+\varpi_{7}^{\vee}\right)$; hence we recover the same orbit labeled $4 A_{1}$.

The situation is different for the last possible chain cascade $\left(\theta_{1}, \theta_{2}, \theta_{3}^{\prime}, \alpha_{3}\right)$. These roots are not rationally orthogonal as they satisfy condition $\left(D_{4}\right)$ of Lemma 3.5 . In fact, in this case, we have $\theta_{1}^{\vee}+\theta_{2}^{\vee}+\left(\theta_{3}^{\prime}\right)^{\vee}+\alpha_{3}^{\vee}=-2 \varpi_{1}^{\vee}+2 \varpi_{3}^{\vee}=s_{1}\left(2 \varpi_{1}^{\vee}\right)$; hence the nilpotent element $e_{\theta_{1}}+e_{\theta_{2}}+e_{\theta_{3}^{\prime}}+e_{\alpha_{3}}$ belongs to the orbit labeled $A_{2}$ in [CMcG93, table p. 130] (which is of height 4).

\section{Symmetric Subgroup Associated to A SPhericAl nilpotent orbit}

In this section, we assume that the orbit $G \cdot e$ is spherical and relate the subgroups $L_{Z}^{0}$ and $L_{Z}$ of $Z$ to a symmetric subgroup of $L$. We use the notation introduced in Section 1

Definition 4.1. Let $e \in \mathfrak{g}$ be a nilpotent element and let $\mathfrak{s}=(e, h, f)$ be a standard triple. As in Section 1, we consider a cocharacter $\tau: \mathbb{K}^{*} \rightarrow G$ such that $\tau^{\prime}(1)=h$. We assume that $h$ belongs to the Lie algebra $\mathfrak{t}=\operatorname{Lie}(T)$ of the standard torus $T \subset G$, so that $\tau\left(\mathbb{K}^{*}\right)$ is contained in $T$.

We consider the Lie algebra $\mathfrak{s l}_{2}(\mathfrak{s})$ linearly generated by $e, h$, and $f$, and we consider the subgroup $\mathrm{SL}_{2}(\mathfrak{s}) \subset G$ with Lie algebra $\mathfrak{s l}_{2}(\mathfrak{s})$. The torus $\tau\left(\mathbb{K}^{*}\right)$ is a maximal torus of $\mathrm{SL}_{2}(\mathfrak{s})$.

We denote by $n_{\mathfrak{s}} \in \mathrm{SL}_{2}(\mathfrak{s})$ an element in the normalizer of $\tau\left(\mathbb{K}^{*}\right)$, and not in $\tau\left(\mathbb{K}^{*}\right)$ (thus, a representative of the nontrivial element of the Weyl group of $\mathrm{SL}_{2}(\mathfrak{s})$ ). By a standard calculation in $\mathrm{SL}_{2}(\mathbb{K})$, we get

$$
n_{\mathfrak{s}} \tau(t) n_{\mathfrak{s}}^{-1}=\tau\left(t^{-1}\right) \text { for all } t \in \mathbb{K}^{*} .
$$

We denote by $\sigma: G \rightarrow G$ the conjugation by $n_{\mathfrak{s}}$ and we use the same notation for the adjoint action of $n_{\mathfrak{s}}$ on $\mathfrak{g}$.

We make a first observation:

\section{Lemma 4.2.}

(a) The map $\sigma$ preserves the subgroup $L$; in fact, $\sigma: L \rightarrow L$ is an involution.

(b) We have $Z \cap L \subset L^{\sigma}$ and $\mathfrak{z}_{\mathfrak{g}}(e) \cap \mathfrak{l} \subset \mathfrak{l}^{\sigma}$.

Proof. (a) In view of (1), $\sigma$ preserves the torus $\tau\left(\mathbb{K}^{*}\right)$; hence $\sigma$ also preserves the centralizer of $\tau\left(\mathbb{K}^{*}\right)$, which is precisely $L$. Since the Weyl group of $\mathrm{SL}_{2}(\mathfrak{s})$ has only two elements, we must have $n_{\mathfrak{s}}^{2} \in \tau\left(\mathbb{K}^{*}\right)$; hence $n_{\mathfrak{s}}^{2}$ is contained in the center of $L$. This implies that $\sigma^{2}(g)=g$ for all $g \in L$.

(b) We have $Z \cap L=Z_{G}(e) \cap Z_{G}(h)=Z_{G}\left(\mathfrak{s l}_{2}(\mathfrak{s})\right)$ (see [CMcG93, Lemma 3.4.4]). Moreover $Z_{G}\left(\mathfrak{s l}_{2}(\mathfrak{s})\right)=Z_{G}\left(\mathrm{SL}_{2}(\mathfrak{s})\right)$, hence $Z \cap L=Z_{G}\left(\mathrm{SL}_{2}(\mathfrak{s})\right)$. Since $n_{\mathfrak{s}} \in \mathrm{SL}_{2}(\mathfrak{s})$, this implies that $Z \cap L \subset L^{\sigma}$. This inclusion yields the inclusion of Lie subalgebras $\mathfrak{z} \mathfrak{g}(e) \cap \mathfrak{l} \subset \mathfrak{l}^{\sigma}$.

Remark 4.3. Whereas the map $\sigma: G \rightarrow G$ a priori depends on the choice of $n_{\mathfrak{s}}$ within a $\tau\left(\mathbb{K}^{*}\right)$-coset, its restriction $\sigma: L \rightarrow L$ is independent of this choice, because $L=Z_{G}(\tau)$.

Lemma 4.4 will be used to exclude particular configurations of roots in our root system $\Phi$. Recall that in a Kac-Moody root system, a real root is by definition a root which is in the Weyl group orbit of a simple root. 


\section{Lemma 4.4.}

(a) Let $\phi$ be the set of roots of a root system. Let $\beta_{1}, \ldots, \beta_{l} \in \phi$ be linearly independent. Assume that the matrix $\left(\left\langle\beta_{i}, \beta_{j}^{\vee}\right\rangle\right)_{1 \leq i, j \leq l}$ is the (generalized) Cartan matrix of a given type $X_{l}$. Then the intersection of $\phi$ with the linear space generated by $\beta_{1}, \ldots, \beta_{l}$ contains at least as many roots as the number of real roots in the root system of type $X_{l}$.

(b) In particular, if $\phi$ is of finite type, $\theta_{1}, \ldots, \theta_{k} \in \phi$ are rationally orthogonal, and $\alpha \in \phi \backslash\left\{ \pm \theta_{t}\right\}_{t=1}^{k}$, then the matrix $\left(\left\langle\beta_{i}, \beta_{j}^{\vee}\right\rangle\right)_{1 \leq i, j \leq k+1}$ with $\beta_{i} \in\left\{ \pm \theta_{i}\right\}$ for $i \leq k$ and $\beta_{k+1}=\alpha$ cannot be a Cartan matrix of non-finite type.

Proof. Let $\alpha_{1}, \ldots, \alpha_{l}$ form a basis of the root system of type $X_{l}$. Let $\alpha=\sum_{i=1}^{l} n_{i} \alpha_{i}$ be a real root in the root system of type $X_{l}$, and let $i_{1}, \ldots, i_{\ell}, j$ be such that $\alpha=s_{\alpha_{i_{1}}} \cdots s_{\alpha_{i_{\ell}}}\left(\alpha_{j}\right)$. Then $s_{\beta_{i_{1}}} \cdots s_{\beta_{i_{\ell}}}\left(\beta_{j}\right)$ is equal to $\sum_{i=1}^{l} n_{i} \beta_{i}$, because the computation only depends on the Cartan matrix. Therefore, every real root $\alpha$ has a corresponding element $\sum_{i=1}^{l} n_{i} \beta_{i}$ in $\phi \cap \operatorname{Span}\left\{\beta_{1}, \ldots, \beta_{l}\right\}$. This shows (a).

Part (b) of the lemma follows because, due to the assumption of rational orthogonality, the roots $\theta_{1}, \ldots, \theta_{k}, \alpha$ are linearly independent.

Proposition 4.5. Let $e=\sum_{i=1}^{r} e_{\theta_{i}}$ be as in Proposition $3.3(\mathrm{a})$; in particular its orbit $G \cdot e$ is spherical. Then, we have $\mathfrak{z}_{\mathfrak{g}}(e) \cap \mathfrak{l}=\mathfrak{l}^{\sigma}$ and $L_{Z}^{0}=\left(L^{\sigma}\right)^{0} \subset L_{Z} \subset L^{\sigma}$.

Proof. Recall that $L$ is the centralizer of $\tau\left(\mathbb{K}^{*}\right)$. Thus,

$$
\mathfrak{l}=\mathfrak{t} \oplus \bigoplus_{\substack{\alpha \in \Phi \\ \text { s.t. }\langle\alpha, h\rangle=0}} \mathfrak{g}_{\alpha} .
$$

We claim that

$$
\sigma(T)=T, \quad \text { i.e., } n_{\mathfrak{s}} \text { belongs to } N_{G}(T) .
$$

Indeed, we have already noted that $\sigma$ preserves the torus $\tau\left(\mathbb{K}^{*}\right)$. Let $S=\bigcap_{i} \operatorname{ker} \theta_{i}$. Then $S \subset Z \cap T \subset Z \cap L$, which implies that $\sigma$ fixes every element of $S$ (by Lemma 4.2). Thereby $\sigma$ preserves the torus $S \tau\left(\mathbb{K}^{*}\right)$ and so it also preserves its centralizer. As noted in the proof of Proposition 3.3, we have $Z_{G}\left(S \tau\left(\mathbb{K}^{*}\right)\right)=T$. Whence (2).

Relation (2) implies that $\sigma$ induces an involution on the root system $\Phi$ (that we denote by the same letter). In fact this action coincides with the action of the Weyl group element

$$
s_{\theta_{1}} \cdots s_{\theta_{r}}: \alpha \mapsto \alpha-\sum_{i}\left\langle\alpha, \theta_{i}^{\vee}\right\rangle \theta_{i}
$$

We have the following equality:

$$
\mathfrak{l}^{\sigma}=\mathfrak{t}^{\sigma} \oplus \bigoplus_{\alpha} \mathfrak{g}_{\alpha}^{\sigma} \oplus \bigoplus_{\{\alpha, \beta\}}\left(\mathfrak{g}_{\alpha} \oplus \mathfrak{g}_{\beta}\right)^{\sigma},
$$

where the former sum is over the roots $\alpha$ of $L$ such that $\sigma(\alpha)=\alpha$, and the latter sum is over the pairs $\{\alpha, \beta\}$ of roots of $L$ with $\beta=\sigma(\alpha) \neq \alpha$. Let $\alpha$ be a root of $L$. We consider two cases.

First, if $\sigma(\alpha)=\alpha$, then for all $i$ we have $\left\langle\alpha, \theta_{i}^{\vee}\right\rangle=0$. Assume first that there exists $i$ such that $\alpha+\theta_{i}$ is a root. Then $\alpha-\theta_{i}=s_{\theta_{i}}\left(\alpha+\theta_{i}\right)$ is also a root. We claim that there can be only one such integer $i$. In fact, let us assume to the contrary that there are two integers $i, j$ such that $\alpha+\theta_{i}$ and $\alpha+\theta_{j}$ are roots. Then, considering the $\left(\mathfrak{s l}_{2}\left(\theta_{i}\right) \times \mathfrak{s l}_{2}\left(\theta_{j}\right)\right)$-module generated by $\mathfrak{g}_{\alpha}$, we deduce that $\alpha+\theta_{i}+\theta_{j}$ is a root. 
But then, the square lengths of $\alpha, \alpha+\theta_{i}$ and $\alpha+\theta_{i}+\theta_{j}$ are three different numbers, a contradiction.

Let $i$ be the unique integer such that $\alpha+\theta_{i}$ is a root. It follows that the $\mathfrak{s l}_{2}\left(\theta_{i}\right)$ module generated by $\mathfrak{g}_{\alpha}$ is isomorphic to the adjoint module $\mathfrak{s l}_{2}\left(\theta_{i}\right)$. Thus the same holds for the corresponding $\mathrm{SL}_{2}\left(\theta_{i}\right)$-module. By a direct computation in $\mathrm{SL}_{2}(\mathbb{K})$, we deduce that the restriction of the nontrivial element of the Weyl group of $\mathrm{SL}_{2}\left(\theta_{i}\right)$ to $\mathfrak{g}_{\alpha}$ is $-\mathrm{id}_{\mathfrak{g}_{\alpha}}$. Since the elements of the Weyl group of $\mathrm{SL}_{2}\left(\theta_{j}\right)$ act trivially on $\mathfrak{g}_{\alpha}$ for $j \neq i$, we deduce that $\sigma$ acts as $-\operatorname{id}_{\mathfrak{g}_{\alpha}}$ on $\mathfrak{g}_{\alpha}$. Therefore, $\mathfrak{g}_{\alpha}^{\sigma}=\{0\}$ in this case.

If for all integers $i, \alpha+\theta_{i}$ is not a root, we get $\mathfrak{g}_{\alpha} \subset \mathfrak{z}_{\mathfrak{g}}(e)$. In both cases, we deduce from Lemma 4.2 that we have $\mathfrak{z}_{\mathfrak{g}}(e) \cap \mathfrak{g}_{\alpha}=\mathfrak{g}_{\alpha}^{\sigma}$.

Second, if $\sigma(\alpha) \neq \alpha$, let us set $\beta=\sigma(\alpha)$. First, assume that for all integers $i$, we have $\left|\left\langle\alpha, \theta_{i}^{\vee}\right\rangle\right| \leq 1$. Then, the number of integers $i$ such that $\left\langle\alpha, \theta_{i}^{\vee}\right\rangle \neq 0$ is 2. Indeed, it must be even since $\left\langle\alpha, \theta_{i}^{\vee}\right\rangle$ belongs to $\{-1,0,1\}$ for all $i$, and $\sum_{i}\left\langle\alpha, \theta_{i}^{\vee}\right\rangle=0$. Moreover, it must be less than 4 because, otherwise, $\alpha$ and four such roots would yield a Cartan matrix $\left(\left\langle\beta_{i}, \beta_{j}^{\vee}\right\rangle\right)_{1 \leq i, j \leq 5}$ (as in Lemma 4.4(b)) of non-finite type, contradicting Lemma 4.4. Assume now that there exists an integer $i$ such that $\left|\left\langle\alpha, \theta_{i}^{\vee}\right\rangle\right| \geq 2$. Then, since $\sum_{j}\left\langle\alpha, \theta_{j}^{\vee}\right\rangle=0$, there are two cases. Either there is another integer $j \neq i$ such that $\left|\left\langle\alpha, \theta_{j}^{\vee}\right\rangle\right| \geq 2$, in which case $\theta_{i}, \theta_{j}, \alpha$ yield (as before) a Cartan matrix of non-finite type, which is absurd in view of Lemma 4.4(b). Or there are at least two integers $j$ such that $\left|\left\langle\alpha, \theta_{j}^{\vee}\right\rangle\right|=1$ and, once again, this is absurd by virtue of Lemma 4.4(b).

We have therefore shown that there are $i, j \in\{1, \ldots, r\}$ such that $\left\langle\alpha, \theta_{i}^{\vee}\right\rangle=1$, $\left\langle\alpha, \theta_{j}^{\vee}\right\rangle=-1$, and $\left\langle\alpha, \theta_{k}^{\vee}\right\rangle=0$ for all $k \neq i, j$. We have $\beta=\sigma(\alpha)=\alpha-\theta_{i}+\theta_{j}$. Moreover, $s_{\theta_{i}}(\alpha)=\alpha-\theta_{i}$ is a root, so $\alpha+\theta_{i}$ is not a root. Note also that if $k \neq i, j$, then $\alpha+\theta_{k}$ cannot be a root; otherwise $\alpha+\theta_{k},-\theta_{k},-\theta_{i}, \theta_{j}$ would define a Cartan matrix of type $B_{3}^{(1)}$, a contradiction with Lemma 4.4(b). Similarly, $\beta+\theta_{k}$ is not a root unless $k=i$. It follows that

$$
\left[e,\left(\mathfrak{g}_{\alpha} \oplus \mathfrak{g}_{\beta}\right)\right]=\mathfrak{g}_{\alpha+\theta_{j}}=\mathfrak{g}_{\beta+\theta_{i}} .
$$

Thus, $\operatorname{dim} \mathfrak{z}_{\mathfrak{g}}(e) \cap\left(\mathfrak{g}_{\alpha} \oplus \mathfrak{g}_{\beta}\right)=1$. On the other hand, $\left(\mathfrak{g}_{\alpha} \oplus \mathfrak{g}_{\beta}\right)^{\sigma}$ is also 1-dimensional. By Lemma 4.2, we get $\left(\mathfrak{g}_{\alpha} \oplus \mathfrak{g}_{\beta}\right)^{\sigma}=\mathfrak{z}_{\mathfrak{g}}(e) \cap\left(\mathfrak{g}_{\alpha} \oplus \mathfrak{g}_{\beta}\right)$.

Finally, we claim that $\mathfrak{t}^{\sigma}=\mathfrak{z}_{\mathfrak{g}}(e) \cap \mathfrak{t}$. In fact, $\mathfrak{t}^{\sigma}$ is the orthogonal of all the roots $\theta_{i}$, because $\sigma$ is the product of the reflections defined by $\theta_{1}, \ldots, \theta_{r}$. Since $e_{\theta_{i}}$ has weight $\theta_{i}, \mathfrak{z}_{\mathfrak{g}}(e) \cap \mathfrak{t}$ is also the orthogonal of the roots $\theta_{i}$. Whence the claimed equality.

Altogether we have shown the desired equality $\mathfrak{z}_{\mathfrak{g}}(e) \cap \mathfrak{l}=\mathfrak{l}^{\sigma}$. This equality implies $L_{Z}^{0}=\left(L^{\sigma}\right)^{0}$. The inclusion $L_{Z} \subset L^{\sigma}$ is already noted in Lemma 4.2.

Remark 4.6. The fact that $\mathfrak{z}_{\mathfrak{g}}(e) \cap \mathfrak{l}$ is a symmetric subalgebra of $\mathfrak{l}$ is already shown in [Pa94, §3.3] (with a different proof). We also refer to [BC10, Appendix B] where the symmetric pairs $\left(L, L^{\sigma}\right)$ corresponding to the nilpotent elements $e$ of height 3 are explicitly described.

\section{Example 4.7.}

(a) We first consider the case of nilpotent orbits in type $A_{l}$. Let $e$ be a nilpotent element of height 2 and rank $r$ in the Lie algebra $\mathfrak{s l}_{l+1}$ : this means that the endomorphism $e$ satisfies $e^{2}=0$ and that the partition giving the size of the Jordan blocks is $\left(2^{r}, 1^{l+1-2 r}\right)$. The coweight $h$ in an $\mathfrak{s l}_{2}$-triple $(e, h, f)$ is $\varpi_{r}^{\vee}+\varpi_{l+1-r}^{\vee}$ (see also Example 3.15(a)). Thus $L$ is the standard Levi subgroup corresponding 
to the root subsystem of type $A_{r-1} \times A_{r-1} \times A_{l-2 r}$ generated by the simple roots $\alpha_{1}, \ldots, \alpha_{r-1}, \alpha_{r+1}, \ldots, \alpha_{l-r}, \alpha_{l+2-r}, \ldots, \alpha_{l}$.

The element $e$ can be described as a sum of root vectors using the procedure given in Proposition 3.3, it is in the orbit of the sum $e_{\theta_{1}}+\cdots+e_{\theta_{r}}$ where $\theta_{i}=$ $\alpha_{i}+\cdots+\alpha_{l+1-i}$. Moreover, the involution is given by the nontrivial Weyl group element of the subgroup with Lie algebra linearly generated by $(e, h, f)$. This element acts on the roots as the element $w=s_{\theta_{1}} \cdots s_{\theta_{r}}$ of the Weyl group. Thus, we have $w\left(\alpha_{i}\right)=\alpha_{i}-\theta_{i}+\theta_{i+1}=-\alpha_{l+1-i}$ if $1 \leq i \leq r-1$, and $w\left(\alpha_{i}\right)=\alpha_{i}$ if $r+1 \leq i \leq l-r$. In this way, we recover the known fact that $L$ is of type $A_{r-1} \times A_{r-1} \times A_{l-2 r}$ while $L^{\sigma}$ is of type $A_{r-1} \times A_{l-2 r}$ (with a diagonal embedding of $A_{r-1}$ in the factor $\left.A_{r-1} \times A_{r-1}\right)$.

(b) We now consider the case of the nilpotent orbit labeled $3 A_{1}$ in type $E_{6}$, which corresponds to the dominant coweight $\varpi_{4}^{\vee}$ (see [CMcG93, table p. 129]). A representative of this orbit is obtained by chain cascade, by letting $\theta_{1}=\alpha_{1}+$ $2 \alpha_{2}+2 \alpha_{3}+3 \alpha_{4}+2 \alpha_{5}+\alpha_{6}$ be the highest root, $\theta_{2}=\alpha_{1}+\alpha_{3}+\alpha_{4}+\alpha_{5}+\alpha_{6}$, and $\theta_{3}=\alpha_{3}+\alpha_{4}+\alpha_{5}$. Then $\theta_{1}^{\vee}+\theta_{2}^{\vee}+\theta_{3}^{\vee}=-\varpi_{2}^{\vee}+\varpi_{3}^{\vee}+\varpi_{5}^{\vee}=s_{\alpha_{2}} s_{\alpha_{4}}\left(\varpi_{4}^{\vee}\right)$. Thus, the considered nilpotent orbit contains the element $e_{\theta_{1}^{\prime}}+e_{\theta_{2}^{\prime}}+e_{\theta_{3}^{\prime}}$ for $\theta_{1}^{\prime}, \theta_{2}^{\prime}, \theta_{3}^{\prime}$ defined by $\theta_{i}^{\prime}=s_{\alpha_{4}} s_{\alpha_{2}}\left(\theta_{i}\right)$. Here, the standard Levi subgroup $L$ corresponds to the root subsystem of type $A_{2} \times A_{2} \times A_{1}$ generated by $\alpha_{1}, \alpha_{2}, \alpha_{3}, \alpha_{5}, \alpha_{6}$.

As above, the involution $\sigma$ acts on the roots as the element $w$ of the Weyl group defined by $w=s_{\theta_{3}^{\prime}} \circ s_{\theta_{2}^{\prime}} \circ s_{\theta_{1}^{\prime}}=s_{\alpha_{4}} s_{\alpha_{2}} s_{\theta_{3}} s_{\theta_{2}} s_{\theta_{1}} s_{\alpha_{2}} s_{\alpha_{4}}$. By a straightforward computation, we see that $w\left(\alpha_{1}\right)=-\alpha_{6}, w\left(\alpha_{3}\right)=-\alpha_{5}$, and $w\left(\alpha_{2}\right)=\alpha_{2}$. Thus, $L^{\sigma}$ is a "diagonal" subgroup of $L$ of type $A_{2} \times A_{1}$.

In particular, in both examples, we see that the pair $\left(L, L^{\sigma}\right)$ fits the combinatorial setting described in Section 8

\section{Focus on nilpotent elements of Height 2}

We again consider the decomposition $Z=L_{Z} \ltimes U_{Z}$ of Proposition 1.1. In the previous section, we have shown that, whenever $e$ belongs to a spherical nilpotent orbit (which is equivalent to saying that $e$ is a nilpotent element of height at most 3; see Proposition (3.1), the subgroup $L_{Z}$ can be realized as a symmetric subgroup of the Levi subgroup $L$ (possibly up to certain connected components). In the present section, we focus on the unipotent subgroup $U_{Z}$. We point out the following fact:

Proposition 5.1. Assume that e is a nilpotent element of height 2 (i.e., $(\operatorname{ad} e)^{3}=$ $\left.0,(\operatorname{ad} e)^{2} \neq 0\right)$. Then $U_{Z}=U$, so that $Z=L_{Z} \ltimes U$ and $Z^{0}=L_{Z}^{0} \ltimes U$ in this case.

Proof. We consider the grading $\mathfrak{g}=\bigoplus_{i \in \mathbb{Z}} \mathfrak{g}(i)$ determined by $h$ (as in Section 1). From the representation theory of $\mathfrak{s l}_{2}$, we know that ad $e$ restricts to a map from $\mathfrak{g}(i)$ to $\mathfrak{g}(i+2)$ for every $i \in \mathbb{Z}$; moreover $(\operatorname{ad} e)^{i}$ induces a bijection between $\mathfrak{g}(-i)$ and $\mathfrak{g}(i)$ for every $i \geq 0$. The assumption that $e$ has height 2 implies that $\mathfrak{g}(i)=0$ whenever $|i|>2$, and we get the inclusion

$$
\operatorname{Lie}(U)=\bigoplus_{i>0} \mathfrak{g}(i)=\mathfrak{g}(1) \oplus \mathfrak{g}(2) \subset \mathfrak{z} \mathfrak{g}(e) .
$$

Since $U$ is connected, this yields $U \subset Z^{0}$, hence $U=U_{Z}$.

Corollary 5.2. Assume that $e$ is a nilpotent element of height 2. Then every $Z$ orbit $\mathcal{Z}$ of the flag variety $\mathcal{B}=G / B$ contains an element which is fixed by the torus $\tau\left(\mathbb{K}^{*}\right)$. Moreover, the subset of fixed points $\mathcal{Z}^{\tau}$ is a single $L_{Z}$-orbit. 
Proof. This fact follows from the more general assertion claimed in Lemma 7.1.

Remark 5.3.

(a) If a $Z$-orbit $\mathcal{Z} \subset \mathcal{B}$ contains elements fixed by $\tau$, then we have

$$
\lim _{t \rightarrow 0} \tau(t) \cdot p \in \mathcal{Z} \quad \text { for all } p \in \mathcal{Z} \text {. }
$$

Indeed, writing $p=(\ell u) \cdot p_{0}$ with $\ell \in L_{Z}, u \in U_{Z}, p_{0} \in \mathcal{Z}^{\tau}$, we get

$$
\tau(t) \cdot p=\ell\left(\tau(t) u \tau(t)^{-1}\right) \cdot p_{0} \rightarrow \ell \cdot p_{0} \in \mathcal{Z}^{\tau} \quad \text { as } t \rightarrow 0 .
$$

(b) When $e$ has height 3 , the conclusion of Corollary 5.2 is not valid in general, as shown by the following example. Let $\Phi^{+}=\left\{\epsilon_{i} \pm \epsilon_{j}: 1 \leq i<j \leq 3\right\} \cup\left\{\epsilon_{i}: 1 \leq\right.$ $i \leq 3\}$ be the usual system of positive roots of $G=\mathrm{SO}_{7}(\mathbb{K})$, and let $\alpha_{1}=\epsilon_{1}-\epsilon_{2}$, $\alpha_{2}=\epsilon_{2}-\epsilon_{3}, \alpha_{3}=\epsilon_{3}$ be the simple roots. Take nonzero root vectors $e_{\epsilon_{1}} \in \mathfrak{g}_{\epsilon_{1}}$ and $e_{\epsilon_{2}+\epsilon_{3}} \in \mathfrak{g}_{\epsilon_{2}+\epsilon_{3}}$ and let us consider the nilpotent element $e=e_{\epsilon_{1}}+e_{\epsilon_{2}+\epsilon_{3}}$. A cocharacter associated to $e$ is $\tau(t)=\epsilon_{1}^{\vee}+\left(\epsilon_{2}+\epsilon_{3}\right)^{\vee}=\varpi_{1}^{\vee}+\varpi_{3}^{\vee}$. Thus $e$ belongs to a spherical nilpotent orbit in view of Proposition 3.3 .

We consider the orthogonal form $\omega$ on $\mathbb{K}^{7}$ defined on the standard basis by

$$
\omega\left(\varepsilon_{i}, \varepsilon_{j}\right)= \begin{cases}1 & \text { if } i+j=8 \\ 0 & \text { otherwise }\end{cases}
$$

and we realize $G$ as the subgroup of elements $g \in \mathrm{SL}_{7}(\mathbb{K})$ which preserve this bilinear form. The flag variety $\mathcal{B}$ can be realized as the variety of isotropic flags

$$
\left\{\left(V_{1} \subset V_{2} \subset V_{3} \subset \mathbb{K}^{7}\right): \operatorname{dim} V_{i}=i, V_{3} \subset V_{3}^{\perp}\right\},
$$

where $\perp$ stands for the orthogonal with respect to $\omega$. We consider the flags $F=$ $\left(\left\langle\varepsilon_{1}+\varepsilon_{2}\right\rangle,\left\langle\varepsilon_{1}+\varepsilon_{2}, \varepsilon_{5}\right\rangle,\left\langle\varepsilon_{1}, \varepsilon_{2}, \varepsilon_{5}\right\rangle\right)$ and $F_{0}=\left(\left\langle\varepsilon_{2}\right\rangle,\left\langle\varepsilon_{2}, \varepsilon_{5}\right\rangle,\left\langle\varepsilon_{1}, \varepsilon_{2}, \varepsilon_{5}\right\rangle\right)$. Note that $\tau(t) \cdot F=\left(\left\langle t^{2} \varepsilon_{1}+t \varepsilon_{2}\right\rangle,\left\langle t^{2} \varepsilon_{1}+t \varepsilon_{2}, t^{-1} \varepsilon_{5}\right\rangle,\left\langle t^{2} \varepsilon_{1}, t \varepsilon_{2}, t^{-1} \varepsilon_{5}\right\rangle\right)$ for all $t \in \mathbb{K}^{*}$, so that $\lim _{t \rightarrow 0} \tau(t) \cdot F=F_{0}$. However, it can be computed that $\operatorname{dim} Z \cap \operatorname{Stab}_{G}(F)=5$ whereas $\operatorname{dim} Z \cap \operatorname{Stab}_{G}\left(F_{0}\right)=6$; hence $F$ and $F_{0}$ belong to distinct $Z$-orbits (of distinct dimensions). In view of part (a), this implies that the $Z$-orbit of $F$ contains no element fixed by $\tau$.

\section{Part 2. Structure of orbits on the flag variety for the action of certain spherical subgroups obtained through parabolic induction}

In this part of the paper, we assume that $H \subset G$ is a subgroup obtained through parabolic induction, i.e.,

$$
H=M \ltimes U,
$$

where

- $P \subset G$ is a parabolic subgroup and $P=L \ltimes U$ is a Levi decomposition ( $U$ is the unipotent radical, $L$ is a Levi factor of $P$ );

- $M \subset L$ is a spherical subgroup.

This is also the situation considered in [Br01, Lemma 7], where the weak Bruhat order of the $H$-orbits of the flag variety is described.

Although it is not assumed in this part, we are mostly interested in the case where $\left(L^{\sigma}\right)^{0} \subset M \subset L^{\sigma}$, for some involution $\sigma \in \operatorname{Aut}(L)$. For instance, Propositions 4.5 and 5.1 tell us that the role of $H$ can be played by the groups $Z$ and $Z^{0}$ where $Z:=Z_{G}(e)$ is the stabilizer of a nilpotent element of height 2 . 
The main goal of this part is to describe the structure of the orbits of $H$ on the flag variety. In this respect, our main result is Theorem 7.2 which shows that each $H$-orbit of $\mathcal{B}$ has a structure of algebraic affine bundle over an $M$-orbit of the flag variety $\mathcal{B}_{L}$ of the Levi subgroup $L$. This result is shown in Section 7 . Before that, in Section 6. we review some facts on the structure of parabolic orbits on the flag variety.

Let us mention that the structure of orbits for the action of a general spherical subgroup on the flag variety has been studied in [Br99, Proposition 2.2]: in particular, it is shown that each closed orbit is a flag variety for the spherical subgroup.

\section{Notation AND SHORT REVIEW ON PARABOLIC ORBITS}

We fix a maximal torus $T \subset G$. We also fix a Borel subgroup $B \subset G$ which contains $T$, and we consider the flag variety $\mathcal{B}=G / B$. Let $W$ be the Weyl group $N_{G}(T) / T$ of $G$. The choice of $B$ determines a set of simple reflections of $W$. Let $\ell(w)$ denote the length of an element $w \in W$ with respect to this set of simple reflections.

We consider a parabolic subgroup $P \subset G$ and a Levi decomposition $P=L \ltimes U$. There is no loss of generality in assuming that $B$ is contained in $P$ and $T$ is contained in $L$. Then, we can find a cocharacter $\tau: \mathbb{K}^{*} \rightarrow T$ such that $P, L, U$ are given by

$$
\left\{\begin{array}{l}
P=P(\tau):=\left\{g \in G: \lim _{t \rightarrow 0} \tau(t) g \tau(t)^{-1} \text { exists }\right\}, \\
U=U(\tau):=\left\{g \in G: \lim _{t \rightarrow 0} \tau(t) g \tau(t)^{-1}=1_{G}\right\}, \\
L=L(\tau):=\left\{g \in G: \tau(t) g \tau(t)^{-1}=g \quad \forall t \in \mathbb{K}^{*}\right\}=Z_{G}(\tau) .
\end{array}\right.
$$

Since $T \subset B \cap L$, the subgroup $B_{L}:=B \cap L$ is a Borel subgroup of $L$. We denote $\mathcal{B}_{L}=L / B_{L}$.

Let $W_{P}=N_{P}(T) / T$. Let ${ }^{P} W$ be a set of representatives of minimal length for the quotient $W_{P} \backslash W$.

We let $P$ act on $\mathcal{B}$, and it is well known that there are finitely many orbits for this action:

\section{Proposition 6.1.}

(a) $\mathcal{B}=\bigsqcup_{w \in P^{P}} \mathcal{P}_{w}$ where we denote $\mathcal{P}_{w}:=P w B / B$;

(b) $\operatorname{dim} \mathcal{P}_{w}=\ell(w)+\operatorname{dim} \mathcal{B}_{L}$ for all $w \in{ }^{P} W$.

In particular, each $P$-orbit $\mathcal{P}_{w}$ contains a fixed point of $T$ (namely $w B / B$ ), which is a fortiori fixed by the subtorus $\tau\left(\mathbb{K}^{*}\right)$. The next statement describes the structure of the orbits, by relying on the fixed point set of $\tau\left(\mathbb{K}^{*}\right)$.

Proposition 6.2. Let $\mathcal{B}^{\tau} \subset \mathcal{B}$ be the subvariety of fixed points of $\tau\left(\mathbb{K}^{*}\right)$. Let $\mathcal{P} \subset \mathcal{B}$ be a P-orbit. Let $\mathcal{P}^{\tau}=\mathcal{P} \cap \mathcal{B}^{\tau}$. Take any $p_{0}=g_{0} B \in \mathcal{P}^{\tau}$. Thus $g_{0} B g_{0}^{-1}$ is a Borel subgroup of $G$ which contains the torus $\tau\left(\mathbb{K}^{*}\right)$. This guarantees that $\left(g_{0} B g_{0}^{-1}\right) \cap L$ is a Borel subgroup of $L$.

(a) The map $L \rightarrow \mathcal{P}^{\tau}, \ell \mapsto \ell \cdot p_{0}$ is surjective and induces an isomorphism of varieties

$$
L /\left(g_{0} B g_{0}^{-1}\right) \cap L \rightarrow \mathcal{P}^{\tau} .
$$

In particular $\mathcal{P}^{\tau}$ is a projective variety, hence closed in $\mathcal{B}$. It is a connected component of $\mathcal{B}^{\tau}$ and every connected component of $\mathcal{B}^{\tau}$ is of this form. 
(b) There is a unique algebraic affine bundle $\phi_{\mathcal{P}}: \mathcal{P} \rightarrow \mathcal{P}^{\tau}$ such that

$$
\phi_{\mathcal{P}}\left(\ell u \cdot p_{0}\right)=\ell \cdot p_{0} \text { for all } \ell \in L \text {, all } u \in U \text {. }
$$

It does not depend on the choice of $p_{0} \in \mathcal{P}^{\tau}$.

In particular, part (a) of the Proposition says that the number of connected components of $\mathcal{B}^{\tau}$ is equal to the number of $\mathcal{P}$-orbits in $\mathcal{B}$, which is $\left|W / W_{P}\right|$.

Proof. The fact that $\left(g_{0} B g_{0}^{-1}\right) \cap L$ is a Borel subgroup of $L$ is Corollary 22.4 in Hu75. We first show (a). Let $p_{1} \in \mathcal{P}^{\tau}$. Thus $p_{0}, p_{1}$ both belong to $\mathcal{P}$; hence there is $g \in P$ such that $p_{1}=g \cdot p_{0}$. Moreover, we can write $g=\ell u$ with $\ell \in L$ and $u \in U$. Using (4), we have

$$
p_{1}=\tau(t) \cdot p_{1}=\tau(t) \ell u \cdot p_{0}=\tau(t) \ell u \cdot\left(\tau(t)^{-1} \cdot p_{0}\right)=\ell\left(\tau(t) u \tau(t)^{-1}\right) \cdot p_{0} \stackrel{t \rightarrow 0}{\longrightarrow} \ell \cdot p_{0},
$$

hence $p_{1}=\ell \cdot p_{0}$. We have shown that the map $L \rightarrow \mathcal{P}^{\tau}, \ell \mapsto \ell \cdot p_{0}$ is surjective. In addition, the isotropy group of $p_{0}\left(=g_{0} B\right)$ (in $L$ ) is clearly $L \cap\left(g_{0} B g_{0}^{-1}\right)$ and the fibers of this map are the cosets of $L \cap\left(g_{0} B g_{0}^{-1}\right)$. We deduce that the map $L \rightarrow \mathcal{P}^{\tau}$ induces an isomorphism of varieties $L /\left(g_{0} B g_{0}^{-1}\right) \cap L \stackrel{\sim}{\rightarrow} \mathcal{P}^{\tau}$. Since $\left(g_{0} B g_{0}^{-1}\right) \cap L$ is a Borel subgroup of $L$, we deduce that $\mathcal{P}^{\tau}$ is a projective variety. In particular it is closed in $\mathcal{B}$. Since $L$ is connected, it follows that the subsets $\mathcal{P}^{\tau}$ (attached to the various $P$-orbits $\mathcal{P}$ of $\mathcal{B}$ ) are closed, connected, pairwise disjoint, and they cover $\mathcal{B}^{\tau}$; hence they are exactly the connected components of $\mathcal{B}^{\tau}$. This shows (a).

Next we show (b). Consider the map

$$
\phi: \mathcal{B} \rightarrow \mathcal{B}^{\tau}, p \mapsto \lim _{t \rightarrow 0} \tau(t) \cdot p .
$$

It follows from Bialynicki-Birula's theorem Bi73. that the restriction of the map $\phi$ over each connected component of $\mathcal{B}^{\tau}$ is an algebraic affine bundle, i.e., $\left.\phi\right|_{\phi^{-1}\left(\mathcal{P}^{\tau}\right)}$ : $\phi^{-1}\left(\mathcal{P}^{\tau}\right) \rightarrow \mathcal{P}^{\tau}$ is an algebraic affine bundle for every $P$-orbit $\mathcal{P}$. Let $p \in \mathcal{P}$. We can write $p=\ell u \cdot p_{0}$ with $\ell \in L$ and $u \in U$. Using (4) and the fact that $p_{0}$ is fixed by $\tau$, we have

$$
\tau(t) \cdot p=\tau(t) \cdot\left(\ell u \cdot p_{0}\right)=\tau(t) \ell u \cdot\left(\tau(t)^{-1} \cdot p_{0}\right)=\ell\left(\tau(t) u \tau(t)^{-1}\right) \cdot p_{0} \stackrel{t \rightarrow 0}{\longrightarrow} \ell \cdot p_{0},
$$

hence

$$
\phi(p)=\phi\left(\ell u \cdot p_{0}\right)=\ell \cdot p_{0} \in \mathcal{P}^{\tau} .
$$

We get in particular $\phi(\mathcal{P}) \subset \mathcal{P}^{\tau}$, and this implies $\phi^{-1}\left(\mathcal{P}^{\tau}\right)=\mathcal{P}$. Hence $\phi$ restricts to an algebraic affine bundle $\phi_{\mathcal{P}}: \mathcal{P} \rightarrow \mathcal{P}^{\tau}$. Moreover the previous calculation shows that this map is such that $\phi_{\mathcal{P}}\left(\ell u \cdot p_{0}\right)=\ell \cdot p_{0}$ for all $(\ell, u) \in L \times U$, whenever $p_{0} \in \mathcal{P}^{\tau}$, and $\phi_{\mathcal{P}}$ is necessarily unique for satisfying the latter formula, because every element of $\mathcal{P}$ is of the form $\ell u \cdot p_{0}$.

The following observation will be useful.

Lemma 6.3. For every $w \in{ }^{P} W$, we have $\left(w B w^{-1}\right) \cap L=B_{L}$.

Proof. Since $w B w^{-1}$ is a Borel subgroup of $G$ which contains $T$, we know that $\left(w B w^{-1}\right) \cap L$ is a Borel subgroup of $L$. To show the lemma, it suffices to show that $B_{L} \subset\left(w B w^{-1}\right) \cap L$. Assume by contradiction $B_{L} \not \subset\left(w B w^{-1}\right) \cap L$. Since both subgroups are Borel subgroups of $L$ which contain the maximal torus $T$, it follows that there is a simple root $\alpha$ in the root system of $L$ such that $\mathfrak{g}_{\alpha}$ is not contained in $\operatorname{Lie}\left(w B w^{-1}\right)$. Hence $w^{-1}(\alpha)<0$. This implies $\ell\left(w^{-1} s_{\alpha}\right)=\ell\left(w^{-1}\right)-1$, 
hence $\ell\left(s_{\alpha} w\right)<\ell(w)$. Since $s_{\alpha} \in W_{P}$, this contradicts the assumption that $w$ is of minimal length among its coset $W_{P} w$.

Finally, the following statement summarizes the conclusions of Propositions 6.1 6.2 , it also uses Lemma 6.3 .

Proposition 6.4. Let $w \in{ }^{P} W$. We denote $\mathcal{P}_{w}^{\tau}=\mathcal{P}_{w} \cap \mathcal{B}^{\tau}$. Let $\phi_{w}=\phi_{\mathcal{P}_{w}}: \mathcal{P}_{w} \rightarrow$ $\mathcal{P}_{w}^{\tau}$ be the map defined in Proposition 6.2.

(a) We have $\mathcal{P}_{w}^{\tau}=L w B / B$. The map $L / B_{L} \rightarrow \mathcal{P}_{w}^{\tau}, \ell B_{L} \mapsto \ell w B / B$ is an isomorphism of algebraic varieties.

(b) The map $\phi_{w}: \mathcal{P}_{w} \rightarrow \mathcal{P}_{w}^{\tau}$ is an algebraic affine bundle whose typical fiber is the affine space of dimension $\ell(w)$.

\section{Parametrization and structure of $H$-Orbits}

We start with a preliminary observation.

Lemma 7.1. Let $H=M \ltimes U$ be as in (3). Then, every $H$-orbit $\mathcal{H}$ of the flag variety $\mathcal{B}=G / B$ contains an element which is fixed by the torus $\tau\left(\mathbb{K}^{*}\right)$. Moreover, the subset of fixed points $\mathcal{H}^{\tau}$ is a single $M$-orbit.

Proof. Since $H \subset P$, the orbit $\mathcal{H}$ is contained in some $P$-orbit $\mathcal{P}$. As recalled in the previous subsection, the subset of fixed points $\mathcal{P}^{\tau}$ is nonempty, so let $p_{0} \in \mathcal{P}^{\tau}$. There is $g \in P$ such that $g \cdot p_{0} \in \mathcal{H}$. We write $g=u \ell$ with $\ell \in L$ and $u \in U$. By assumption, $U$ is contained in $H$, so $u \in H$, hence $\ell \cdot p_{0}=u^{-1} \cdot\left(g \cdot p_{0}\right) \in \mathcal{H}$. In addition since $p_{0}$ is fixed by the torus $\tau\left(\mathbb{K}^{*}\right)$ and $\ell(\in L)$ commutes with every $\tau(t)$ $\left(t \in \mathbb{K}^{*}\right)$, we get that $\ell \cdot p_{0}$ is also fixed by $\tau\left(\mathbb{K}^{*}\right)$. Hence $\mathcal{H}^{\tau} \neq \emptyset$.

Now let $p_{0}, p_{1} \in \mathcal{H}^{\tau}$. There is $g \in H$ such that $p_{1}=g \cdot p_{0}$. Writing $g=\ell u$ with $\ell \in M$ and $u \in U$, we have for every $t \in \mathbb{K}^{*}$ that

$$
p_{1}=\tau(t) \cdot p_{1}=\ell\left(\tau(t) u \tau(t)^{-1}\right) \cdot p_{0},
$$

hence, by passing to the limit as $t \rightarrow 0$, we obtain $p_{1}=\ell \cdot p_{0}$.

By assumption $M$ is a spherical subgroup of $L$; hence the action of $M$ on the flag variety $\mathcal{B}_{L}=L / B_{L}$ has finitely many orbits. Let $\Xi \subset L$ be a set of representatives of the orbits, i.e., giving rise to the decomposition

$$
\mathcal{B}_{L}=\bigsqcup_{\xi \in \Xi} \mathcal{M}_{L}(\xi) \quad \text { where } \quad \mathcal{M}_{L}(\xi):=M \xi B_{L} / B_{L}
$$

Let $d(\xi)=\operatorname{dim} \mathcal{M}_{L}(\xi)$.

Theorem 7.2. For every $(w, \xi) \in{ }^{P} W \times \Xi$, we set $\mathcal{H}_{w, \xi}:=H \xi w B / B$. (This is an $H$-orbit of $\mathcal{B}$.)

(a) Every $H$-orbit of $\mathcal{B}$ is of the form $\mathcal{H}_{w, \xi}$ for a unique pair $(w, \xi) \in{ }^{P} W \times \Xi$.

(b) The $\tau$-fixed point set $\mathcal{H}_{w, \xi}^{\tau}$ is given by $\mathcal{H}_{w, \xi}^{\tau}=M \xi w B / B$ and it is ( $M$ equivariantly) isomorphic to $\mathcal{M}_{L}(\xi)$.

(c) The affine bundle $\phi_{w}: \mathcal{P}_{w} \rightarrow \mathcal{P}_{w}^{\tau}$ of Proposition 6.4(b) satisfies $\phi_{w}^{-1}\left(\mathcal{H}_{w, \xi}^{\tau}\right)$ $=\mathcal{H}_{w, \xi}$; hence it restricts to an algebraic affine bundle

$$
\mathcal{H}_{w, \xi} \rightarrow \mathcal{H}_{w, \xi}^{\tau} \cong \mathcal{M}_{L}(\xi)
$$

of fiber isomorphic to $\mathbb{A}^{\ell(w)}$. In particular, $\operatorname{dim} \mathcal{H}_{w, \xi}=\ell(w)+d(\xi)$. 
Proof. Since $H \subset P$, we have a partition

$$
\mathcal{B} / H=\bigsqcup_{w \in \in^{P} W} \mathcal{P}_{w} / H
$$

Let $w \in{ }^{P} W$ and let us consider the $P$-orbit $\mathcal{P}_{w}$ and its set of $H$-orbits. We also consider the fixed point set $\mathcal{P}_{w}^{\tau}$ and its set of $M$-orbits. It follows from Lemma 7.1 that the map

$$
\mathcal{P}_{w} / H \rightarrow \mathcal{P}_{w}^{\tau} / M, \mathcal{H} \mapsto \mathcal{H}^{\tau}
$$

is well defined; in addition this map is clearly injective and surjective, hence bijective. Let us analyze the decomposition of the subvariety $\mathcal{P}_{w}^{\tau}$ into $M$-orbits. As noted in Proposition 6.4, we have an isomorphism

$$
\mathcal{B}_{L}=L / B_{L} \rightarrow \mathcal{P}_{w}^{\tau}, \ell B_{L} \mapsto \ell w B / B .
$$

Therefore, the decomposition $\mathcal{B}_{L}=\bigsqcup_{\xi \in \Xi} \mathcal{M}_{L}(\xi)$, with $\mathcal{M}_{L}(\xi)=M \xi B_{L} / B_{L}$, induces the decomposition into $M$-orbits

$$
\mathcal{P}_{w}^{\tau}=\bigsqcup_{\xi \in \Xi} M \xi w B / B, \quad \text { and } \quad \mathcal{M}_{L}(\xi) \cong M \xi w B / B .
$$

The definition of $\mathcal{H}_{w, \xi}$ implies that $\mathcal{H}_{w, \xi}$ is the unique $H$-orbit of $\mathcal{P}_{w}$ such that $\mathcal{H}_{w, \xi}^{\tau}=M \xi w B / B$. This shows parts (a) and (b).

In order to show part (c), it suffices to show the inclusion $\phi_{w}(\mathcal{H}) \subset \mathcal{H}^{\tau}$ for every $H$-orbit $\mathcal{H} \subset \mathcal{P}_{w}$. Then, knowing that the $H$-orbits are pairwise disjoint, this forces $\phi_{w}^{-1}\left(\mathcal{H}_{w, \xi}^{\tau}\right)=\mathcal{H}_{w, \xi}$, and the properties of the restriction $\left.\phi_{w}\right|_{\mathcal{H}_{w, \xi}}$ are inherited from the properties of $\phi_{w}$ stated in Proposition 6.4(b). So let $\mathcal{H} \subset \mathcal{P}_{w}$ be an $H$-orbit. Take any $p_{0} \in \mathcal{H}^{\tau}$. Thus $\mathcal{H}=\left\{\ell u \cdot p_{0}: \ell \in M, u \in U\right\}$, and by the characterization of $\phi_{w}=\phi_{\mathcal{P}_{w}}$ given in Proposition [6.2, we have $\phi_{w}\left(\ell u \cdot p_{0}\right)=\ell \cdot p_{0} \in \mathcal{H}^{\tau}$ for all $\ell u \cdot p_{0} \in \mathcal{H}$. This shows part (c) of the statement.

We summarize the obtained results about $H$-orbits with the following diagram:

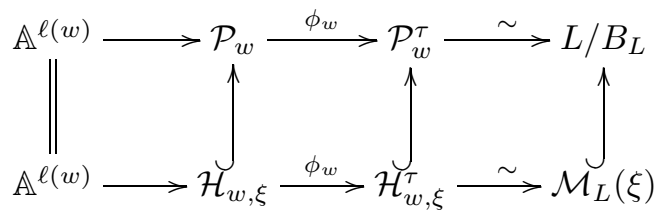

Example 7.3. Let $G=\mathrm{GL}_{n}(\mathbb{K})$ and let $H=Z:=Z_{G}(e)$ be the stabilizer of a nilpotent matrix $e \in \mathfrak{g l}_{n}(\mathbb{K})$ such that $e^{2}=0$. Let $r=$ rank $e$. As shown in Example 4.7 and Proposition 5.1, the parabolic subgroup $P=L \ltimes U$ associated to $e$ has a Levi factor of the form $L=\mathrm{GL}_{r}(\mathbb{K}) \times \mathrm{GL}_{r}(\mathbb{K}) \times \mathrm{GL}_{n-2 r}(\mathbb{K})$, while $Z$ is of the form $L_{Z} \ltimes U$ with $L_{Z}=\Delta \mathrm{GL}_{r}(\mathbb{K}) \times \mathrm{GL}_{n-2 r}(\mathbb{K})$. (Here $\Delta \mathrm{GL}_{r}(\mathbb{K})$ stands for the diagonal embedding of $\mathrm{GL}_{r}(\mathbb{K})$ into $\mathrm{GL}_{r}(\mathbb{K}) \times \mathrm{GL}_{r}(\mathbb{K})$.)

In this example:

- ${ }^{P} W$ is the set of minimal length representatives of the quotient $\left(\mathfrak{S}_{r} \times \mathfrak{S}_{r} \times\right.$ $\left.\mathfrak{S}_{n-2 r}\right) \backslash \mathfrak{S}_{n}$

- the flag variety $\mathcal{B}_{L}$ is a triple flag variety isomorphic to $\mathcal{B}_{r} \times \mathcal{B}_{r} \times \mathcal{B}_{n-2 r}$, where $\mathcal{B}_{k}$ stands for the flag variety $\mathrm{GL}_{k}(\mathbb{K}) / B_{k}$ of $\mathrm{GL}_{k}(\mathbb{K})$;

- the $L_{Z}$-orbits of $\mathcal{B}_{L}$ are parametrized by the permutations $v \in \mathfrak{S}_{r}$ and take the form $\mathbb{O}_{v} \times \mathcal{B}_{n-2 r}$, where $\mathbb{O}_{v}=\mathrm{GL}_{r}(\mathbb{K}) \cdot\left(v B_{r}, B_{r}\right) \cong \mathrm{GL}_{r}(\mathbb{K}) \times{ }^{B_{r}} \mathbb{A}^{\ell(v)}$. 
Therefore, in this example, the $Z$-orbits of $\mathcal{B}$ are parametrized by the pairs $(w, v) \in$ ${ }^{P} W \times \mathfrak{S}_{r}$, and each orbit is an algebraic affine bundle over $\mathcal{B}_{r} \times \mathcal{B}_{n-2 r}$ of fiber isomorphic to the affine space $\mathbb{A}^{\ell(w)} \times \mathbb{A}^{\ell(v)}$. In particular, since the double flag variety $\mathcal{B}_{r} \times \mathcal{B}_{n-2 r}$ has a natural cell decomposition (the product of the Schubert cell decompositions of $\mathcal{B}_{r}$ and $\mathcal{B}_{n-2 r}$ ), we deduce that each $Z$-orbit of $\mathcal{B}$ has a cell decomposition; moreover, the number of cells and the codimensions of the cells are the same for each $Z$-orbit.

\section{Part 3. Bruhat order}

In this part, our motivation is to understand the (strong) Bruhat order for the orbits of $Z=Z_{G}(e)$ on the flag variety $\mathcal{B}=G / B$, or equivalently for the orbits of $B$ on the nilpotent orbit $G \cdot e=G / Z$, where $e$ is nilpotent element of height 2. In type $A$, the order is described by Boos and Reineke BR12] in terms of link patterns. Their approach is based on representations of quivers.

Actually, our motivation is (more generally) to have a description of the (strong) Bruhat order for the orbits of a subgroup $H$ of the form

$$
H=M \ltimes U,
$$

where $P=L U$ is the Levi decomposition of a parabolic subgroup and $\left(L^{\sigma}\right)^{0} \subset$ $M \subset L^{\sigma}$ for some involution $\sigma \in \operatorname{Aut}(L)$.

Note that if $\sigma=\mathrm{id}_{L}$, then $H$ is a parabolic subgroup and the strong order is in this case the Bruhat order on the parabolic quotient ${ }^{P} W$ of the Weyl group. If $P=G$, then $H$ is a symmetric subgroup of $G$ (possibly up to certain connected components) and the strong order is described by Richardson and Springer [RS90, RS92. If $e$ is a nilpotent element of height 2, then its stabilizer $Z$ (as well as the connected subgroup $Z^{0}$ ) is of the form considered in (5) (see Propositions 4.5] and 5.1). Note also that the description of the weak Bruhat order for $H$ as in (5) (or even more generally for $H$ obtained through parabolic induction from a spherical subgroup of $L)$ is given in $\mathrm{Br} 01$.

In this part, we propose a combinatorial model which reflects the geometric situation of (5). We first introduce a Coxeter-theoretic partial order, defined by using a parabolic subgroup of a Coxeter group $W$ which is equipped with an involution (Section 8 ). In type $A$, this order coincides with the order given by inclusions of the $Z_{G}(e)$-orbit closures (for a spherical nilpotent orbit $G \cdot e$ ), and we give additional combinatorial criteria in this case (Section 9). We also give necessary and sufficient conditions for a relation to be a cover relation (see Theorem 8.16); in [BR12, necessary conditions for a relation to be a cover relation are given, but these conditions are not sufficient in general.

The notation used in Part 3 is independent of the notation used in Parts 12

\section{A CoXeter-theoretic PARTial order}

Let $(W, S)$ be a Coxeter system (with $S$ finite, but $W$ may be infinite). We denote by $\leq$ the (strong) Bruhat order on $W$. Recall that for $u, v \in W$, we have $u \leq v$ if and only if every reduced expression of $v$ has a subword which is a reduced expression of $u$ (see [De77]). Also recall that every subset $L \subset S$ induces a decomposition

$$
W=W^{L} W_{L} \cong W^{L} \times W_{L},
$$


where $W_{L} \subset W$ is the subgroup generated by $L$ and $W^{L}:=\{w \in W: \ell(w s)>$ $\ell(w) \forall s \in L\}$.

Let $I, J, K \subset S$ satisfy the following two conditions:

(1) There is an isomorphism $\sigma: W_{I} \longrightarrow W_{J}, x \mapsto x^{*}$ of Coxeter groups,

(2) $W_{I \cup J \cup K}=W_{I} \times W_{J} \times W_{K}$.

It follows in particular from the first point that $x \mapsto x^{*}$ preserves the Bruhat order, that is, we have $x \leq y$ if and only if $x^{*} \leq y^{*}$. The second point means that the subsets $I, J, K$ are disjoint and disconnected. Note that $W_{I} \rightarrow W, x \mapsto x x^{*}$ is a monomorphism of groups; we denote by $W_{I, J}$ its image:

$$
W_{I, J}=\left\{x x^{*}: x \in W_{I}\right\} .
$$

We have $W_{I} \cap W_{I, J}=\{1\}$ and

$$
W_{I} \times W_{J}=W_{I \cup J}=W_{I} W_{I, J}=W_{J} W_{I, J} .
$$

Using the decomposition $W=W^{I \cup J \cup K} W_{I \cup J \cup K}$, we get that every $w \in W$ can be written uniquely as a product $x_{1} x_{2} x_{3} x x^{*}$ with $x_{1} \in W^{I \cup J \cup K}, x_{2}, x \in W_{I}, x_{3} \in W_{K}$. Note that

$$
\ell(w)=\ell\left(x_{1}\right)+\ell\left(x_{2} x x^{*}\right)+\ell\left(x_{3}\right)=\ell\left(x_{1}\right)+\ell\left(x_{2} x\right)+\ell(x)+\ell\left(x_{3}\right) .
$$

We set $W(I, J, K)=W^{I \cup J \cup K} W_{I}=W^{J \cup K}$. Note that it gives a set of representatives of the left cosets of the subgroup $W_{K} W_{I, J} \subset W$. The following order defined on this quotient will in fact not depend on this particular choice of representatives in view of Remark 8.5(b).

Given $w \in W$, we set $[w]=w W_{K} W_{I, J}$. In this section, our aim is to study the left cosets $[w]$, the set of representatives $W(I, J, K)$, and the following binary relation:

For $w, w^{\prime} \in W(I, J, K)$, we write $w^{\prime} \leq_{\mathcal{O}} w$ if there is $u \in\left[w^{\prime}\right]$ such that $u \leq w$.

Our first claim is:

Proposition 8.1. The relation $\leq_{\mathcal{O}}$ defines a partial order on $W(I, J, K)$.

This assertion is shown in Section 8.1, where preliminary remarks are also made. In Section 8.2 we describe the elements of minimal length $\ell$ in each left coset: it turns out that there can be several elements of minimal length in a coset. We show that the definition of the order $\leq_{\mathcal{O}}$ can be rephrased in terms of these minimal elements (Corollary 8.10). In Section 8.3, we characterize the cover relations for $\leq_{\mathcal{O}}$, and our final conclusion is that the poset $\left(W(I, J, K), \leq_{\mathcal{O}}\right)$ is graded with rank function given by the restriction of the length $\ell$ to $W(I, J, K)$ (Corollary 8.17).

8.1. Partial order $\leq_{\mathcal{O}}$. The proof of Proposition 8.1 is based on Lemma 8.2,

Lemma 8.2. Let $w, w_{1} \in W(I, J, K)$ and assume that $w \leq_{\mathcal{O}} w_{1}$. Then for every $w_{2} \in\left[w_{1}\right]$, there is $v \in[w]$ such that $v \leq w_{2}$.

To prove this lemma, we need:

Lemma 8.3. Let $x, y \in W$. Let $x^{\prime} \leq x$. There is $y^{\prime} \leq y$ such that $x^{\prime} y^{\prime} \leq x y$.

Proof of Lemma 8.3. We argue by induction on $\ell(y)$. If $\ell(y)=0$, then the claim holds with $y^{\prime}=1$. If $\ell(y)=1$, then $y=s \in S$. If $x s>x$, then the claim holds with $y^{\prime}=1$. Hence assume that $x s<x$. We choose a reduced expression $s_{1} s_{2} \cdots s_{k}$ of $x$ with $s=s_{k}$. Since $x^{\prime} \leq x$ we have that $x^{\prime}$ has a reduced expression 
occurring as a subword of $s_{1} s_{2} \cdots s_{k}$. If $s_{k}$ does not contribute to that subword, then $x^{\prime} \leq x s=x y$ and we are done with $y^{\prime}=1$. Hence assume that $s_{k}=s$ contributes to the subword giving a reduced expression of $x^{\prime}$. Deleting the last letter gives a subword of $s_{1} s_{2} \cdots s_{k-1}=x s=x y$; hence with $y^{\prime}=s$ we get the claim.

Now assume that $\ell(y)>1$. Let $s \in S$ be such that $y s<y$. By induction on length, there is $u \leq y s$ such that $x^{\prime} u \leq x y s$. By the case of length one, there is $v \leq s$ such that $x^{\prime} u v \leq x y$. Since $y s<y$, we have that $y^{\prime}:=u v$ has a (not necessarily reduced) expression which occurs as a subword of a reduced expression of $y$. Since every expression for an element of a Coxeter group has a reduced expression for it as a subword, we have $y^{\prime} \leq y$, and we have already seen that $x^{\prime} y^{\prime} \leq x y$.

Proof of Lemma 8.2. By assumption, there is $u \in[w]$ such that $u \leq w_{1}$. Write $w_{2}=w_{1} a x x^{*}$ with $x \in W_{I}, a \in W_{K}$. It suffices to show that there are $y \in W_{I}$, $b \in W_{K}$ such that $u b y y^{*} \leq w_{1} a x x^{*}$.

Since $u \leq w_{1}$, Lemma 8.3 yields an element $b \leq a$ such that $u b \leq w_{1} a$. By Lemma 8.3 again, there is $y \leq x$ such that $u b y \leq w_{1} a x$. Note that $x \in W_{I}$ and $a \in W_{K}$, hence $y \in W_{I}$ and $b \in W_{K}$, because $W_{I}$ and $W_{K}$ are parabolic. Now since $w_{1} a x \in W(I, J, K) W_{K} W_{I}$, we have that $\ell\left(w_{1} a x x^{*}\right)=\ell\left(w_{1} a x\right)+\ell\left(x^{*}\right)$. But $y \leq x$ implies that $y^{*} \leq x^{*}$; hence ubyy* has a (not necessarily reduced) expression which is a subword of a reduced expression of $w_{1} a x x^{*}$. It follows that $v:=u b y y^{*} \leq w_{1} a x x^{*}=w_{2}$, which is what we wanted to show.

Proof of Proposition 8.1. Reflexivity is clear. We show antisymmetry. If $x, y \in$ $W(I, J, K)$ satisfy $x \leq_{\mathcal{O}} y$ and $y \leq_{\mathcal{O}} x$, then since elements of $W(I, J, K)$ have minimal length in their cosets modulo $W_{K} W_{I, J}$, we get that $\ell(x)=\ell(y)$; as there is $u \in[y]$ such that $u \leq x$, this forces $u=x$, hence $[x]=[y]$, and thus $x=y$.

We now show transitivity. Let $z \leq_{\mathcal{O}} y \leq_{\mathcal{O}} x$. By definition of the relation $\leq_{\mathcal{O}}$, there is $w \in[y]$ such that $w \leq x$. By Lemma 8.2, there is $w^{\prime} \in[z]$ such that $w^{\prime} \leq w$. We then have $w^{\prime} \leq x$ with $w^{\prime} \in[z]$, which is precisely the definition of $z \leq_{\mathcal{O}} x$.

Example 8.4. In type $A_{3}$ with $I=\left\{s_{1}\right\}, J=\left\{s_{3}\right\}, K=\emptyset$, the order $\leq_{\mathcal{O}}$ on $W(I, J, K)$ is given in Figure 1 .

\section{Remark 8.5.}

(a) Note that $W(I, J, K)=W^{J \cup K}$, and $W^{J \cup K}$ is naturally ordered by the restriction of the Bruhat order on $W$. The orders $\leq$ and $\leq_{\mathcal{O}}$ on $W(I, J, K)$ do not coincide in general. For instance, taking $W, I, J$, and $\bar{K}$ as in Example 8.4, we have $s_{1} \leq_{\mathcal{O}} s_{3} s_{2}$, while $s_{1} \not \leq s_{3} s_{2}$.

(b) As Lemma 8.2 shows, the partial order $\leq_{\mathcal{O}}$ is also defined on the quotient $W /\left(W_{K} W_{I, J}\right) \simeq W(I, J, K)$ by the equivalence $[w] \leq_{\mathcal{O}}\left[w^{\prime}\right]$ if and only if for every $u^{\prime} \in\left[w^{\prime}\right]$, there is $u \in[w]$ such that $u \leq u^{\prime}$.

(c) Let $(W, S)$ be a Coxeter system, $L \subset S$ and $W_{L}$ the standard parabolic subgroup generated by $L$. Then $\left(W_{L}, L\right)$ is a Coxeter system. Let $\theta$ be an automorphism of the Dynkin diagram of $L$; it extends to an automorphism of the Coxeter group $\left(W_{L}, L\right)$, which we also denote by $\theta$. Let $W_{L}^{\theta}$ be the subgroup of $\theta$-fixed points of $W_{L} \subset W$. We can generalize the partial order $\leq_{\mathcal{O}}$ to $W / W_{L}^{\theta}$ as follows: for $u, v \in W$, we have $u W_{L}^{\theta} \leq_{\mathcal{O}} v W_{L}^{\theta}$ if and only if for every $w \in v W_{L}^{\theta}$, there is $w^{\prime} \in u W_{L}^{\theta}$ such that $w^{\prime} \leq w$. In the case described above, we have $L=I \cup K \cup J$, and the automorphism $\theta$ of $W_{L}=W_{I} \times W_{K} \times W_{J}$ is given by $\left(x, y, z^{*}\right) \mapsto\left(z, y, x^{*}\right)$. 


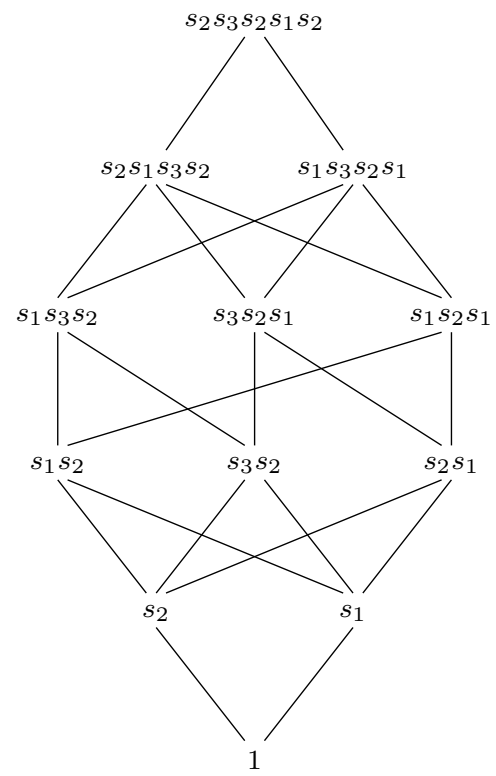

Figure 1. The order $\leq_{\mathcal{O}}$ on $W(I, J, K)$ in type $A_{3}$ with $I=\left\{s_{1}\right\}$, $J=\left\{s_{3}\right\}, K=\emptyset$

We have $W_{L}^{\theta}=W_{K} W_{I, J}$. The fact that the two partial orders coincide follows from Lemma 8.2 (or from part (b) of this remark).

8.2. Elements of minimal length in $[w]$. Given $w \in W(I, J, K)$, the left coset $[w]$ does not have a single representative of minimal length in general. But $w$ has minimal length in $[w]$ : indeed, writing $w=w_{1} w_{2}$ with $w_{1} \in W^{I \cup J \cup K}, w_{2} \in W_{I}$, then for any $x \in W_{I}, a \in W_{K}$ and $w^{\prime}=w_{1} w_{2} a x x^{*}$ we have that

$$
\ell\left(w^{\prime}\right)=\ell\left(w_{1}\right)+\ell\left(w_{2} x\right)+\ell\left(x^{*}\right)+\ell(a)=\ell\left(w_{1}\right)+\ell\left(w_{2} x\right)+\ell(x)+\ell(a),
$$

and since $\ell\left(w_{2} x\right)+\ell(x) \geq \ell\left(w_{2}\right)$ we get that $\ell\left(w^{\prime}\right) \geq \ell(w)$. Moreover, every element of minimal length in $[w]$ lies in $W^{K}$, but the converse does not hold in general.

For $w \in W(I, J, K)$, let

$$
\operatorname{Min}(w)=\{u \in[w]: \ell(u)=\ell(w)\}
$$

be the set of elements of minimal length in the coset $[w]$. On the basis of the above calculation, we have the following easy results:

Lemma 8.6. Let $w=w_{1} w_{2} \in W(I, J, K)$ with $w_{1} \in W^{I \cup J \cup K}$ and $w_{2} \in W_{I}$.

(a) $\operatorname{Min}(w)=\left\{w x x^{*}: x \in W_{I}, \ell\left(w_{2} x\right)+\ell\left(x^{-1}\right)=\ell\left(w_{2}\right)\right\}$

(b) $\operatorname{Min}(w)=w_{1} \cdot \operatorname{Min}\left(w_{2}\right)$.

In particular, the set $\operatorname{Min}(w)$ is in bijection with the set of elements lying below $w_{2}$ for the right weak order, and we have $|\operatorname{Min}(w)|=1$ iff $w_{2}=1$ iff $w \in W^{I \cup J \cup K}$.

Lemma 8.7. Let $w \in W(I, J, K), v \in[w]$. There is $u \in \operatorname{Min}(w)$ such that $u \leq v$. In other words, $\operatorname{Min}(w)$ is also characterized as being the set of elements in $[w]$ which are minimal for the Bruhat order. 
Proof. Let $w=w_{1} w_{2}$ be as above. Let $v=w_{1} w_{2} a x x^{*}$, with $a \in W_{K}, x \in W_{I}$. Note that $v^{\prime}:=w_{1} w_{2} x x^{*} \leq v$ since $v=v^{\prime} a$ and $\ell(v)=\ell\left(v^{\prime}\right)+\ell(a)$. If $v^{\prime} \in \operatorname{Min}(w)$, then we are done. Assume that this is not the case: we then claim that there is $y \in W_{I}$ such that $w_{1} w_{2} y y^{*}<w_{1} w_{2} x x^{*}$, which is enough to conclude, since we can then iterate until reaching an element of minimal length.

To show the claim, let $s_{1} s_{2} \cdots s_{k}$ be a reduced expression of $w_{2} x$, and $s_{k+1} \cdots s_{m}$ be a reduced expression of $x^{-1}$. We have $\ell\left(w_{2} x x^{*}\right)=\ell\left(w_{2} x\right)+\ell\left(x^{*}\right)=m$ since $I$ and $J$ are disconnected.

Since we assume that $w_{1} w_{2} x x^{*} \notin \operatorname{Min}(w)$, we have $\ell\left(w_{2} x\right)+\ell\left(x^{-1}\right)>\ell\left(w_{2}\right)$ (by Lemma [8.6); hence the expression $s_{1} s_{2} \cdots s_{m}$ is not reduced. Since both $s_{1} s_{2} \cdots s_{k}$ and $s_{k+1} \cdots s_{m}$ are reduced, there exists a minimal $i \in\{k+1, \ldots, m\}$ such that $s_{1} s_{2} \cdots s_{i}$ is not reduced, and by the exchange lemma we have $s_{1} s_{2} \cdots s_{i}=$ $s_{1} s_{2} \cdots \widehat{s_{j}} \cdots s_{i-1}$ for some $j \in\{1, \ldots, k\}$, where the notation $\widehat{s_{j}}$ indicates that $s_{j}$ is removed. It follows that

$$
z^{\prime}:=w_{1} w_{2} x x^{*}\left(s_{k+1} \cdots s_{i}\right)\left(s_{k+1}^{*} \cdots s_{i}^{*}\right)=w_{1}\left(s_{1} \cdots \widehat{s}_{j} \cdots s_{i-1}\right)\left(s_{m}^{*} s_{m-1}^{*} \cdots s_{i+1}^{*}\right) .
$$

Multiplying $z^{\prime}$ on the right by $\left(s_{i-1} s_{i-2} \cdots s_{k+1}\right)\left(s_{i-1}^{*} s_{i-2}^{*} \cdots s_{k+1}^{*}\right)$ we get an element

$$
z:=w_{1}\left(s_{1} \cdots \widehat{s_{j}} \cdots s_{k}\right)\left(s_{m}^{*} s_{m-1}^{*} \cdots \widehat{s_{i}^{*}} \cdots s_{k+1}^{*}\right) \in[w] .
$$

We have $\left(s_{1} \cdots \widehat{s_{j}} \cdots s_{k}\right)<w_{2} x=s_{1} \cdots s_{k}$ and $\left(s_{m}^{*} s_{m-1}^{*} \cdots \widehat{s_{i}^{*}} \cdots s_{k+1}^{*}\right)<x^{*}=$ $s_{m}^{*} \cdots s_{k+1}^{*}$; hence since $w_{1} \in W^{I \cup J \cup K}, w_{2} x \in W_{I}$ and $x^{*} \in W_{J}$, we get $z<$ $w_{1} w_{2} x x^{*}$. Since $z=w_{1} w_{2} y y^{*}$ where $y=x\left(s_{k+1} s_{k+2} \cdots s_{i} s_{i-1} \cdots s_{k+1}\right)$, the proof is complete.

If $\theta$ is an automorphism of a Coxeter group $(W, S)$ induced by an automorphism of the Dynkin diagram, and if each orbit of $\theta$ in $S$ generates a finite group, then it is well known that the subgroup of fixed points $W^{\theta}$ is again a Coxeter group (see for instance $\mathrm{Mu} 93$ ]). Moreover, the canonical generators of $W^{\theta}$ are obtained as follows. For each orbit of $\theta$ on the simple system $S$, consider the standard parabolic subgroup generated by the simple reflections in this orbit. Take the longest element in this subgroup (which is finite by assumption). Then the set of all such longest elements, for all orbits, forms the simple system of the subgroup of fixed points.

In our case, considering the automorphism $\theta$ of $L=I \cup J \cup K$ as in Remark 8.5)(c), we have $W_{L}^{\theta}=W_{K} W_{I, J}$; the orbits of $\theta$ are of the form $\{s\}$ for $s \in K$ or $\left\{s, s^{*}\right\}$ for $s \in I$. Hence the corresponding generators of $W_{K} W_{I, J}$ viewed as a Coxeter group are given by $\{s: s \in K\} \cup\left\{s s^{*}: s \in I\right\}$. We write $\Theta=\left\{s s^{*}: s \in I\right\}$.

Lemma 8.8. Let $u, u^{\prime} \in \operatorname{Min}(w)$. There is a sequence

$$
u, u x_{1}, u x_{1} x_{2}, \ldots, u x_{1} x_{2} \cdots x_{k}=u^{\prime}
$$

with $x_{1}, x_{2}, \ldots, x_{k} \in \Theta$ such that for all $i, u x_{1} x_{2} \cdots x_{i} \in \operatorname{Min}(w)$. In other words, any two elements in $\operatorname{Min}(w)$ can be related by multiplying on the right by a sequence of generators of $W_{K} W_{I, J}$ coming only from $\Theta$, and such that at each step, the obtained element still has minimal length in $[w]$.

Proof. Since the elements of $\Theta$ have order two, it suffices to show the claim for $u^{\prime}=$ $w$. Let $w=w_{1} w_{2}$ be as above. By Lemma 8.6, there is $x \in W_{I}$ such that $\ell\left(w_{2} x\right)+$ $\ell\left(x^{-1}\right)=\ell\left(w_{2}\right)$ and $u=w x x^{*}=w_{1} w_{2} x x^{*}$. Let $x=s_{k} s_{k-1} \cdots s_{2} s_{1}$ be a reduced expression of $x$. Setting $x_{i}=s_{i} s_{i}^{*}$ and using the fact that $I$ and $J$ are disconnected 
and that $\ell\left(w_{2} x\right)+\ell\left(x^{-1}\right)=\ell\left(w_{2}\right)$ (hence that $w_{2}$ has a reduced expression ending by $\left.x^{-1}\right)$, we obtain $u x_{1} x_{2} \cdots x_{i}=w_{1} w_{2} s_{k} s_{k-1} \cdots s_{i+1} s_{k}^{*} s_{k-1}^{*} \cdots s_{i+1}^{*}$ and

$$
\begin{aligned}
\ell\left(u x_{1} x_{2} \cdots x_{i}\right) & =\ell\left(w_{1}\right)+\ell\left(w_{2} s_{k} s_{k-1} \cdots s_{i+1}\right)+\ell\left(s_{k}^{*} s_{k-1}^{*} \cdots s_{i+1}^{*}\right) \\
& =\ell\left(w_{1}\right)+\ell\left(w_{2}\right)-(k-i)+(k-i)=\ell\left(w_{1} w_{2}\right)=\ell(w),
\end{aligned}
$$

which concludes the proof.

Lemma 8.9. Let $w \in W(I, J, K)$. Let $u \in \operatorname{Min}(w), u^{\prime} \in W$ and $s \in I$. Assume that $u^{\prime}<u$ and $\ell\left(u s s^{*}\right)=\ell(u)$. Then there exists $y$ in $\left\{u^{\prime}, u^{\prime} s s^{*}\right\} \subset\left[u^{\prime}\right]$ such that $\ell(y) \leq \ell\left(u^{\prime}\right)$ and $y<$ uss $^{*}$. In particular, if $u^{\prime} \in \operatorname{Min}\left(w^{\prime}\right)$ for some $w^{\prime} \in W(I, J, K)$, then $y \in \operatorname{Min}\left(w^{\prime}\right)$.

Proof. First, assume that $\ell(u s)=\ell(u)-1$. Let $u=s_{1} s_{2} \cdots s_{k}$ be a reduced expression of $u$ with $s_{k}=s$. Since $u^{\prime}<u$, there is a subword of $s_{1} s_{2} \cdots s_{k}$ which is a reduced expression for $u^{\prime}$, say $s_{i_{1}} s_{i_{2}} \cdots s_{i_{m}}, 1 \leq i_{1}<i_{2}<\cdots<i_{m} \leq k$. If $k=i_{m}$, then $u^{\prime} s s^{*}=s_{i_{1}} s_{i_{2}} \cdots s_{i_{m-1}} s^{*}$ which is a subexpression of the expression $u s s^{*}=s_{1} s_{2} \cdots s_{k-1} s^{*}$, the latter being reduced since $\ell\left(u s s^{*}\right)=\ell(u)$; hence $y:=$ $u^{\prime} s s^{*}$ satisfies $y<u s s^{*}$ and $\ell(y) \leq \ell\left(u^{\prime}\right)$. If $k \neq i_{m}$, then $u^{\prime}=s_{i_{1}} s_{i_{2}} \cdots s_{i_{m}}$ is a subexpression of the reduced expression $u s s^{*}=s_{1} s_{2} \cdots s_{k-1} s^{*}$; hence $y:=u^{\prime}$ satisfies $y<u s s^{*}$.

Now if $\ell(u s)=\ell(u)+1$, then taking a reduced expression $u=s_{1} \cdots s_{k}$, the exchange lemma implies that $u s s^{*}=s_{1} \cdots \widehat{s_{j}} \cdots s_{k} s$ for some $j \in\{1, \ldots, k\}$ (since $\left.\ell\left(u s s^{*}\right)=\ell(u)\right)$, hence $u s^{*}=s_{1} \cdots \widehat{s_{j}} \cdots s_{k}$ (since $s$ and $s^{*}$ commute). We then have $\ell\left(u s^{*}\right)=\ell(u)-1$, and we can argue as above replacing $s$ by $s^{*}$.

Corollary 8.10. Let $w, w^{\prime} \in W(I, J, K)$. The following conditions are equivalent.

(i) $w^{\prime}<_{\mathcal{O}} w$;

(ii) There are $u \in \operatorname{Min}(w)$ and $u^{\prime} \in\left[w^{\prime}\right]$ such that $u^{\prime}<u$;

(iii) For all $u \in \operatorname{Min}(w)$, there is $u^{\prime} \in\left[w^{\prime}\right]$ such that $u^{\prime}<u$.

Proof. The implications (iii) $\Rightarrow$ (i) $\Rightarrow$ (ii) immediately follow from the definition of the order $\leq_{\mathcal{O}}$. For showing (ii) $\Rightarrow\left(\right.$ iii), assume $u_{0} \in \operatorname{Min}(w)$ and $u_{0}^{\prime} \in\left[w^{\prime}\right]$ such that $u_{0}^{\prime}<u_{0}$, and let $u \in \operatorname{Min}(w)$. By Lemma 8.8, the element $u$ can be reached from $u_{0}$ by applying a sequence of elements of $\Theta$ at the right of $u_{0}$, without increasing the length. Applying Lemma 8.9 inductively, we find $u^{\prime} \in\left[w^{\prime}\right]$ such that $u^{\prime}<u$.

Let

$$
\mathcal{M}=\bigsqcup_{w \in W(I, J, K)} \operatorname{Min}(w) .
$$

In the rest of Section 8.2 , we establish some properties of the set $\mathcal{M}$. These properties are used in Section 8.3 to study the cover relations of the order $\leq_{\mathcal{O}}$.

For $v, w \in W$, we write $v \leq_{R} w$ if $\ell(w)=\ell\left(w v^{-1}\right)+\ell(v)$, that is, if $w$ has a reduced expression ending with a reduced expression of $v$ (this defines the so-called right weak order on $W$ ).

Given a subset $L \subset S$, by the parabolic decomposition $W=W^{L} W_{L}$, every $w \in W$ can be written as $w=w^{L} w_{L}$ with unique $w^{L} \in W^{L}$ and $w_{L} \in W_{L}$. Note that $w_{L}$ is also characterized as being the unique element in $W_{L}$ which is maximal with respect to $\leq_{R}$ and such that $w_{L} \leq_{R} w$.

Let $T \subset W$ denote the set $\bigcup_{w \in W} w S w^{-1}$, i.e., the set of reflections in $W$. For $u, u^{\prime} \in W$, we write $u^{\prime} \leq u$ if $u$ covers $u^{\prime}$ in the (strong) Bruhat order on $W$. Note that, in such a case, we must have $u^{\prime-1} u \in T$. 
Lemma 8.11. Let $L \subset S$. Let $w \in W, t \in T$ be such that $t \notin W_{L}$ and $w \leq w t$. Then $(w t)_{L} \leq_{R} w_{L}$.

Proof. We choose a reduced expression $u_{1} u_{2} \cdots u_{\ell} s_{1} s_{2} \cdots s_{k}$ of $w t$ such that $u_{1} \cdots u_{\ell}$ is a reduced expression of $(w t)^{L}$ and $s_{1} \cdots s_{k}$ is a reduced expression of $(w t)_{L}$. Since $w \lessdot w t$, we have that $w$ has a reduced expression which is obtained from $u_{1} u_{2} \cdots u_{\ell} s_{1} s_{2} \cdots s_{k}$ by deleting one letter. If the letter which is deleted is $s_{i}$ for some $i$, then $t=s_{k} s_{k-1} \cdots s_{i} s_{i+1} \cdots s_{k}$, which lies in $W_{L}$ (because the letters in a reduced expression of an element in a standard parabolic subgroup stay in this subgroup), a contradiction. Hence the letter which is deleted is among the $u_{i}$ 's. The element $w$ has therefore a reduced expression of the form

$$
u_{1} \cdots \widehat{u_{i}} \cdots u_{\ell} s_{1} s_{2} \cdots s_{k},
$$

hence it has $s_{1} s_{2} \cdots s_{k}=(w t)_{L}$ as a suffix, implying that $(w t)_{L} \leq_{R} w$. By maximality of $w_{L}$ with respect to $\leq_{R}$, we deduce that $(w t)_{L} \leq_{R} w_{L}$.

From now on, let $L=I \cup J \cup K$.

Lemma 8.12. We have

(a) $\mathcal{M}=\bigsqcup_{w \in W^{L}} w \cdot\left(\mathcal{M} \cap W_{L}\right)$. Hence $w \in \mathcal{M} \Leftrightarrow w_{L} \in \mathcal{M}$.

(b) $\mathcal{M} \cap W_{L}=\left\{u v^{*}: u, v \in W_{I}, \ell\left(u v^{-1}\right)=\ell(u)+\ell(v)\right\}$.

(c) If $W_{I}$ is finite, then

$$
\mathcal{M} \cap W_{L}=\left\{u v^{*}: u, v \in W_{I}, u \leq_{R} w_{0, I} v\right\},
$$

where $w_{0, I}$ denotes the longest element in $W_{I}$.

Proof. Part (a) immediately follows from Lemma 8.6(b).

By Lemma 8.6(a), the elements of $\mathcal{M} \cap W_{L}$ are exactly of the form $w x x^{*}$ with $w \in W_{I}=W(I, J, K) \cap W_{L}$ and $x \in W_{I}$ such that $\ell(w x)+\ell\left(x^{-1}\right)=\ell(w)$. Setting $u=w x$ and $v=x$, we get the description given in Part (b).

When $W_{I}$ is finite, the latter condition on lengths is equivalent to $\ell\left(w_{0, I} w^{-1}\right)+$ $\ell(w x)=\ell\left(w_{0, I} x\right)$, or to the fact that $w x \leq_{R} w_{0, I} x$. Setting $u=w x$ and $v=x$, we get the asserted condition $u \leq_{R} w_{0, I} v$.

We also have the following results.

Lemma 8.13. Let $L=I \cup J \cup K$ be as above. If $w \in \mathcal{M}$ and $y \in W$ are such that $y_{L} \leq_{R} w_{L}$, then $y \in \mathcal{M}$.

Proof. By Lemma 8.12, we have $w_{L}=u v^{*}$ with $u, v \in W_{I}$ such that $\ell\left(u v^{-1}\right)=$ $\ell(u)+\ell(v)$. If $y_{L} \leq_{R} w_{L}$, then since $I$ and $J$ are disconnected, we have $y_{L}=u^{\prime} v^{\prime *}$ with $u^{\prime} \leq_{R} u, v^{\prime} \leq_{R} v$. Setting $u=u_{1} u^{\prime}, v=v_{1} v^{\prime}$, we have

$$
\begin{aligned}
\ell\left(w_{L}\right)=\ell(u)+\ell(v) & =\ell\left(u^{\prime}\right)+\ell\left(u_{1}\right)+\ell\left(v^{\prime}\right)+\ell\left(v_{1}\right) \\
& \geq \ell\left(u_{1}\right)+\ell\left(u^{\prime} v^{\prime-1}\right)+\ell\left(v_{1}\right) \\
& \geq \ell\left(u_{1} u^{\prime} v^{\prime-1} v_{1}^{-1}\right)=\ell\left(u v^{-1}\right)=\ell(u)+\ell(v) .
\end{aligned}
$$

We deduce that $\ell\left(u^{\prime} v^{\prime-1}\right)=\ell\left(u^{\prime}\right)+\ell\left(v^{\prime}\right)$, which shows that $y_{L} \in \mathcal{M}$ (by Lemma 8.12(b)). Hence $y=y^{L} y_{L} \in \mathcal{M}$ by Lemma 8.12 (a).

Corollary 8.14. Let $w \in \mathcal{M}$. Let $t \in T \backslash W_{L}$ be such that $w \leq w t$. Then $w t \in \mathcal{M}$.

Proof. By Lemma 8.11 we have $(w t)_{L} \leq_{R} w_{L}$. By Lemma 8.13 we get $w t \in \mathcal{M}$. 
Lemma 8.15. Let $y \in \mathcal{M}, w \in W(I, J, K)$ be such that $y \leq w$ and $\ell(y)+2 \leq \ell(w)$. Then there is $v \in \mathcal{M}$ with $y<v<w$.

Proof. By [De77, Corollary 3.8], the poset $\left(W^{K}, \leq\right)$ is graded and the rank function is given by the restriction of $\ell$ to $W^{K}$. Since $\mathcal{M} \subset W^{K}$, there is $t \in T$ such that $y<w t \leq w$ and $w t \in W^{K}$.

Since $w \in W(I, J, K)$, we can write $w=w^{L} w_{L}$ with $w^{L} \in W^{L}$ and $w_{L} \in W_{I}$, and $\ell(w)=\ell\left(w^{L}\right)+\ell\left(w_{L}\right)$; hence a reduced expression of $w$ can be obtained by concatenating reduced expressions of $w^{L}$ and $w_{L}$. Since $w t \lessdot w$, we can get a reduced expression of $w t$ by deleting either a letter in $w^{L}$ or a letter in $w_{L}$. In the latter case, we have $w t \in W^{L} W_{I}=W(I, J, K)$, hence $w t \in \mathcal{M}$, and the lemma is obtained with $v=w t$. It remains to consider the former situation. In such a case, we have $w t=\left(w^{L} t_{0}\right) w_{L}$, where $w^{L} t_{0} \lessdot w^{L}$. Since $w^{L} \in W^{L}$, we must have $t_{0} \in T \backslash W_{L}$, and therefore

$$
t=w_{L}^{-1} t_{0} w_{L} \notin W_{L} .
$$

Let $y=y_{1} y_{2} y_{3}^{*}$ be the unique decomposition of $y$ with $y_{1} \in W^{L}, y_{2}, y_{3} \in W_{I}$; this follows from Lemma 8.12 (since $y \in \mathcal{M}$ ) or more generally from the fact that $y \in W^{K}$. Since $w t \in W^{K}$, we can decompose $w t=w_{1} w_{2} w_{3}^{*}$ in the same way. Since $y \leq w t$, there are $\hat{w}_{1}, \hat{w}_{2}, \hat{w}_{3}$ such that $y=\hat{w}_{1} \hat{w}_{2} \hat{w}_{3}^{*}, \hat{w}_{i} \leq w_{i}$ and $\ell(y)=\ell\left(\hat{w}_{1}\right)+\ell\left(\hat{w}_{2}\right)+\ell\left(\hat{w}_{3}\right)$ : just take a reduced expression of $w t$ which is obtained by concatenating reduced expressions of $w_{1}, w_{2}$ and $w_{3}^{*}$; it then has a subword which is a reduced expression of $y$. Note that since $\hat{w}_{2} \hat{w}_{3}^{*} \in W_{I \cup J} \subset W_{L}$, we have that $\ell\left(w_{1} \hat{w}_{2} \hat{w}_{3}^{*}\right)=\ell\left(w_{1}\right)+\ell\left(\hat{w}_{2}\right)+\ell\left(\hat{w}_{3}\right)$. Hence $y \leq w_{1} \hat{w}_{2} \hat{w}_{3}^{*} \leq w t$.

Claim 1. $w_{1} \hat{w}_{2} \hat{w}_{3}^{*} \in \mathcal{M}$.

To see this, note that since $\ell(y)=\ell\left(\hat{w}_{1}\right)+\ell\left(\hat{w}_{2} \hat{w}_{3}^{*}\right)$, we have $\hat{w}_{2} \hat{w}_{3}^{*} \leq_{R} y$, and so $\hat{w}_{2} \hat{w}_{3}^{*} \leq_{R} y_{L}=y_{2} y_{3}^{*}$ (since $\left.\hat{w}_{2} \hat{w}_{3}^{*} \in W_{L}\right)$. Thus $y_{2}=u_{2} \hat{w}_{2}, y_{3}=u_{3} \hat{w}_{3}$ for some $u_{2}, u_{3} \in W_{I}$ such that $\ell\left(y_{i}\right)=\ell\left(u_{i}\right)+\ell\left(\hat{w}_{i}\right)$ (using that $I$ and $J$ are disconnected). But since $y \in \mathcal{M}$, by Lemma 8.12 we have $\ell\left(y_{2} y_{3}^{-1}\right)=\ell\left(y_{2}\right)+\ell\left(y_{3}\right)$ and we get

$$
\begin{aligned}
\ell\left(u_{2}\right)+\ell\left(\hat{w}_{2} \hat{w}_{3}^{-1}\right)+\ell\left(u_{3}\right) & \geq \ell\left(u_{2} \hat{w}_{2} \hat{w}_{3}^{-1} u_{3}^{-1}\right)=\ell\left(y_{2} y_{3}^{-1}\right)=\ell\left(y_{2}\right)+\ell\left(y_{3}\right) \\
& =\ell\left(u_{2}\right)+\ell\left(\hat{w}_{2}\right)+\ell\left(\hat{w}_{3}\right)+\ell\left(u_{3}\right) \\
& \geq \ell\left(u_{2}\right)+\ell\left(\hat{w}_{2} \hat{w}_{3}^{-1}\right)+\ell\left(u_{3}\right),
\end{aligned}
$$

hence $\ell\left(\hat{w}_{2} \hat{w}_{3}^{-1}\right)=\ell\left(\hat{w}_{2}\right)+\ell\left(\hat{w}_{3}\right)$, implying Claim 1 (see Lemma 8.12).

Since $y \leq w_{1} \hat{w}_{2} \hat{w}_{3}^{*}<w$, if $y \neq w_{1} \hat{w}_{2} \hat{w}_{3}^{*}$, then we get the conclusion of the lemma with $v=w_{1} \hat{w}_{2} \hat{w}_{3}^{*}$. Hence we can assume that $y=w_{1} \hat{w}_{2} \hat{w}_{3}^{*}$.

Claim 2. We can find $t^{\prime} \in T, t^{\prime} \notin W_{L}$, such that $y \leq y t^{\prime}<w$.

Since $y$ has a reduced expression obtained from $w_{1} w_{2} w_{3}^{*}=w t$ by only deleting letters in $w_{2}$ and $w_{3}^{*}$, it follows that $w t=y t_{1} t_{2} \cdots t_{i}$ with $t_{j} \in W_{I \cup J} \cap T \subset W_{L}$ for all $j=1, \ldots, i$ and $y t_{1} t_{2} \cdots t_{j} \leq y t_{1} t_{2} \cdots t_{j+1}$ for all $j=1, \ldots, i-1$. Hence we have

$$
y t_{1} t_{2} \cdots t_{i-1} \lessdot y t_{1} t_{2} \cdots t_{i}=w t \lessdot w .
$$

Setting $u=y t_{1} t_{2} \cdots t_{i-1}$, we have $u \lessdot u t_{i}=w t \leq w=u t_{i} t$. By a property of Bruhat intervals (see [Dy91, Proposition 2.1 and its proof]), the Bruhat interval $[u, w]$ is isomorphic (as a poset) to a Bruhat interval in a dihedral reflection subgroup of $W$. Hence there is exactly one element $u^{\prime} \in W$ such that $u \leq u^{\prime} \leq w, u^{\prime} \neq w t$. Let $t_{i}^{\prime}, q \in T$ be such that $u^{\prime}=u t_{i}^{\prime}$ and $u^{\prime} q=w$. 
Subclaim. $t_{i}^{\prime} \notin W_{L}$.

We have $t_{i}^{\prime} q=t_{i} t \neq 1$ and, by [Dy91, Lemma 3.1], the reflection subgroup $W^{\prime}:=\left\langle t_{i}^{\prime}, q, t_{i}, t\right\rangle$ is dihedral. To show the Subclaim, arguing by contradiction, assume that $t_{i}^{\prime} \in W_{L}$. We show that this implies that $W^{\prime} \subset W_{L}$, contradicting (6). Note that if $W$ is of type $A$, this is clear as $W^{\prime}$ has to be either of type $A_{1} \times A_{1}$ or of type $A_{2}$, and is therefore generated by any two distinct reflections, whence $W^{\prime}=$ $\left\langle t_{i}, t_{i}^{\prime}\right\rangle \subset W_{L}$. In general the result can be proven as follows: by Dy91, Remark 3.2], the dihedral reflection subgroup $\left\langle t_{i}^{\prime}, t_{i}\right\rangle$ is included in a unique maximal dihedral reflection subgroup $W^{\prime \prime}$, defined by

$$
W^{\prime \prime}=\left\langle r_{\alpha} \mid \alpha \in\left(\mathbb{R} \alpha_{t_{i}^{\prime}}+\mathbb{R} \alpha_{t_{i}}\right) \cap \Phi^{+}\right\rangle,
$$

where $\Phi$ denotes a generalized root system for $(W, S)$ and $\alpha_{t_{i}}, \alpha_{t_{i}^{\prime}}$ are the roots attached to $t_{i}$ and $t_{i}^{\prime}$. Since $t_{i}^{\prime}, t_{i} \in W_{L}$ which is standard parabolic, it follows that for every root $\alpha \in\left(\mathbb{R} \alpha_{t_{i}^{\prime}}+\mathbb{R} \alpha_{t_{i}}\right) \cap \Phi^{+}$we have $r_{\alpha} \in W_{L}$, hence that $W^{\prime \prime} \subset W_{L}$. Since $W^{\prime \prime}$ is the unique maximal dihedral reflection subgroup containing both $t_{i}^{\prime}$ and $t_{i}$, it follows that $W^{\prime} \subset W^{\prime \prime} \subset W_{L}$. The proof of the Subclaim is then complete.

Arguing the same with $y t_{1} t_{2} \cdots t_{i-2} \lessdot y t_{1} t_{2} \cdots t_{i-2} t_{i-1} \leq y t_{1} t_{2} \cdots t_{i-2} t_{i-1} t_{i}^{\prime}=$ $u^{\prime}$, we find $t_{i-1}^{\prime} \notin W_{L}$ with $y t_{1} t_{2} \cdots t_{i-2} \lessdot y t_{1} t_{2} \cdots t_{i-2} t_{i-1}^{\prime} \sum_{y} t_{1} t_{2} \cdots t_{i-2} t_{i-1} t_{i}^{\prime}=$ $u^{\prime}$. Going on, we find reflections $t_{1}^{\prime}, t_{2}^{\prime}, \cdots, t_{i}^{\prime} \notin W_{L}$ such that

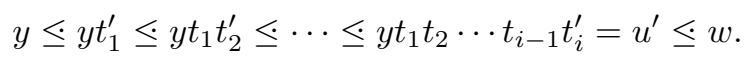

Hence, taking $t^{\prime}=t_{1}^{\prime}$, we get Claim 2 ,

Now it suffices to show that $y t^{\prime} \in \mathcal{M}$. To see this, as $t^{\prime} \notin W_{L}$, by Lemma 8.11, we get that $\left(y t^{\prime}\right)_{L} \leq_{R} y_{L}$. By Lemma 8.13, this implies that $y t^{\prime} \in \mathcal{M}$, as required.

8.3. Cover relations for $\leq_{\mathcal{O}}$. Given $w, w^{\prime} \in W(I, J, K)$, we write $w^{\prime} \varsigma_{\mathcal{O}} w$ if $w$ covers $w^{\prime}$ in the partial order $\leq_{\mathcal{O}}$. For $u, u^{\prime} \in W$, recall that we write $u^{\prime} \leq u$ if $u$ covers $u^{\prime}$ in the (strong) Bruhat order on $W$. We now characterize the cover relations in $\leq_{\mathcal{O}}$ in terms of elements of minimal length in cosets:

Theorem 8.16. Let $w, w^{\prime} \in W(I, J, K)$. The following are equivalent:

(i) We have $w^{\prime} \varsigma_{\mathcal{O}} w$;

(ii) There are $u \in \operatorname{Min}(w)$ and $u^{\prime} \in \operatorname{Min}\left(w^{\prime}\right)$ such that $u^{\prime} \leq u$;

(iii) There is $u^{\prime} \in \operatorname{Min}\left(w^{\prime}\right)$ such that $u^{\prime} \lessdot w$;

(iv) For all $u \in \operatorname{Min}(w)$, there is $u^{\prime} \in \operatorname{Min}\left(w^{\prime}\right)$ such that $u^{\prime} \lessdot u$.

Proof. It is clear that (iv) $\Rightarrow$ (iii) $\Rightarrow$ (ii). The fact that (ii) $\Rightarrow$ (i) follows from Corollary 8.10, noting that $\ell\left(w^{\prime}\right)=\ell\left(u^{\prime}\right)=\ell(u)-1=\ell(w)-1$, which forces $w^{\prime} \varsigma_{\mathcal{O}} w$. A similar argument shows (iii) $\Rightarrow$ (iv).

It remains to show that (i) $\Rightarrow$ (iii). If $w^{\prime} \varsigma_{\mathcal{O}} w$, then by Lemma 8.7 there is $y \in \operatorname{Min}\left(w^{\prime}\right)$ such that $y \leq w$. We claim that $w$ covers $y$ in Bruhat order, so that (iii) holds. Otherwise, we have $\ell(y)+2 \leq \ell(w)$, and Lemma 8.15yields $v \in \mathcal{M}$, say $v \in \operatorname{Min}\left(w^{\prime \prime}\right)$, such that $y<v<w$. Then by definition of $\leq_{\mathcal{O}}$ we have $w^{\prime \prime}<_{\mathcal{O}} w$, and by Corollary 8.10 we get $w^{\prime}<_{\mathcal{O}} w^{\prime \prime}$ : this contradicts $w^{\prime} \check{\complement}_{\mathcal{O}} w$.

Corollary 8.17. The poset $\left(W(I, J, K), \leq_{\mathcal{O}}\right)$ is graded by the restriction of the length function to $W(I, J, K)$.

Proof. In view of Theorem 8.16, we have that if $w, w^{\prime} \in W(I, J, K)$ satisfy $w^{\prime} \varsigma_{\mathcal{O}} w$, then $\ell\left(w^{\prime}\right)=\ell(w)-1$. This concludes the proof. 


\section{InCLUSions of ORBit CLOSURES In type $A$}

The purpose of this section is to illustrate the combinatorial results obtained in Section 8 in a situation that is related to the framework considered in Parts 112.

In this section, let $e \in \mathfrak{g l}_{n}(\mathbb{K})$ be a 2-nilpotent matrix of rank $r \leq \frac{n}{2}$, say

$$
e=\left(\begin{array}{ccc}
0 & 0 & 1_{r} \\
0 & 0 & 0 \\
0 & 0 & 0
\end{array}\right)
$$

so that the stabilizer $Z:=Z_{G}(e)$, with $G=\mathrm{GL}_{n}(\mathbb{K})$, is given by

$$
Z=\left\{\left(\begin{array}{ccc}
a & * & * \\
0 & b & * \\
0 & 0 & a
\end{array}\right): a \in \mathrm{GL}_{r}(\mathbb{K}), b \in \mathrm{GL}_{n-2 r}(\mathbb{K})\right\} .
$$

In the notation of Section 8, let $W(I, J, K) \subset \mathfrak{S}_{n}$, with $I=\left\{s_{1}, \ldots, s_{r-1}\right\}, J=$ $\left\{s_{n-r+1}, \ldots, s_{n-1}\right\}, K=\left\{s_{r+1}, \ldots, s_{n-r-1}\right\}$. The isomorphism $W_{I} \rightarrow W_{J}, x \mapsto x^{*}$ is given by $s_{i}^{*}=s_{n-r+i}$. Hence

$$
\begin{aligned}
W(I, J, K) & =W^{J \cup K} \\
& =\left\{w \in \mathfrak{S}_{n}: w(r+1)<\ldots<w(n-r), w(n-r+1)<\ldots<w(n)\right\} .
\end{aligned}
$$

We also consider the partial order $\leq_{\mathcal{O}}$ on $W(I, J, K)$ (see Proposition 8.1). Let $B \subset \mathrm{GL}_{n}(\mathbb{K})$ be the Borel subgroup of invertible upper-triangular matrices.

Theorem 9.1. With the above notation, there is a one-to-one correspondence $w \mapsto$ $\mathbb{O}_{w}$ (resp. $w \mapsto \mathcal{O}_{w}$ ) between the set $W(I, J, K)$ and the set of $Z$-orbits on $G / B$ (resp. the set of B-orbits on $G \cdot e)$. Moreover,

$$
\mathbb{O}_{w^{\prime}} \subset \overline{\mathbb{O}_{w}} \quad\left(\text { resp. } \mathcal{O}_{w^{\prime}} \subset \overline{\mathcal{O}_{w}}\right) \quad \Leftrightarrow \quad w^{\prime} \leq_{\mathcal{O}} w .
$$

In particular, the cover relations for the inclusions of orbit closures are described in Theorem 8.16 .

The orbit $G \cdot e \subset \mathfrak{g l}_{n}(\mathbb{K})$ is the set of 2-nilpotent matrices of rank $r$. The topology of the $B$-orbits on the set of 2-nilpotent matrices has been studied in BP19, BR12. In particular the parametrization of orbits and the characterization of the inclusion relations between orbit closures given in Theorem 9.1 is essentially given in BP19, Lemma 7.3.1]; however, we have not understood all the arguments given in BP19]: see the comment after Lemma 9.4. For the sake of completeness, we give a proof of Theorem 9.1 which is mainly based on BR12. We prove Theorem 9.1 in two steps: in Section 9.1, we first define the bijective map $w \mapsto \mathcal{O}_{w}$. In Section 9.2, we prove the assertion on inclusion of orbit closures. To do this, we first recall Boos-Reineke's criterion for inclusion of orbit closures, and we then show the equivalence between this criterion and the inequality $w^{\prime} \leq_{\mathcal{O}} w$ (Proposition 9.9), by using the version of Boos-Reineke's criterion stated in Lemma 9.7. We only prove the result claimed for the $B$-orbits on $G \cdot e$. The analogous claim for the $Z$-orbits on $G / B$ ensues, due to the correspondence between these two orbit sets; see also Remark 9.3 .

We point out that the characterization of the cover relations obtained in Theorem 9.1 appears to be new. In BR12, Theorem 4.6], the authors give a list of elementary relations which include all the cover relations, but the obtained characterization is only necessary and not sufficient. 
9.1. Parametrization of orbits by oriented link patterns. In [BR12], the $B$ orbits on 2-nilpotent matrices in $\mathfrak{g l}_{n}(\mathbb{K})$ are parametrized by oriented link patterns. Let $\mathcal{D}_{n}$ be the set of oriented link patterns on $\{1,2, \ldots, n\}$, that is, oriented graphs on the set $\{1,2, \ldots, n\}$ such that every vertex is incident with at most one arrow. Let $\mathcal{D}_{n, r} \subset \mathcal{D}_{n}$ denote the subset of oriented link patterns with $r$ arrows.

Hereafter, $\left\{\varepsilon_{1}, \ldots, \varepsilon_{n}\right\}$ denotes the standard basis of $\mathbb{K}^{n}$. Given $d \in \mathcal{D}_{n}$, a representative of the corresponding $B$-orbit $\mathcal{O}_{d}$ is given by the matrix $M_{d} \in \mathfrak{g l}_{n}(\mathbb{K})$ defined by $M_{d}\left(\varepsilon_{i}\right)=\varepsilon_{j}$ if there is an arrow from $i$ to $j$ and $M_{d}\left(\varepsilon_{i}\right)=0$ if there is no arrow starting from $i$.

For $k \in\{1, \ldots, n\}$, we write $p_{k}^{d}$ for the number of vertices to the left of $k$ (i.e., $\leq k$ ) which are not incident with an arrow, plus the number of arrows whose target vertex lies to the left of $k$. For $k \in\{0,1, \ldots, n\}$ and $\ell \in\{1, \ldots, n\}$, we write $q_{k, \ell}^{d}$ for $p_{\ell}^{d}$ plus the number of arrows whose source vertex lies to the left of $\ell$ and target vertex lies to the left of $k$. Note that $q_{0, \ell}^{d}=p_{\ell}^{d}$. Then Boos and Reineke show:

Theorem 9.2 ([BR12, Theorem 4.3]). The set of 2-nilpotent matrices is the disjoint union of the orbits $\mathcal{O}_{d}$ for $d \in \mathcal{D}_{n}$. Let $d, d^{\prime} \in \mathcal{D}_{n}$, and let us write $d^{\prime} \leq_{\mathcal{D}} d$ if $\overline{\mathcal{O}_{d^{\prime}}} \subset \overline{\mathcal{O}_{d}}$. Then we have $d^{\prime} \leq_{\mathcal{D}} d$ if and only if $q_{k, \ell}^{d} \leq q_{k, \ell}^{d^{\prime}}$ for all $k \in\{0,1, \ldots, n\}$ and all $\ell \in\{1, \ldots, n\}$.

For $e$ as in (7), the orbit $G \cdot e$ is the set of 2-nilpotent matrices of rank $r$; hence we get

$$
G \cdot e=\bigsqcup_{d \in \mathcal{D}_{n, r}} \mathcal{O}_{d}
$$

Then, to obtain the parametrization of the $B$-orbits on $G \cdot e$ claimed in Theorem 9.1, we construct an explicit bijection between the sets $W(I, J, K)$ and $\mathcal{D}_{n, r}$.

The matrix $e$ of (7) is of the form $M_{d_{1}}$, where $d_{1}$ is the oriented link pattern with an arrow from $n-r+i$ to $i$ for all $i \in\{1, \ldots, r\}$. For every $w \in W=\mathfrak{S}_{n}$, identifying $w$ with its permutation matrix $w \in \mathrm{GL}_{n}(\mathbb{K})$, the matrix $w \cdot e$ is also of the form $M_{d_{w}}$ for a unique oriented link pattern $d_{w} \in \mathcal{D}_{n, r}$. Specifically, $d_{w}$ has an arrow from $w(n-r+i)$ to $w(i)$ for all $i \in\{1, \ldots, r\}$; in other words, $d_{w}$ is the oriented link pattern obtained from $d_{1}$ by letting $w$ act on the set of vertices $\{1, \ldots, n\}$ in the canonical way.

Moreover, if $d_{w}=d_{w^{\prime}}$, then $w \cdot e=w^{\prime} \cdot e$; hence $w^{-1} w$ centralizes $e$. In view of (8) , the latter fact implies that the permutation $w^{\prime-1} w$ is of the form $y x x^{*}$ with $y \in W_{K}$ and $x \in W_{I}$, that is, $w^{\prime-1} w$ belongs to the subgroup $W_{K} W_{I, J} \subset \mathfrak{S}_{n}$ with the notation of Section 8 . Since $W(I, J, K)$ contains exactly one representative for each $W_{K} W_{I, J}$-coset, this yields an injective map

$$
W(I, J, K) \rightarrow \mathcal{D}_{n, r}, w \mapsto d_{w} .
$$

Finally, since one has $\left|\mathcal{D}_{n, r}\right|=\frac{n !}{r !(n-2 r) !}=\left|W^{J \cup K}\right|=|W(I, J, K)|$, the above map is bijective. This yields the bijection $w \mapsto \mathcal{O}_{w}:=B w \cdot e=\mathcal{O}_{d_{w}}$ of Theorem 9.1

Remark 9.3. The set of $B$-orbits on $G \cdot e \cong G / Z$ and the set of $Z$-orbits on $\mathcal{B}=G / B$ are in bijection via the map $B g \cdot e \mapsto Z g^{-1} \cdot B$, and this bijection relates the parametrization of the former set of orbits given above with the parametrization of the latter set of orbits given in Example 7.3. Specifically, every element $w \in$ $W(I, J, K)$ can be written as $w=w_{1} w_{2}$ for a unique pair $\left(w_{1}, w_{2}\right) \in W^{I \cup J \cup K} \times W_{I}$, and the $B$-orbit $\mathcal{O}_{w}=B w \cdot e$ corresponds to the $Z$-orbit $Z w^{-1} \cdot B$ attached to the pair $\left(w_{1}^{-1}, w_{2}^{-1}\right) \in{ }^{P} W \times \mathfrak{S}_{r}$, with the notation of Example 7.3 
9.2. Inclusion relations between orbit closures. Let $0=V_{0} \subset V_{1} \subset \ldots \subset$ $V_{n}=\mathbb{K}^{n}$ be the standard complete flag of $\mathbb{K}^{n}$, so that $B=\left\{g \in G: g\left(V_{i}\right)=V_{i}, \forall i\right\}$. Given a 2-nilpotent matrix $y \in \mathfrak{g l}_{n}(\mathbb{K})$ and $(i, j) \in\{1, \ldots, n\} \times\{0,1, \ldots, n\}$, we write

$$
r(i, j, y):=\operatorname{dim}\left(y\left(V_{i}\right)+V_{j}\right)
$$

Note that the mapping $y \mapsto r(i, j, y)$ is constant on every $B$-orbit.

Lemma 9.4. Let $d, d^{\prime} \in \mathcal{D}_{n, r}$ and write $w, w^{\prime}$ for the corresponding elements in $W(I, J, K)$ in the sense of Section 9.1 , i.e., $d=d_{w}$ and $d^{\prime}=d_{w^{\prime}}$. We have $d^{\prime} \leq_{\mathcal{D}} d$ (that is, $\overline{\mathcal{O}_{w^{\prime}}} \subset \overline{\mathcal{O}_{w}}$; see the notation in Theorem 9.2 ) if and only if $r\left(i, j, w^{\prime} \cdot e\right) \leq r(i, j, w \cdot e)$ for all $i, j$. In particular, the orbit closure $\overline{\mathcal{O}_{w}}$, where $w \in W(I, J, K)$, is given by

$$
\overline{\mathcal{O}_{w}}=\{y \in G \cdot e: r(i, j, y) \leq r(i, j, w \cdot e) \quad \forall(i, j) \in\{1, \ldots, n\} \times\{0,1, \ldots, n\}\} .
$$

The description of $\overline{\mathcal{O}_{w}}$ given in Lemma 9.4 already appears in BP19, Lemma 7.3.5]. However, while we understand the description of the $B$-orbit given in the proof of [BP19, Lemma 7.3.5], we do not see how to deduce the given description of the closure. This proof also contains a reference to Rothbach's thesis Ro09, but for the sake of completeness we prove Lemma 9.4 by using Theorem 9.2

Proof. Let $y=w \cdot e$ and $y^{\prime}=w^{\prime} \cdot e$. Assume that $d^{\prime} \leq_{\mathcal{D}} d$. By Theorem 9.2 we have $q_{k, \ell}^{d} \leq q_{k, \ell}^{d^{\prime}}$ for all $k, \ell$. But $q_{k, \ell}^{d}$ is $\operatorname{simply} \operatorname{dim}\left(V_{\ell} \cap \operatorname{ker}(y)\right)+\operatorname{dim}\left(y\left(V_{\ell}\right) \cap V_{k}\right)$; hence the inequality gives

$$
\begin{aligned}
& \operatorname{dim}\left(V_{\ell} \cap \operatorname{ker}(y)\right)+\operatorname{dim} y\left(V_{\ell}\right)-\operatorname{dim}\left(V_{k}+y\left(V_{\ell}\right)\right) \\
\leq \quad & \operatorname{dim}\left(V_{\ell} \cap \operatorname{ker}\left(y^{\prime}\right)\right)+\operatorname{dim} y^{\prime}\left(V_{\ell}\right)-\operatorname{dim}\left(V_{k}+y^{\prime}\left(V_{\ell}\right)\right) .
\end{aligned}
$$

But $\operatorname{dim} y\left(V_{\ell}\right)+\operatorname{dim}\left(V_{\ell} \cap \operatorname{ker}(y)\right)=\ell$; hence the inequality can be rewritten as

$$
\operatorname{dim}\left(V_{k}+y^{\prime}\left(V_{\ell}\right)\right) \leq \operatorname{dim}\left(V_{k}+y\left(V_{\ell}\right)\right),
$$

which implies that $r\left(\ell, k, y^{\prime}\right) \leq r(\ell, k, y)$ for all $k \in\{0, \ldots, n\}, \ell \in\{1, \ldots, n\}$.

Conversely, assuming that $r\left(\ell, k, y^{\prime}\right) \leq r(\ell, k, y)$ for all $k \in\{0, \ldots, n\}, \ell \in$ $\{1, \ldots, n\}$, we can establish $q_{k, \ell}^{d} \leq q_{k, \ell}^{d^{\prime}}$ by the same inequalities as above, going the other way around.

Hence we have $d^{\prime} \leq_{\mathcal{D}} d$ if and only if $r\left(\ell, k, y^{\prime}\right) \leq r(\ell, k, y)$ for all $k \in\{0, \ldots, n\}$, $\ell \in\{1, \ldots, n\}$. The claimed description of orbit closures ensues.

We now give an alternative combinatorial criterion to determine whether an orbit is included in the closure of another orbit.

Notation 9.5. Let $e$ be as in (7). Let $w \in \mathfrak{S}_{n}$ and let $e_{w}=w \cdot e=w e w^{-1}$. Associate a sequence $S_{w}$ of integers to $w \in \mathfrak{S}_{n}$ as follows: the $i$-th number in the sequence is $j$ if $e_{w}\left(\varepsilon_{i}\right)=\varepsilon_{j}$ and 0 if $e_{w}\left(\varepsilon_{i}\right)=0$. Hence, for all $i=n-r+1, \ldots, n$, this sequence has the number $w(i-(n-r))$ in position $w(i)$, and zero everywhere else. In particular the sequence has $r$ nonzero entries, all distinct.

Example 9.6. For $n=4$ and $r=2$, let $w=s_{2} s_{1} s_{3} s_{2}=(1,3)(2,4)$. Then $S_{w}=(3400)$.

Note that if $S_{w}$ has $k$ as nonzero entry, then $S_{w}$ has 0 as $k$-th entry. In the following statement, for every $i \in\{1, \ldots, n\}$, we denote by $S_{w}^{i}$ the truncated sequence formed by the nonzero entries which are within the first $i$ entries of $S_{w}$. 
Lemma 9.7. Let $w, w^{\prime} \in W(I, J, K)$, and let $d_{w}, d_{w^{\prime}}$ be the corresponding oriented link patterns. We have $d_{w^{\prime}} \leq_{\mathcal{D}} d_{w}$ if and only if

$$
\left|\left\{k \in S_{w^{\prime}}^{i}: k>j\right\}\right| \leq\left|\left\{k \in S_{w}^{i}: k>j\right\}\right| \text { for all } i \in\{1, \ldots, n\} \text {, all } j \geq 0 .
$$

Proof. This follows immediately from Lemma 9.4 and from the fact that

$$
\begin{aligned}
r(i, j, w \cdot e) & =\operatorname{dim} e_{w}\left(V_{i}\right)+\operatorname{dim} V_{j}-\operatorname{dim} e_{w}\left(V_{i}\right) \cap V_{j} \\
& =\left|S_{w}^{i}\right|+j-\left|\left\{k \in S_{w}^{i}: 1 \leq k \leq j\right\}\right| .
\end{aligned}
$$

Example 9.8. Let $n=4$ and $r=2$, so that $I=\left\{s_{1}\right\}, J=\left\{s_{3}\right\}, K=\emptyset$.

(a) Let $w=s_{2} s_{1} s_{3} s_{2}=(1,3)(2,4)$ be as in Example 9.6 and let $w^{\prime}=s_{2} s_{1}=$ $(1,3,2)$. Both elements lie in $W(I, J, K)$. We have $S_{w}=(3400)$ and $S_{w^{\prime}}=$ (0 3001 ), hence

$$
S_{w}^{1}=(3), S_{w}^{2}=(34)=S_{w}^{3}=S_{w}^{4} \quad \text { and } \quad S_{w^{\prime}}^{1}=\emptyset, S_{w^{\prime}}^{2}=(3)=S_{w^{\prime}}^{3}, S_{w^{\prime}}^{4}=(31) .
$$

Hence $d_{w^{\prime}} \leq_{\mathcal{D}} d_{w}$, that is, $\overline{\mathcal{O}_{w^{\prime}}} \subset \overline{\mathcal{O}_{w}}$.

(b) The sequences $S_{w}$ associated to the various elements $w \in W(I, J, K)$ and the corresponding inclusion relations between orbit closures are described in Figure 2.

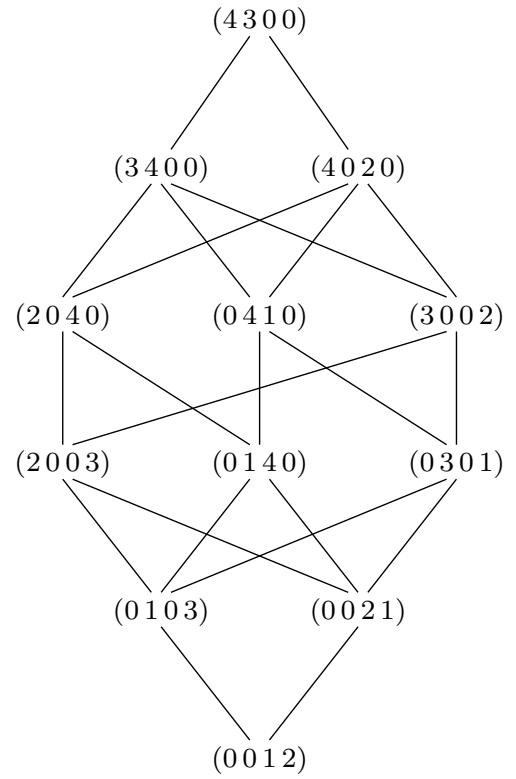

FIgURE 2. The sequences $S_{w}$ and the inclusion relations between orbit closures in type $A_{3}$ for $r=2$. Here $I=\left\{s_{1}\right\}, J=\left\{s_{3}\right\}$, $K=\emptyset$.

We aim to prove the claim on inclusion of orbit closures in Theorem 9.1 by using the criterion from Lemma 9.7. We will use extensively the tableau criterion for the strong Bruhat order on the symmetric group (see for instance [BB96]): if $x \in \mathfrak{S}_{n}$, we write $x=x_{1} x_{2} \cdots x_{n}$ if $x(i)=x_{i}$. This is called the line notation of $x$. Then given $x, y \in \mathfrak{S}_{n}$, we have $x \leq y$ (here $\leq$ denotes the strong Bruhat order) if and only if whenever $1 \leq k \leq i$, we have $x_{k, i} \leq y_{k, i}$, where $x_{k, i}$ is the $k$-th entry of the sequence obtained from $x_{1} x_{2} \cdots x_{i}$ by reordering the entries increasingly. 
Let $w, w^{\prime} \in W(I, J, K)$. We consider the partial order $\leq_{\mathcal{O}}$ of Proposition 8.1. Recall that, by definition, the relation $w^{\prime} \leq_{\mathcal{O}} w$ holds if there is $u \in\left[w^{\prime}\right]=w^{\prime} W_{K} W_{I, J}$ such that $u \leq w$. Note that this is not equivalent to having $w^{\prime} \leq w$ as there might be several elements of minimal length in the coset $\left[w^{\prime}\right]$ (see Section 8).

The proof of Theorem 9.1 is complete once we show:

Proposition 9.9. We have $w^{\prime} \leq_{\mathcal{O}} w$ if and only if $d_{w^{\prime}} \leq_{\mathcal{D}} d_{w}$.

Proof. Assume that $w^{\prime} \leq_{\mathcal{O}} w$. For showing that $d_{w^{\prime}} \leq_{\mathcal{D}} d_{w}$, fix $i \in\{1, \ldots, n\}$ and $j \geq 0$, and let us check the condition of Lemma 9.7 on the sequences $S_{w}^{i}$ and $S_{w^{\prime}}^{i}$.

Since $w=w_{1} w_{2} \cdots w_{n} \in W(I, J, K)=W^{J \cup K}$, we have that the last $r$ entries in the sequence are increasing. Note that they give the positions of the nonzero entries in $S_{w}$, and that these entries are $w_{1}, w_{2}, \ldots, w_{r}$. Since $w_{n-r+1}<w_{n-r+2}<$ $\cdots<w_{n}$, the entries $w_{k}$ with $k \geq n-r+1$ and $w_{k} \leq i$ form a subsequence of the form $w_{n-r+1} w_{n-r+2} \cdots w_{n-r+\ell}$ for some $\ell$. This implies that the sequence $S_{w}^{i}$ is

$$
S_{w}^{i}=\left(w_{1} w_{2} \cdots w_{\ell}\right) .
$$

The relation $w^{\prime} \leq_{\mathcal{O}} w$ means that there is $u \in\left[w^{\prime}\right]$ such that $u \leq w$. Since $u$ belongs to the coset $\left[w^{\prime}\right]=w^{\prime} W_{K} W_{I, J}$, there is $z \in \mathfrak{S}_{r}$ such that

$$
u(n-r+k)=w^{\prime}\left(n-r+z_{k}\right) \quad \text { and } \quad u(k)=w^{\prime}\left(z_{k}\right) \text { for all } k \in\{1, \ldots, r\},
$$

and this implies that $S_{u}=S_{w^{\prime}}$, hence $S_{u}^{i}=S_{w^{\prime}}^{i}$.

Since $u \leq w$, we must have $u_{m}>i$ whenever $m>n-r+\ell$; otherwise we would have a contradiction with the tableau criterion. (Indeed, the integers $w_{k, n-r+\ell}$ for $1 \leq k \leq n-r+\ell$ contain all the integers from 1 to $i$, whereas for the integers $u_{k, n-r+\ell}$, the integer $u_{m}$ would be missing.) It follows that the entries of the sequence $S_{u}^{i}$ are among $u_{1}, u_{2}, \ldots, u_{\ell}$. Since $u \leq w$, it follows from the tableau criterion that

$$
\left|\left\{k \in\left\{u_{1}, \ldots, u_{\ell}\right\}: k>j\right\}\right| \leq\left|\left\{k \in\left\{w_{1}, \ldots, w_{\ell}\right\}: k>j\right\}\right| .
$$

Since $S_{w}^{i}=\left(w_{1} w_{2} \cdots w_{\ell}\right)$ and $S_{u}^{i}$ has entries among $u_{1}, u_{2}, \ldots, u_{\ell}$, we get that

$$
\left|\left\{k \in S_{w^{\prime}}^{i}: k>j\right\}\right|=\left|\left\{k \in S_{u}^{i}: k>j\right\}\right| \leq\left|\left\{k \in S_{w}^{i}: k>j\right\}\right| .
$$

The proof of the first implication is complete.

To show the converse, we use BR12, Theorem 4.6], where the covering relations in the poset $\left(\mathcal{D}_{n}, \leq_{\mathcal{D}}\right)$ are described. We may and will assume that there is a covering relation between $d_{w}$ and $d_{w^{\prime}}$, and we have to prove that $w^{\prime} \leq_{\mathcal{O}} w$. According to [BR12, Theorem 4.6], we have three types of relations to consider.

For the first type, we assume that for some $1 \leq a<b \leq n$, there is an arrow $a \rightarrow b$ in $d_{w}$ whereas there is an arrow $b \rightarrow a$ in $d_{w^{\prime}}$. We also assume that the other arrows are the same for $d_{w}$ and for $d_{w^{\prime}}$. From the definition of $d_{w}$, it follows that there is an integer $i$ such that $1 \leq i \leq r, w_{n-r+i}=a$ and $w_{i}=b$. Moreover, setting $u=(a, b) \circ w$, we have $d_{u}=d_{w^{\prime}}$; hence $u$ belongs to the coset $\left[w^{\prime}\right]=w^{\prime} W_{K} W_{I, J}$ (see Section 9.1). Since $a<b$ and $w^{-1}(a)>w^{-1}(b)$, we get $u \leq w$, and so $w^{\prime} \leq_{\mathcal{O}} w$.

For the second type, we are given three integers $1 \leq a<b<c \leq n$ such that the only difference between $d_{w}$ and $d_{w^{\prime}}$ involves the vertices $a, b, c$ and corresponds to one edge of the second diagram in BR12, Theorem 4.6]. In each case, we note that $d_{w^{\prime}}$ can be obtained from $d_{w}$ by switching two vertices $a^{\prime}<b^{\prime}$ among $a, b, c$, hence $d_{w^{\prime}}=d_{\left(a^{\prime}, b^{\prime}\right) \circ w}$, which yields $u:=\left(a^{\prime}, b^{\prime}\right) \circ w \in\left[w^{\prime}\right]$. Moreover, in each case, it turns out that $w^{-1}\left(a^{\prime}\right)>w^{-1}\left(b^{\prime}\right)$ (where we use that $w^{-1}(i)$ lies in $\{1, \ldots, r\}$, 
$\{n-r+1, \ldots, n\}$, or $\{r+1, \ldots, n-r\}$ depending on whether $i$ is end point of an arrow, starting point of an arrow, or not incident with any arrow in $d_{w}$ ). This implies that $u \leq w$, and therefore $w^{\prime} \leq_{\mathcal{O}} w$.

For the third type, there are $1 \leq a<b<c<d \leq n$ such that the only difference between $d_{w}$ and $d_{w^{\prime}}$ involves the vertices $a, b, c, d$, and corresponds to one of the edges of the third diagram in [BR12, Theorem 4.6]. Specifically, two cases may arise:

(a) $d_{w^{\prime}}$ is obtained from $d_{w}$ by switching two vertices $a^{\prime}<b^{\prime}$ (among $a, b, c, d$ ) such that $a^{\prime}$ is the starting point of an arrow in $d_{w}$ and $b^{\prime}$ is the end point of an arrow in $d_{w}$. In that case, we get $d_{w^{\prime}}=d_{\left(a^{\prime}, b^{\prime}\right) \circ w}$; hence $u:=\left(a^{\prime}, b^{\prime}\right) \circ w$ belongs to the coset $\left[w^{\prime}\right]$, and we have $u \leq w$, because $w^{-1}\left(a^{\prime}\right)>n-r \geq r \geq w^{-1}\left(b^{\prime}\right)$.

(b) $d_{w}$ has two arrows $a^{\prime} \rightarrow a^{\prime \prime}$ and $b^{\prime} \rightarrow b^{\prime \prime}$ with $a^{\prime}<b^{\prime}, b^{\prime \prime}<a^{\prime \prime}$, and $d_{w^{\prime}}$ is obtained from $d_{w}$ by switching the two starting points $a^{\prime}, b^{\prime}$; or, equivalently, by switching the two end points $a^{\prime \prime}, b^{\prime \prime}$. Then, the elements $u^{\prime}:=\left(a^{\prime}, b^{\prime}\right) \circ w$ and $u^{\prime \prime}:=\left(a^{\prime \prime}, b^{\prime \prime}\right) \circ w$ both belong to the coset $\left[w^{\prime}\right]$. Moreover, it follows from the definition of $d_{w}$ that $w^{-1}\left(a^{\prime}\right)=n-r+w^{-1}\left(a^{\prime \prime}\right)$ and $w^{-1}\left(b^{\prime}\right)=n-r+w^{-1}\left(b^{\prime \prime}\right)$. Hence, we have either $w^{-1}\left(a^{\prime}\right)>w^{-1}\left(b^{\prime}\right)$ or $w^{-1}\left(b^{\prime \prime}\right)>w^{-1}\left(a^{\prime \prime}\right)$, and thereby $u^{\prime} \leq w$ or $u^{\prime \prime} \leq w$.

In each case, the coset $\left[w^{\prime}\right]$ contains an element $u$ such that $u \leq w$. Therefore, $w^{\prime} \leq_{\mathcal{O}} w$. Note also that the third diagram in [BR12, Theorem 4.6] is the same as the diagram of Figure 2 and that it also coincides with the diagram of Figure 1 where the covering relations within the corresponding poset $\left(W(I, J, K), \leq_{\mathcal{O}}\right)$ are given.

\section{ACKNOWLEDGMENT}

The authors would like to thank Jacopo Gandini for valuable comments which helped improving this paper.

\section{REFERENCES}

[BP19] M. Bender and N. Perrin, Singularities of closures of B-conjugacy classes of nilpotent elements of height 2, Transform. Groups 24 (2019), no. 3, 741-768, DOI 10.1007/s00031-018-9505-6. MR3989689

[Bi73] A. Białynicki-Birula, Some theorems on actions of algebraic groups, Ann. of Math. (2) 98 (1973), 480-497, DOI 10.2307/1970915. MR366940

[BB96] Anders Björner and Francesco Brenti, An improved tableau criterion for Bruhat order, Electron. J. Combin. 3 (1996), no. 1, Research Paper 22. MR1399399

[BCE19] Magdalena Boos, Giovanni Cerulli Irelli, and Francesco Esposito, Parabolic orbits of 2-nilpotent elements for classical groups, J. Lie Theory 29 (2019), no. 4, 969-996. $\operatorname{MR} 4000994$

[BR12] Magdalena Boos and Markus Reineke, B-orbits of 2-nilpotent matrices and generalizations, Highlights in Lie algebraic methods, Progr. Math., vol. 295, Birkhäuser/Springer, New York, 2012, pp. 147-166, DOI 10.1007/978-0-8176-8274-3_6. MR2866850

[BC10] Paolo Bravi and Stéphanie Cupit-Foutou, Classification of strict wonderful varieties (English, with English and French summaries), Ann. Inst. Fourier (Grenoble) 60 (2010), no. 2, 641-681. MR2667789

[Br99] M. Brion, Rational smoothness and fixed points of torus actions, Transform. Groups 4 (1999), no. 2-3, 127-156, DOI 10.1007/BF01237356. Dedicated to the memory of Claude Chevalley. MR:1712861

[Br01] Michel Brion, On orbit closures of spherical subgroups in flag varieties, Comment. Math. Helv. 76 (2001), no. 2, 263-299, DOI 10.1007/PL00000379. MR1839347 
[CMcG93] David H. Collingwood and William M. McGovern, Nilpotent orbits in semisimple Lie algebras, Van Nostrand Reinhold Mathematics Series, Van Nostrand Reinhold Co., New York, 1993. MR 1251060

[De77] Vinay V. Deodhar, Some characterizations of Bruhat ordering on a Coxeter group and determination of the relative Möbius function, Invent. Math. 39 (1977), no. 2, 187-198, DOI 10.1007/BF01390109. MR435249

[Dy91] Matthew Dyer, On the "Bruhat graph" of a Coxeter system, Compositio Math. 78 (1991), no. 2, 185-191. MR1104786

[GMP17] Jacopo Gandini, Pierluigi Möseneder Frajria, and Paolo Papi, Spherical nilpotent orbits and abelian subalgebras in isotropy representations, J. Lond. Math. Soc. (2) 95 (2017), no. 1, 323-352, DOI 10.1112/jlms.12022. MR3653095

[GMP20] Jacopo Gandini, Pierluigi Möseneder Frajria, and Paolo Papi, Nilpotent orbits of height 2 and involutions in the affine Weyl group, Indag. Math. (N.S.) 31 (2020), no. 4, 568594, DOI 10.1016/j.indag.2020.04.006. MR 4126755

[Hu75] James E. Humphreys, Linear algebraic groups, Graduate Texts in Mathematics, No. 21, Springer-Verlag, New York-Heidelberg, 1975. MR.0396773

[Kac83] Victor G. Kac, Infinite-dimensional Lie algebras: An introduction, Progress in Mathematics, vol. 44, Birkhäuser Boston, Inc., Boston, MA, 1983, DOI 10.1007/978-1-47571382-4. MR739850

[Ko12] Bertram Kostant, The cascade of orthogonal roots and the coadjoint structure of the nilradical of a Borel subgroup of a semisimple Lie group (English, with English and Russian summaries), Mosc. Math. J. 12 (2012), no. 3, 605-620, 669, DOI 10.17323/1609-4514-2012-12-3-605-620. MR3024825

[Mu93] B. Mühlherr, Coxeter groups in Coxeter groups, Finite geometry and combinatorics (Deinze, 1992), London Math. Soc. Lecture Note Ser., vol. 191, Cambridge Univ. Press, Cambridge, 1993, pp. 277-287, DOI 10.1017/CBO9780511526336.027. MR 1256283

[Pa94] Dmitrii I. Panyushev, Complexity and nilpotent orbits, Manuscripta Math. 83 (1994), no. 3-4, 223-237, DOI 10.1007/BF02567611. MR1277527

[Pa99] Dmitri I. Panyushev, On spherical nilpotent orbits and beyond (English, with English and French summaries), Ann. Inst. Fourier (Grenoble) 49 (1999), no. 5, 1453-1476. MR 1723823

[RS90] R. W. Richardson and T. A. Springer, The Bruhat order on symmetric varieties, Geom. Dedicata 35 (1990), no. 1-3, 389-436, DOI 10.1007/BF00147354. MR.1066573

[RS92] R. W. Richardson and T. A. Springer, Combinatorics and geometry of K-orbits on the flag manifold, Linear algebraic groups and their representations (Los Angeles, CA, 1992), Contemp. Math., vol. 153, Amer. Math. Soc., Providence, RI, 1993, pp. 109-142, DOI 10.1090/conm/153/01309. MR1247501

[Ro09] Brian David Rothbach, Borel orbits of X2 = 0 in gl(n), ProQuest LLC, Ann Arbor, MI, 2009. Thesis (Ph.D.)-University of California, Berkeley. MR2714006

Université de Lorraine, CNRS, Institut Élie Cartan de Lorraine, UMR 7502, VandoeuVRE-LÈS-NANCY F-54506, FRANCE

Email address: pierre-emmanuel.chaput@univ-lorraine.fr

Université de Lorraine, CNRS, Institut Élie Cartan de Lorraine, UMR 7502, VAndoeuVRE-LÈS-NANCY F-54506, FRANCE

Email address: lucas.fresse@univ-lorraine.fr

Institut Denis Poisson, CNRS UMR 7350, Faculté des Sciences et Techniques, Université de Tours, Parc de Grandmont, 37200 Tours, France

Email address: thomas.gobet@lmpt.univ-tours.fr 